\title{
Fundamental InVESTigations into Single Molecule Surface Enhanced Raman SPECTROSCOPY
}

\author{
BY \\ Camille G. Artur
}

\begin{abstract}
A thesis
submitted to Victoria University of Wellington

in fulfilment of the requirements for the degree of

Doctor of Philosophy
\end{abstract}

Victoria University of Wellington

2014 

To Emilia, mon filou 



\section{Abstract}

After the first claim of single molecule (SM) detection by surface enhanced Raman spectroscopy (SERS) was published in 1997 and years of debate and maturing, SM-SERS can now be considered as an established subfield of SERS. Besides the obvious promising advances in analytical spectroscopy that SM-SERS enables, some more fundamental studies are now also accessible. The main focus of this thesis is to understand certain aspects and tackle some outstanding issues in SM-SERS, both in methods and applications.

In the first part of this thesis, we focus on the application of SM-SERS to the study of the homogeneous broadening of molecular vibrations. We show that the homogeneous linewidth of the dye Nile blue as measured on single molecule SERS spectra is much smaller than the inhomogeneous broadening obtained from the average signal. Individual molecules having the central Raman frequency occurring at slightly different positions is therefore the main cause of the inhomogeneous broadening in this system. Furthermore, we show that the homogeneous broadening of the mode of single molecules exhibits a strong temperature dependence from $80 \mathrm{~K}$ to $300 \mathrm{~K}$. This is suggestive of the vibrational energy exchange model which explicitly relates the temperature dependence of the linewidth of a vibrational mode to its interaction with other modes of the molecule or its environment. The average signal does not show this temperature dependence, this property is indeed washed out by ensemble averaging and its unravelling is made possible by SM-SERS. This study is the first example of direct measurement and study of the homogeneous broadening of a Raman peak.

In the second part of this work, we focus on a particular method 
to prove single molecule sensitivity and demonstrate the single molecule detection of the iconic $\mathrm{C}_{60}$ by SM-SERS using its peculiar spectral properties regarding isotopic substitution. A change in one unit mass in one of the carbon atoms is readily observed as a detectable frequency shift in the $A_{g}(2)$ mode on the Raman spectrum of one $\mathrm{C}_{60}$. This remarkable result is a direct consequence of the high symmetry of the molecule and is only accessible experimentally by measuring individual molecules. We perform SM-SERS detection of a isotopically enriched $\mathrm{C}_{60}$ and show how the distribution of frequencies for the $A_{g}(2)$ mode reflects the isotopic spread of the sample. Density Functional Theory (DFT) calculations support the experimental results. This provides the first ever evidence of single molecule detection of $\mathrm{C}_{60}$ via SERS.

Finally, we focus on the photostability of dyes excited resonantly in SERS conditions. Photobleaching of the molecule is an issue when doing SERS (and SM-SERS) at resonance. Nile blue is deposited on a highly ordered gold nanolithographic substrate and the time dependence of the SERS signal is monitored. Using a simple two-level system model to describe the mechanisms of photobleaching and express the photobleaching rate, we analyse the SERS intensity decay at different powers. This study is the first to be dedicated to the photobleaching decay rates of molecules on metallic surfaces and to highlight that the decay dynamics contains rates spanning four orders of magnitude. This work can potentially reveal information on the distribution of SERS enhancement factors on the surface. 


\section{Acknowledgements}

Being a PhD student in the Raman group at Victoria University of Wellington was a rich experience, academically as well as humanly. Many people contributed to the success of the work presented in this thesis and I will always be in their debt.

First and foremost, I would like to thank my supervisors Pablo Etchegoin and Eric Le Ru who have shown that you can be brilliant, passionate and determined but nevertheless approachable, available and patient. Their support has been very encouraging throughout the years I have spent working on my PhD. The passing of Pablo in April 2013 has caused immense pain and grief in the Raman group and his profound kindness and magical experimental touch will never be forgotten. I would also like to thank Joe Trodahl for his invaluable comments and solid-state perspective.

I would also like to thank my colleagues within the Raman group: Stefan Andreas Meyer, Baptiste Auguié, Walter Somerville, Ben Wiley-van Eerd, Brendan Darby, Peter Hauer, Chris Galloway, Harry Warring, Olly Pantoja, Shrividya Ravi, Lina Jaurigue, Rowan Miller, Marina Craig, Antoine Reigue and Matthias Meyer.

I am grateful to Petrik Galvosas for his support and friendship.

I am also thankful to various SCPS and Faculty staff, especially Natalie Plank, Natasha Evans, Dan Thompson, Eva Anton, the workshop guys, Patricia Stein.

I would like to offer special thanks to Stefan "Andreas" Hertel for his support and his German enthusiasm and to thank Emilia for the countless sleepless nights while writing this thesis and for the promise of many playful days once it is finished. 


\section{Contents}

Abstract $\quad$ i

Acknowledgements

1 Introduction 1

1.1 Optical spectroscopy of molecules . . . . . . . . . . . . 1

1.1.1 Energy levels of molecules . . . . . . . . . . . . 1

1.1.2 Transitions between molecular states . . . . . . 2

1.2 The Raman effect . . . . . . . . . . . . . . . . 6

1.2.1 Terminology in Raman spectroscopy . . . . . . 6

1.2.2 Classical description of Raman scattering . . . . . 7

1.2.3 Resonant Raman scattering . . . . . . . . . . 11

1.2.4 Raman cross-sections . . . . . . . . . . . . . 11

1.3 Surface Enhanced Raman Scattering (SERS) . . . . . . . . . . . . . . . . . 13

1.3.1 Local field enhancement: enhancement of the excitation .................. . . 14

1.3.2 Radiation enhancement . . . . . . . . . . 15

1.3.3 The $|\vec{E}|^{4}$-approximation to SERS enhancement . 15

1.3.4 Influence of the plasmon resonances on the SERS enhancement . . . . . . . . . 16

1.3.5 Current status of SERS . . . . . . . . 18

1.4 Single-molecule Surface Enhanced Raman Scattering (SMSERS) . . . . . . . . . . . . . . . . . . 19

1.4.1 Early evidence for single molecule detection . . . 20

1.4.2 Bi-analyte technique . . . . . . . . . 21 
2 Experimental methods: instrumentation and sample preparation

2.1 Acquiring a Raman spectrum: the Raman setup . . . . 25

2.2 Cryostats . . . . . . . . . . . . . . . 27

2.3 SERS substrates . . . . . . . . . . . . . . . 29

2.3.1 Silver colloids as SERS substrates . . . . . . 30

2.3.2 Preparation of planar SERS substrates: the dried colloids approach . . . . . . . . . . . 35

2.4 SERS active dyes under study . . . . . . . . . . 38

3 Data analysis $\quad 41$

3.1 Non linear least-square fits of the Raman peaks to pseudoVoigt profiles. . . . . . . . . . . . . . . . . . . 42

3.2 Linear fit of a spectrum to a reference . . . . . . . . 45

3.3 Improvement of the linear fits . . . . . . . . . . . 48

4 Measurement of the homogeneous broadening of spontaneous resonant Raman lines via SM-SERS 49

4.1 Introduction . . . . . . . . . . . . . . . . . . 49

4.2 Homogeneous and inhomogeneous broadening in Raman spectroscopy . . . . . . . . . . . . . 50

4.2.1 Homogeneous broadening of a molecular vibration 50

4.2.2 Instrumental broadening . . . . . . . . . . . 57

4.2.3 Mechanisms of inhomogeneous broadening . . . . 59

4.2.4 Anharmonic coupling of phonons and Vibrational Energy Exchange Model (VEE) . . . . . . . . . . 59

4.3 Temperature dependence of the homogeneous broadening of the $590 \mathrm{~cm}^{-1}$ mode of Nile blue . . . . . . . . . . 65

4.3.1 Immediate context of the study . . . . . . . . 65

4.3.2 Experiments . . . . . . . . . . . . 66

4.3.3 Results and discussion . . . . . . . . . . . 67 
4.3.4 Application to the temperature dependence of the homogeneous broadening of the $2230 \mathrm{~cm}^{-1}$ mode of rhodamine $800 \ldots \ldots \ldots . \ldots . \ldots 73$

4.4 Conclusion . . . . . . . . . . . . . . . . 76

5 Single-molecule Surface Enhanced Raman Spectroscopy of $\mathbf{C}_{60}$

5.1 Introduction . . . . . . . . . . . . . . 79

5.2 Structure of $\mathrm{C}_{60}$, icosahedral point group $\mathrm{I}_{h}$ and Raman active modes . . . . . . . . . . . . . . . . . . . . 82

5.2.1 Structure of the $\mathrm{C}_{60}$ molecule . . . . . . . . 82

5.2.2 Symmetry and normal modes of the $\mathrm{C}_{60}$ molecule 83

5.3 Direct isotopic effect on the $\mathbf{A}_{\mathbf{g}}(2)$ mode frequency $\ldots .87$

5.4 Preliminary experiments . . . . . . . . . . . 90

5.4.1 Experimental determination of the differential Raman cross-section of the $\mathrm{Ag}(2)$ mode at $633 \mathrm{~nm}$. 91

5.4.2 DFT calculation of the Raman spectrum of $\mathrm{C}_{60}$ and determination of the differential Raman crosssection of the $A_{g}(2)$ mode . . . . . . . . .

5.4.3 Resolution of the isotopically induced frequency shifts on $\mathrm{C}_{60}$ at $9 \mathrm{~K} \ldots \ldots . . \ldots 99$

5.5 SERS and single-molecule SERS detection of $\mathrm{C}_{60} \ldots \ldots$

5.5.1 SERS experiments on natural $\mathrm{C}_{60} \ldots \ldots . . . .103$

5.5.2 Experiments on $\mathrm{C}_{60}$ commercial isotopologues . . 106

5.5.3 Enhancement factors . . . . . . . . . . . . . 112

5.6 Conclusion . . . . . . . . . . . . . . 117

6 Photostability of dyes under SERS conditions 119

6.1 Background and motivation . . . . . . . . . . 119

6.2 Photobleaching rate and modified photobleaching rate . . 122

6.2.1 A simple model for photobleaching . . . . . . . 122

6.2.2 Modified photobleaching rate in the presence of the metallic surface . . . . . . . . . . . . 125

6.3 Experimental methods . . . . . . . . . . . . . . 128

6.3.1 The SERS substrate: gold nanoparticle arrays . . 128 
6.3.2 Sample preparation . . . . . . . . . . . 130

6.3.3 Acquiring Raman and SERS spectra with the $1 \mathrm{MHz}$ standard mode of the CCD detector . . . . . . . . 130

6.3.4 Optical density filters comparison . . . . . . . . 134

6.3.5 Background corrections . . . . . . . . . . . 135

6.4 Preliminary experiments and results . . . . . . . . . 136

6.5 Conclusions and future work . . . . . . . . . . . . . . 142

Conclusion and outlook

Bibliography 


\section{Chapter 1}

\section{Introduction}

This chapter gives some theoretical background of Raman and surface enhanced Raman scattering (SERS) to support the work which is presented in this thesis. The treatment of the Raman effect and SERS can be extended to any level of complexity; we will however limit here the complexity of the description to the level needed to understand the issues which are raised in the following chapters.

This chapter also aims to set this thesis into the current context of single molecule Surface Enhanced Raman Spectroscopy and giving the motivation and goals I had when I started my PhD at Victoria University within the Raman group.

For detailed and thorough treatments of Raman and SERS, interested readers are invited to consult the text books given in Refs. [1,2].

\subsection{Optical spectroscopy of molecules}

\subsubsection{Energy levels of molecules}

In molecules, the interaction with light is primarily determined by the energy levels of their degrees of freedom: electronic energy levels and atomic motions (nuclei) energy levels. The Jablonski diagram is a conventional way to represent these energy levels in molecular spectroscopy (see Fig. 1.1). The energies of the molecular states are given as a function of the normal mode coordinate and the minima in energy correspond to 
the equilibrium positions of the atoms. The electronic energy levels are the energies of the electronic states of the unperturbed molecule. The vibronic states are sub-states of the electronic states where the electron molecular orbitals are now perturbed by internal vibrations of the nuclei around their equilibrium positions. There are also the rovibronic states which are sub-states of the vibronic states arising from the quantization of the molecular rotation. In the context of this thesis, we will not take into account the subset of rotational states as their energies are too small to be resolved by our spectrometer.

\subsubsection{Transitions between molecular states}

Transitions between molecular states can be induced by various interactions; we can classify molecular transitions into two groups:

- Radiative: or dipole-allowed transitions, they involve the absorption or emission of a photon.

- Non-radiative: transitions between states which do not involve a photon. They can be induced by interactions between vibrations within the molecule (Intramolecular Vibrational Relaxation (IVR)) or interaction with the environment. "Heating" or thermal activation can trigger non-radiative transitions to higher vibrational levels by an amount $\approx k_{\mathrm{B}} T$.

\section{Optical absorption}

A photon of energy $E$ can excite a molecule from a level $E_{1}$ to a level $E_{2}$ with $E_{2}>E_{1}$ and $E=E_{2}-E_{1}$; the photon is annihilated in the process. The photon can only couple to dipole-allowed transitions. In the case of electronic absorption, an electron is excited to a higher energy electronic state and in the case of infrared (IR) absorption, the molecule is excited to a higher energy rotational/vibrational state. 


\section{Energy}

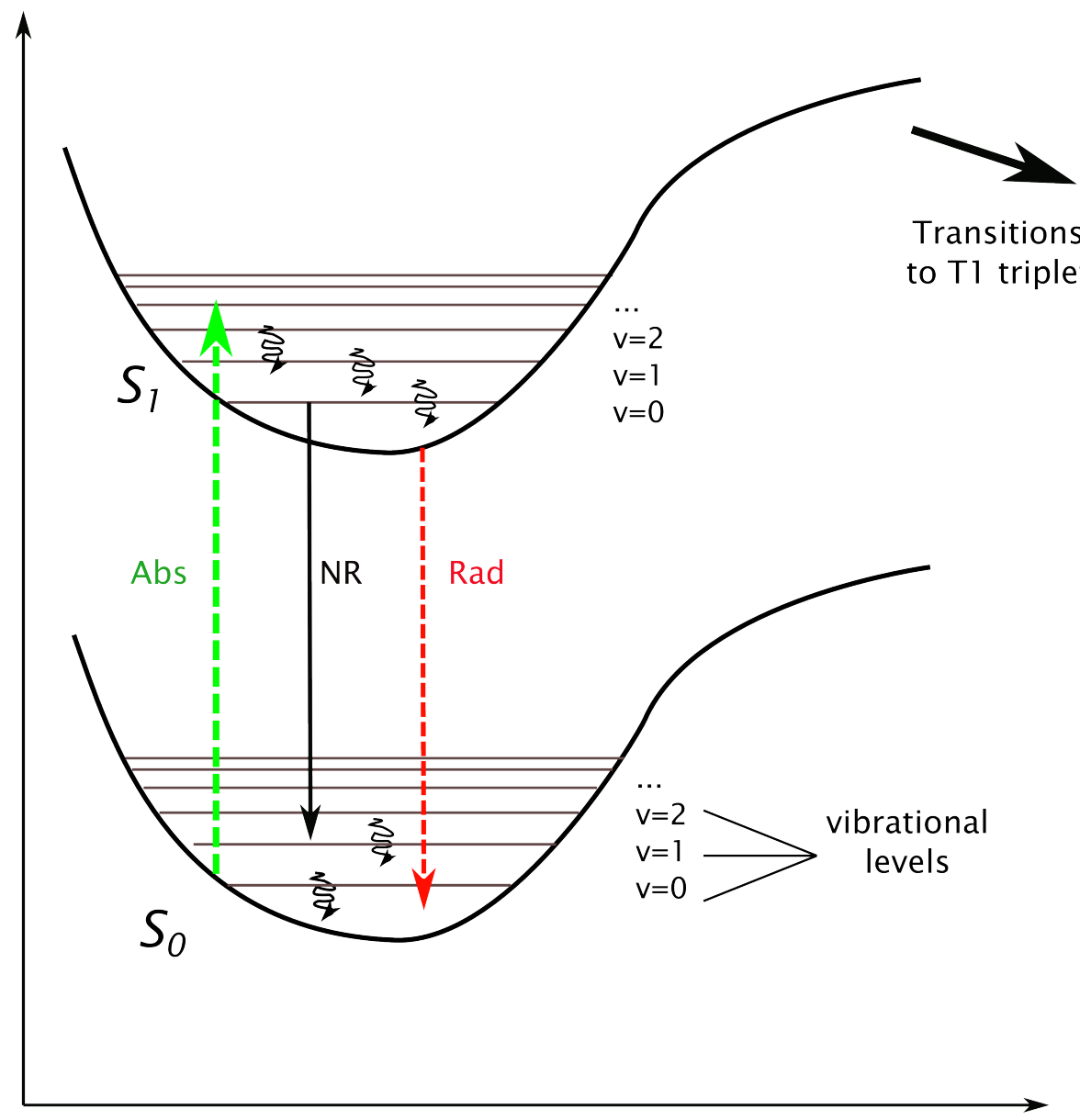

Normal mode coordinate

Figure 1.1: Jablonski diagram of the energy levels of a molecule. The electronic levels $S_{0}$ and $S_{1}$ are in bold and the vibrational levels $v$ are thin lines. Dipole-allowed transitions, absorption in green and spontaneous/stimulated emission in red, are represented by dashed arrows. The black arrow represents the non-radiative processes from $S_{1}$ to $S_{0}$.

\section{Radiative emission and fluorescence}

A molecule in an excited state relaxes from the level $E_{2}$ to the level $E_{1}$ with $E_{2}>E_{1}$ while emitting a photon. Even though radiative emission can occur between two vibrational states, in the cases of interest in this thesis, radiative emission occurs between two electronic states of the molecule. Two processes are possible: 
- Stimulated emission: in the presence of incident photons, the interaction of the excited molecule with the incident photons will stimulate the emission of a photon that is identical to the incident ones. This process occurs at the same rate as the absorption process.

- Spontaneous emission: in the absence of any incident photon, the excited molecule can interact with the vacuum state of the electromagnetic field and this interaction results in the emission of a photon. This is a stochastic process described by a probability per unit time; for the dyes used for the work presented in this thesis, this probability is of the order of $10^{7}-10^{9} \mathrm{~s}^{-1}$.

The two-step process of absorption of a photon followed by emission is called luminescence, the most common case being that of fluorescence:

* An incident laser beam of energy $E_{\text {inc }}$ excites an electron from $S_{0}$ to a vibronic state of $S_{1}$.

* The electron quickly (picosecond [3]) relaxes down to the vibrational ground state of $S_{1}$ through IVR or solvent interaction.

* From there, several transitions are possible but if spontaneous emission of a photon of energy $E_{\mathrm{S}}<E_{\text {inc }}$ occurs associated with the electron relaxation down to $S_{0}$, the overall process is called fluorescence.

Fluorescence is therefore a two-step process and happens on the same time-scale as spontaneous emission. The energies of the fluorescent photons vary and form the fluorescence spectrum.

\section{Light scattering processes}

The optical processes discussed previously involve the absorption or emission of a single photon. Another important family of processes involves the simultaneous (instantaneous) absorption and emission of a photon. Such processes are called scattering processes and can be classified into two groups: 

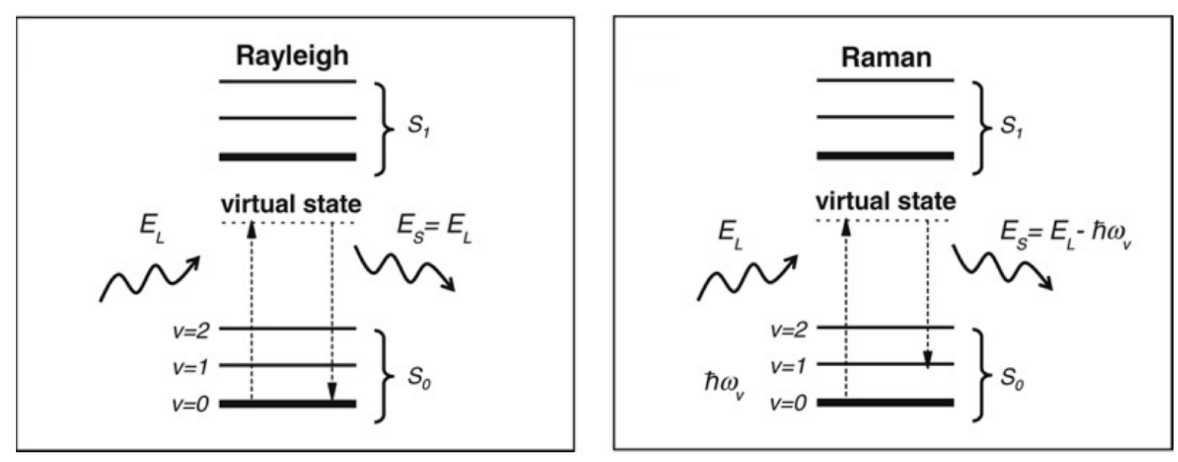

Figure 1.2: Simplified Jablonski diagrams illustrating the Rayleigh (left) and Raman (right) scattering processes. From a quantum mechanical point of view, the scattering can be viewed as two simultaneous processes; absorption of a photon through a transition to a virtual state and emission of a photon through recombination to $S_{0}$.

- Elastic scattering: incident and scattered photons have the same energy (but different polarization and direction). For molecules, hence in the case where the size of the object is much smaller than the wavelength (visible-IR), this process is referred to as Rayleigh scattering.

- Inelastic scattering: the scattered photon is at a different energy $E_{S}$ from that of the incident photon $E_{L}$. The energy difference corresponds to a transition between two states in the molecule. In the case of Raman scattering, transitions between vibrational/rotational states are involved.

Rayleigh and Raman scattering are illustrated in Fig. 1.2. The important difference between Raman scattering and fluorescence is that the Raman process is instantaneous while fluorescence is a two-step process which require absorption of a photon then spontaneous emission. Raman scattering can occur without direct absorption of a photon, therefore when no electronic transition exists in the molecule at the incident wavelength. However scattering processes (which can be described in a quantum treatment of the interaction between light and matter as second order perturbations) are intrinsically weak phenomena when compared to other optical processes like absorption or fluorescence. 


\subsection{The Raman effect}

The Raman effect was named after Sir Chandrasekhara Venkata Raman who discovered it and published it in the journal Nature in 1928 [4] (Nobel prize in physics awarded in 1930). Sunlight focused through a telescope as excitation and crossed complementary monochromatic filters were used for the experiment; a small amount of light had changed frequency and could pass the second filter. The experiments were quite challenging as the Raman scattered light was very weak; therefore the scatterers used were highly concentrated liquids. Progress in Raman spectroscopy as a technique was stalled until the invention of lasers in the 1960s as powerful monochromatic sources.

Raman spectroscopy is non-destructive, can be carried out in air, or water (which does not exhibit any strong background signal in Raman) and the sample preparation is usually quite straightforward. Furthermore, Raman spectroscopy can be performed at any incident wavelength as no absorption of the incident photons by the sample is required. A Raman spectrum is a unique fingerprint of a molecule which can be used for analytical purposes in many cases and combinations. It is much more specific than other commonly used optical techniques like fluorescence spectroscopy.

The main drawback however, is that the Raman effect is intrinsically weak, much weaker than fluorescence.

\subsubsection{Terminology in Raman spectroscopy}

Before giving a description of the Raman effect, let us define here some terms and conventions specific to Raman spectroscopy:

- If the scattered photon has less energy than the incident photon $E_{S}<E_{L}$, then the molecule is excited to a higher vibrational energy level. This is called a Stokes process and typically corresponds to the excitation of the molecule from the ground vibrational state $v=0$ to the first vibrational excited state $v=1$ by creating a vibrational mode of energy $\hbar \omega_{v}=E_{L}-E_{S}$. 
- If, on the contrary, the scattered photon has more energy than the incident photon $E_{S}>E_{L}$, then the molecule has relaxed from an excited vibrational state $v=1$ to its ground state $v=0$. this is called anti-Stokes process. In order for the process to occur, the molecule should already be in an excited vibrational state. This may only happen through thermal activation, therefore the antiStokes signal depends on the temperature $T$ through a Boltzmann factor. Hence the anti-Stokes side of a Raman spectrum is much weaker than the Stokes side.

- The energy lost in the scattering event is called the Raman shift and is commonly expressed in wavenumber units $\left[\mathrm{cm}^{-1}\right] ; \Delta E_{R}=$ $E_{L}-E_{S}$. The Raman shift is hence positive for Stokes and negative for anti-Stokes.

- The Raman spectrum is commonly shown as Raman intensity as a function of Raman shift as shown on Fig.1.3. Peaks in the spectrum correspond to Raman-active vibrational modes of the molecule. This spectrum is a unique fingerprint of a molecule.

\subsubsection{Classical description of Raman scattering}

This section presents a classical and phenomenological description of the Raman effect. Raman scattering should in principle be described by a quantum theory, however this approach is not straightforward and is not a necessary tool to the comprehension of the work presented in this thesis. A complete semi-classical derivation (the molecule is treated quantum mechanically but the electromagnetic field is described classically) of the Raman effect can be found in Ref. [2].

\section{Classical approach to optical scattering}

The classical approach to scattering makes use of the fact that the emission of light from a small source (like an emitting molecule) can be described in terms of multipolar components, the dominant one being the electric dipole. 


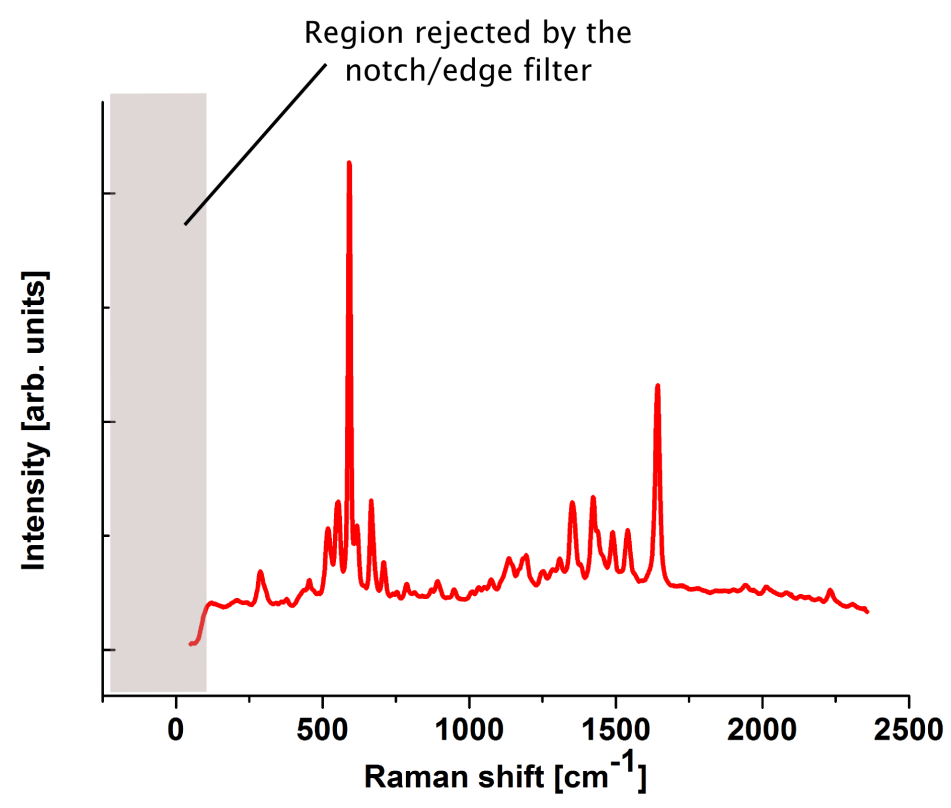

Figure 1.3: A typical Raman spectrum (in fact SERS spectrum of Nile blue at $633 \mathrm{~nm}$ ). The Raman intensity is a function of the Raman shift. The zero hence corresponds to unshifted photons i.e. light at the laser wavelength. The central line has to be rejected lest it destroys the very sensitive photo-detectors used in a Raman spectroscopy experiment. Notch or edge filters are used for that purpose.

An electric dipole $\vec{p}(t)$ oscillating at the frequency $\omega$ radiates (emits) photons at energy $\hbar \omega$. Using complex notation, $\overrightarrow{\mathrm{p}}(t)$ is:

$$
\overrightarrow{\mathrm{p}}(t)=\Re\left(\underline{\overrightarrow{\mathrm{p}}} \times e^{-i \omega t}\right)
$$

The scattered radiation can be described as the radiation emitted by a dipole. To describe how the incident beam gives rise to an oscillating dipole, the notion of polarizability is introduced. The molecule is characterized, in absence of perturbation, by the position of the nuclei and its orbitals. In the presence of an external perturbation, the electronic orbitals change and the charge redistribution constitutes an induced dipole.

The incident electric field $\underline{\vec{E}}(\omega)$ oscillating at the angular frequency $\omega$ induces a dipole $\underline{\mathrm{p}}_{L}(\omega)$ such as: 


$$
\underline{\vec{p}}_{L}\left(\omega_{L}\right)=\hat{\alpha}_{L} \underline{\overrightarrow{\mathrm{E}}}\left(\omega_{L}\right)
$$

where $\hat{\alpha}_{L}$ is the linear polarizability tensor or Rayleigh polarizability tensor; it fully characterizes the linear response of a molecule to an incident electric field. The polarizability provides a measure of the "responsiveness" of the electrons in a molecule to the presence of an external electric field. The dipole radiates at the same angular frequency as the incident field; this is the classical description of Rayleigh scattering.

\section{The Raman tensor}

In the description of $\hat{\alpha}_{L}$ above, it is implicit that the molecule is in its ground-state geometry, i.e. minimum of its electronic energy and the atoms are static. However nuclei can vibrate around their equilibrium positions and these vibrations can be seen as small perturbations to the electronic structure, hence a small perturbation to $\hat{\alpha}_{L}$. This small perturbation is at the origin of Raman scattering.

A molecule with $N$ atoms has $3 N$ internal degrees of freedom. Vibrational analysis consists in finding the $3 N$ normal modes of the molecule (eigenvectors of the Hessian matrix or force constant matrix, which corresponds to the secondary derivatives of the potential energy of the molecule with respect to atomic displacements). In Section 5.4.2, vibrational analysis is presented and we will see how this is achieved numerically for the molecule $\mathrm{C}_{60}$. Once the normal modes and the normal mode coordinates are known, we can express the induced changes in the linear polarizability $\hat{\alpha}_{L}$ when the molecule is deformed by a normal mode vibration.

We define the Raman tensor of the normal mode $k$ by:

$$
\hat{R}_{k}\left(\omega_{L}\right)=\left(\frac{\partial \hat{\alpha}_{L}\left(\omega_{L}\right)}{\partial Q_{k}}\right)_{Q_{k}=0}
$$

where $Q_{k}$ is the mass-weighted normal mode coordinate. From there, we can see that, if $\hat{\alpha}_{L}\left(\omega_{L}\right)$ does not depend on $Q_{k}$ (very common for highly 
symmetric molecules), then the Raman tensor for the mode $k$ is zero and the mode is Raman-inactive.

\section{The classical Raman dipole}

We may phenomenologically generalize the classical description of Rayleigh scattering given previously to the case of inelastic scattering, in particular Raman scattering. In the case of Stokes scattering from a vibrational mode of energy $\hbar \omega_{k}$, the scattered radiation is at a frequency $\omega_{R}=\omega_{L}-\omega_{k}$ different from the incident frequency $\omega_{L}$. The induced Raman dipole must therefore oscillate at the radiated frequency $\omega_{R}$ and is denoted $\underline{\vec{p}}_{R}\left(\omega_{R}\right)$.

We can define, by analogy with Rayleigh scattering, the Raman polarizability tensor $\hat{\alpha}\left(\omega_{L}, \omega_{k}\right)$ by:

$$
\underline{\vec{p}}_{R}\left(\omega_{R}\right)=\hat{\alpha}\left(\omega_{L}, \omega_{k}\right) \cdot \underline{\vec{E}}\left(\omega_{L}\right)
$$

$\hat{\alpha}\left(\omega_{L}, \omega_{k}\right)$ is a proportionality factor which is fully phenomenological and cannot be considered as a linear response function as was the case for the linear polarizability tensor $\hat{\alpha}_{L}$.

The phenomenological Raman polarizability $\hat{\alpha}\left(\omega_{L}, \omega_{k}\right)$ for the normal mode $k$ is proportional to the Raman tensor $\hat{R}_{k}\left(\omega_{L}\right)$ [1]:

$$
\hat{\alpha}\left(\omega_{L}, \omega_{k}\right)=\frac{Q_{k}^{0}}{2} \hat{R}_{k}\left(\omega_{L}\right)
$$

where $Q_{k}^{0}$ is the amplitude of the vibration $k$ which caused the Raman scattering effect. Hence the perturbation caused by the vibration produces a "modulation" of the linear polarizability resulting in a beating of frequencies between the frequency of the excitation $\omega_{L}$ and the frequency of the vibration $\omega_{k}$; this beating phenomenon results in inelastic scattering, i.e. in Raman scattering. 


\subsubsection{Resonant Raman scattering}

As seen earlier, Raman scattering can occur without direct absorption of a photon, i.e. when no electronic transition exists in the molecule at the incident wavelength (which is not the case for fluorescence). Nevertheless, when the incident angular frequency $\omega_{L}$ is close to a real electronic transition frequency of the molecule, a resonant response occurs and can account for at least four orders of magnitude changes in the Raman intensity.

A semi-classical treatment of Raman scattering, where the molecule is described quantum mechanically but not the electromagnetic field, is necessary to provide a qualitative explanation for resonant Raman scattering. From the quantum treatment of the scattering process [2], a given component of the Raman tensor can be shown to be a sum over all possible vibronic states of the molecule of transition probabilities between an initial state and a final state (computed by perturbation theory). One feature of these expressions is the energy denominators; when they are small, i.e. when $\omega_{L}$ is close to a real electronic transition frequency, then resonance is expected.

Resonant effects and even pre-resonance effects can have a large influence on the Raman cross-section of typical SERS probes.

\subsubsection{Raman cross-sections}

The absolute differential Raman cross-section $\frac{\mathrm{d} \sigma_{R}}{\mathrm{~d} \Omega}$ is (by definition [1]) derived from:

$$
\frac{\mathrm{d} P_{R}}{\mathrm{~d} \Omega}\left(90^{\circ}\right)=\frac{\mathrm{d} \sigma_{R}}{\mathrm{~d} \Omega} S_{\mathrm{inc}}
$$

It relates the molecular-orientation-averaged differential Stokes Raman scattered power at $\omega_{R}=\omega_{L}-\omega_{v}$ observed in the $90^{\circ}$-configuration to the incident power density $S_{\text {inc }}$. Differential here means per unit solid angle $d \Omega$. It characterizes the scattering efficiency of a certain vibrational mode of energy $\hbar \omega_{v}$ in a certain direction of observation with respect to the incident field. It is expressed in $\mathrm{cm}^{2} / \mathrm{sr}$ units. 
$\frac{\mathrm{d} \sigma_{R}}{\mathrm{~d} \Omega}$ for a particular mode depends on the incident wavelength and on the refractive index of the environment. We can show that the absolute differential Raman cross-section and more generally all optical scattering cross-sections have a dependence in $\omega_{R}^{4}$; this factor in fact comes from the electromagnetic theory of dipole emission.

The direct accurate measurement of the absolute differential Raman cross-section is a complex issue and requires the careful characterization of the whole experimental set-up [5]. Such studies have only been carried out for a handful of compounds ( $\mathrm{N}_{2}$ gas, benzene, cyclohexane [5]) which hence serve as standards. The relative cross-sections of other compounds are then determined by comparing the Raman intensity with the Raman intensity of the standard. As long as the exact same experimental conditions (excitation wavelength, optics, solvent) are used for both the sample and the standard, the absolute differential Raman cross-section of the sample can be determined via the ratio of peak intensities and of concentrations. Throughout this thesis, $\mathrm{N}_{2}$ gas, 2B2MP (2-bromo2-methylpropane) and toluene were used as standard references in the experimental determination of Raman cross-sections at $633 \mathrm{~nm}$ excitation.

Within the context of SERS, one must quantify the cross-sections of molecules that are used as SERS probes, among which the most commonly used are dyes (Nile blue, Rhodamine 6G, Crystal violet, ...). However, the determination of their Raman cross-sections at resonance is tricky due to the overwhelming intensity of the fluorescence background. An experimental method to overcome this particular issue has been recently developed by our group and is presented in Refs. [6, 7].

Table 1.1 provides values for the absolute differential Raman crosssections of the fingerprint Raman modes of the three standards we use routinely in our group as references and the dye Nile blue A in water (in resonance) at $633 \mathrm{~nm}$. Note the dramatic difference in the values of $\frac{\mathrm{d} \sigma_{R}}{\mathrm{~d} \Omega}$ between the cross-sections off-resonance for typical organic liquids (such as 2B2MP and toluene) or a very small molecule like $\mathrm{N}_{2}$ and the cross-section of Nile blue measured at resonance. 


\begin{tabular}{|c|c|c|}
\hline & Mode frequency $\left[\mathrm{cm}^{-1}\right]$ & $\frac{\mathrm{d} \sigma_{R}}{\mathrm{~d} \Omega}\left[\mathrm{cm}^{2} / \mathrm{sr}\right]$ \\
\hline $\mathrm{N}_{2}$ gas & 2230 & $2.1 \times 10^{-31}$ \\
\hline Toluene & 1002 & $3.5 \times 10^{-30}$ \\
\hline 2B2MP & 516 & $5.6 \times 10^{-30}$ \\
\hline Nile blue A (water) $^{a}$ & 590 & $1.7 \times 10^{-24}$ \\
\hline
\end{tabular}

Table 1.1: Absolute differential Raman cross-sections at $633 \mathrm{~nm}$ for the fingerprint modes of the three standards ${ }^{b}$ used in this thesis and the dye Nile blue in water.

${ }^{a}$ From Ref. [7]

${ }^{b}$ From Ref. [1]

\subsection{Surface Enhanced Raman Scattering (SERS)}

The SERS effect is the amplification of the Raman signal of molecules on or close to (few $\mathrm{nm}$ ) a metallic surface through electromagnetic interaction of light with the metal; these interactions produce a large enhancement of the incident field through the excitation of localized surface plasmon resonances, which are electromagnetic surface modes of metallic systems with dimensions smaller than or of the order of the incident wavelength. The metallic systems - or SERS substrates - can be for example colloidal solutions of metallic nanoparticles, planar substrates onto which colloids have been aggregated, arrays of nanoparticles produced by electron beam lithography, etc.

SERS was discovered in 1974 [8] for pyridine molecules adsorbed onto roughened electrodes and wrongly attributed to an increase in the effective surface area. The effect was correctly interpreted in 1977 by two independent and simultaneous studies $[9,10]$. During the last 40 years, the field has matured and grown enormously and is now a well established spectroscopic technique. It is multi-disciplinary by nature as it encompasses aspects such as electromagnetic theory of plasmon resonances in a metals, colloidal solutions, molecular adsorption, substrate nano-fabrication and applications in analytical chemistry and biology.

The discovery of SERS has addressed the main issue of Raman spec- 
troscopy: the sensitivity. SERS combines the high specificity of Raman spectroscopy with a much higher sensitivity which then becomes comparable to or greater than fluorescence. SERS can, in principle be applied to any analyte, provided that one manages to bring the molecule in close vicinity to a SERS efficient metallic substrate. SERS therefore has many potential applications in analytical chemistry, biochemistry, forensic sciences, trace analysis, analysis of dyes from old artwork, etc. SERS has also immensely benefited from the development of nanosciences and nanotechnologies in terms of SERS substrate design and fabrication and from the development of plasmonics in the last decade or so.

The most widely used SERS metals (i.e. providing the enhancement of the signal) are gold and silver as their electromagnetic resonances are located in the visible or near-infrared region of the light spectrum and as they have low absorption losses in that regime.

The following sections present some key points necessary to the understanding of the electromagnetic origins of the SERS enhancement of the signal. The molecule will be seen as a dipole whose excitation and emission are modified by the presence of the metallic surface/system.

\subsubsection{Local field enhancement: enhancement of the excitation}

When the excitation wavelength $\lambda_{L}$ is close to the electromagnetic resonance of the metallic system, the electric field at the molecule position (on or near to the metal) $\vec{E}_{\text {loc }}$ can be very different, both in magnitude and orientation, from the incident field $\vec{E}_{\text {inc }}$. $\vec{E}_{\text {loc }}$ is called the local field. In the case of SERS, the magnitude of $\vec{E}_{\text {loc }}$ can be much, much larger than the magnitude of $\vec{E}_{\text {inc }}$.

The local field induces a Raman dipole (through the molecular polarizability) $\vec{p}_{R}=\hat{\alpha}_{R} \vec{E}_{\mathrm{loc}}\left(\omega_{L}\right)$ whose magnitude is therefore enhanced by a factor $\left|\vec{E}_{\mathrm{loc}}\left(\omega_{L}\right)\right| /\left|\vec{E}_{\mathrm{inc}}\right|$. If such a dipole radiates in free space (no metal), then the energy (intensity) radiated would be enhanced by a factor: 


$$
M_{\mathrm{loc}}\left(\omega_{L}\right)=\frac{\left|\vec{E}_{\mathrm{loc}}\left(\omega_{L}\right)\right|^{2}}{\left|\vec{E}_{\mathrm{inc}}\right|^{2}}
$$

This factor is called local field intensity enhancement factor and is associated with the enhanced excitation of the Raman dipole, in simple terms, the molecule will be polarized by an enhanced local electric field. For an absorbing molecule (such as a dye) it also characterizes the enhancement of the absorption cross-section, as we will see in Chapter 6 . Electromagnetic calculations predict that $M_{\text {loc }}$ can be as large as $\sim 10^{5}$ at the plasmon resonance.

\subsubsection{Radiation enhancement}

Under SERS conditions, the Raman dipole (the molecule), radiates, not in free space, but in close proximity to the metal. The dipole radiation is strongly affected by the metallic surface, in a similar manner to the dipole excitation. It affects the dipole in two ways:

- The radiation pattern is modified.

- The total power radiated by the dipole is modified compared to that in free space.

We can thus define a radiation enhancement factor $M_{\text {rad }}$. In the case of SERS, for a dipole of given amplitude, more total energy will be extracted from it compared to a dipole of same amplitude in free space.

\subsubsection{The $|\vec{E}|^{4}$-approximation to SERS enhancement}

For one molecule in SERS conditions, the total single molecule SERS electromagnetic enhancement factor (SMEF) can be simply expressed as:

$$
\mathrm{SMEF} \approx M_{\mathrm{loc}}\left(\omega_{L}\right) M_{\mathrm{rad}}\left(\omega_{R}\right)
$$


The problem is that estimating $M_{\mathrm{rad}}$ is a priori a difficult task, whereas, $M_{\text {loc }}$ can be found by solving the electromagnetic problem under specific external conditions. To avoid this issue, it is often assumed (and can be justified using the optical reciprocity theorem [11]) that $M_{\mathrm{rad}} \approx M_{\mathrm{loc}}$. Moreover, in many cases, the Raman shift is also small compared to the incident and scattered energies so that we can consider that $\omega_{R} \approx \omega_{L}$. thus Eq. 1.8 becomes:

$$
\operatorname{SMEF}\left(\omega_{L}\right) \approx \frac{\left|\vec{E}_{\mathrm{loc}}\left(\omega_{L}\right)\right|^{4}}{\left|\vec{E}_{\mathrm{inc}}\right|^{4}}
$$

otherwise known as the $|\vec{E}|^{4}$-approximation.

This approximation is sufficient to know the right order of magnitude of the electromagnetic enhancement of the Raman intensity experienced by a single molecule under SERS conditions, when $\left|\vec{E}_{\mathrm{loc}}\left(\omega_{L}\right)\right|$ at the molecule's position can be evaluated (by an electromagnetic computation for example).

\subsubsection{Influence of the plasmon resonances on the SERS enhancement}

A complete treatment of this issue is given in Ref. [1] and we will here just summarize some key points.

- The localized surface plasmon (LSP) modes of a metallic nanoparticle (or more complex structures) can be excited by an incident wave with the appropriate polarization and frequency. This corresponds to a strong optical response which can be detected in a UV-visible absorption or extinction experiment. Large local field enhancement are obtained at the LSP resonance of the metallic structures. These LSP resonances are, fortunately for SERS, quite broad and generally a large range of incident wavelengths can be used to "activate" the SERS substrate. For examples of resonance spectra of SERS active metallic colloidal solutions used for the work presented in this thesis, please refer to Section 2.3. 


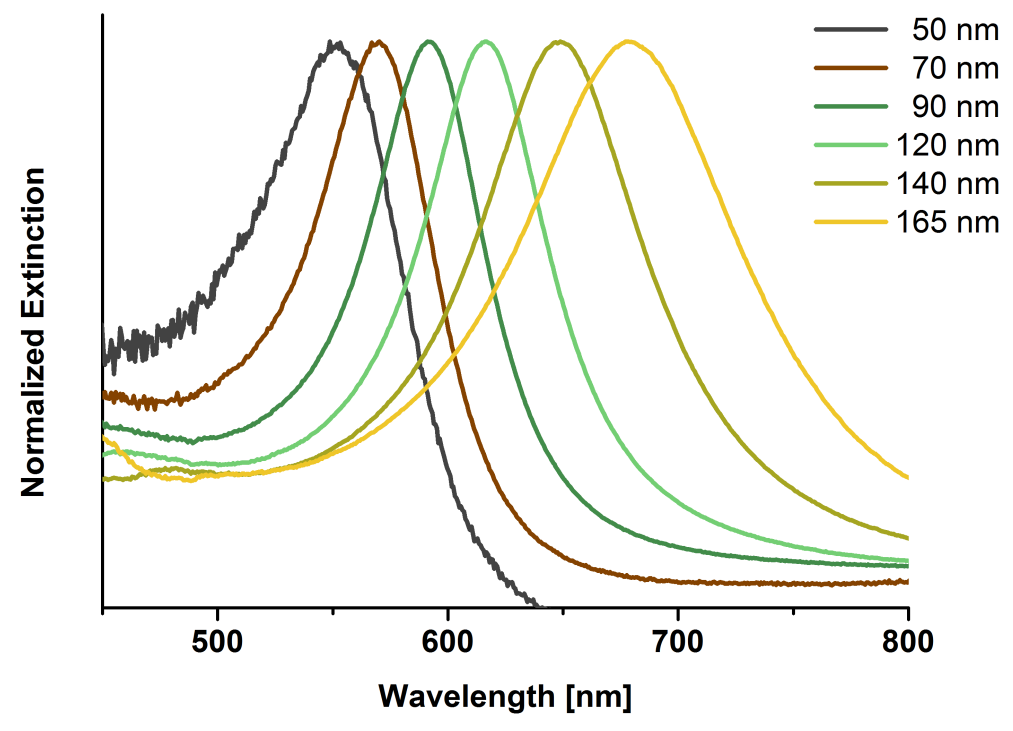

Figure 1.4: Normalized extinction spectra of the arrays of nanoparticles used in the photobleaching studies of Chapter 6. They are arrays of gold nanodiscs whose diameter is given in the legend. It can readily be seen that the position of the LSP shifts towards greater wavelengths when the size of the nanoparticles increases.

- Size effects: LSP resonances tend to redshift when the size of nanoparticles increases. The resonances tend also to broaden. Figure 1.4 shows an example of the shift towards greater wavelengths of the LSP resonances when the diameters of gold nanodiscs increase.

- Shape effects: the aspect ratio of a particle and its curvature (flatter or pointier) dramatically affect its LSP resonances, the magnitude and the localization of the enhancement in the electric field. Usually, the more "pointy" the shape, the larger the enhancement factor. However, the place where the largest EF is achievable on the particle becomes increasingly localized to a very small area.

- Gap effects: plasmon resonances and electromagnetic enhancements also arise from the interaction of two or more objects. This interaction becomes increasingly important at small separation between the particles (small gaps), where large local fields $\left|\vec{E}_{\text {loc }}\right|^{2}$ result in 
some of the largest known values for the SMEF: $\sim 10^{11}$.

\section{Hot spots}

We finish this section by introducing the notion of hot spot, a widely used term in the SERS literature. A hot spot is a point of very large local field intensity enhancement. Hot spots are usually highly localized and can be within a few nanometers of points with little or negligible enhancement. Usually the SERS signal from molecules located at the hot spots of nanoparticles in a SERS substrates will dominate the average SERS signal. For example, in Fig. 1.5, a molecule at the tip of the prolate spheroid in (a) will emit as much as 100 molecules adsorbed on the green area. More drastically, if a colloidal solution was constituted of dimers of spheres as in (b) and used as a SERS substrate with an homogeneous coverage in molecules, the detected SERS signal would be completely dominated by the few molecules located at the gap junctions between particles.

The concept of hot spot localization is crucial in SERS, because of the usually extreme spatial variations of the enhancement on most substrates $[13,14]$.

\subsubsection{Current status of SERS}

Surface enhanced Raman spectroscopy as a technique has progressed over the last 40 years through contradictions, disagreements and heated debates. One of the hottest issues was the origin and nature of the SERS enhancement. Over the years, many of these conflicts have been resolved to some degree and the electromagnetic enhancement factor EF is thought to be by far the main contribution to the enhanced signal. An additional "chemical" enhancement is possible and often referred to antagonistically by some authors as part of the SERS enhancement; it would be a mechanism concerning only certain probes when they are chemically adsorbed onto the metal, these molecules would see their electronic resonance shifted by this adsorption by means of charge-transfer, resulting in some more enhancement of the signal. Its existence is still subject to 

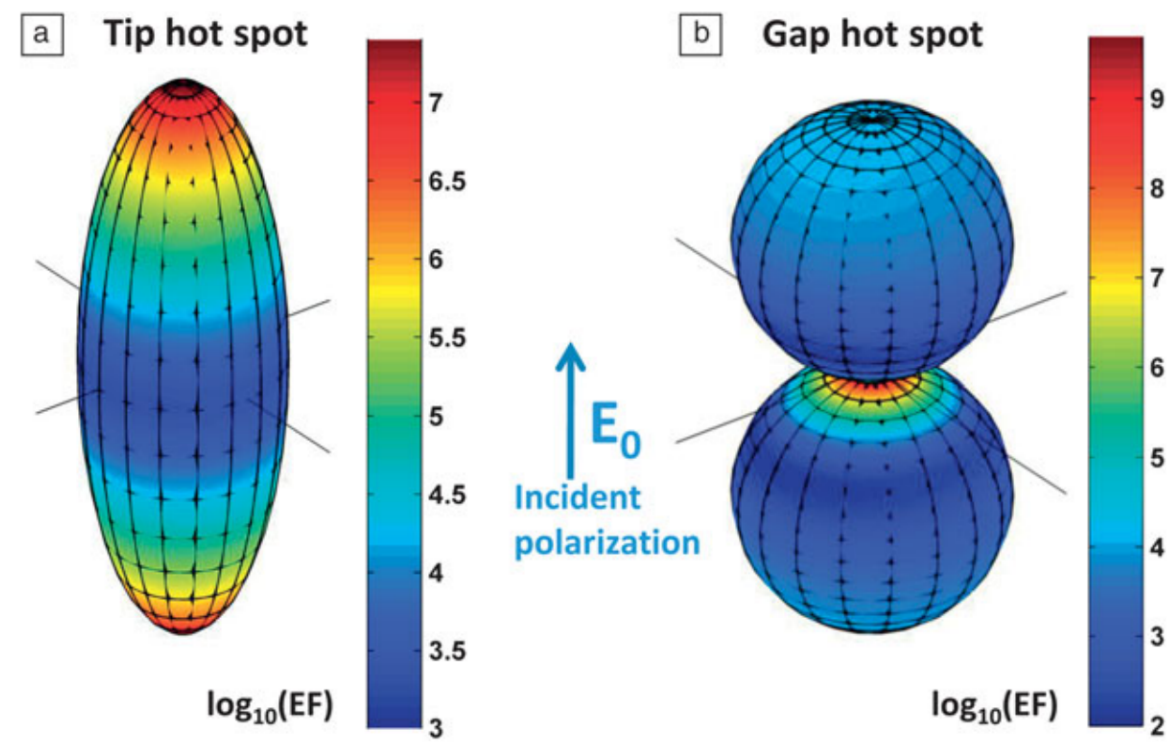

Figure 1.5: Typical electromagnetic hot spots in nanostructures. They are evidenced by computing the distribution on the surface of the SERS enhancement factor $(\mathrm{EF}) M_{\mathrm{loc}}^{2}$. Both calculation were done with an incident field at the LSP resonance of the particles. (a) An elongated Ag spheroid exhibits two tip hot spots where the EF reaches values close to $10^{7}$. (b) More spatially localized hot spots can be obtained in gap hot spots as shown in the gap between two nanospheres with EF of $10^{1} 0$. Adapted from Ref. [12].

controversy [15], the point being that it makes the chemical enhancement a property which is probe-dependent, hence not an intrinsic property of a SERS substrate. Nevertheless, the mechanisms underlying SERS are complex and the story is not yet complete regarding some of the fundamental aspects; the modification of the electronic structure of some molecules via adsorption being in fact a fascinating topic that SERS can help investigate [16].

\subsection{Single-molecule Surface Enhanced Ra- man Scattering (SM-SERS)}

This section is dedicated to arguably one of the most interesting developments in SERS: the realization and demonstration that a single molecule 
can be detected by SERS.

In 1997, two independent reports claimed the observation of single molecule emission under SERS conditions $[17,18]$. The appeal of singlemolecule spectroscopy is huge for two main reasons:

- SM-SERS indeed gives the possibility to push analytical tools to the ultimate resolution limit: the single molecule. Even though fluorescence spectroscopy had already achieved the single molecule detection level, SM-SERS has a unique high spectral specificity, it is applicable to non-resonant probes and infrared excitation is possible. Naturally, the potential applications in biology are very attractive and a considerable amount of SM-SERS work published nowadays is in relation to biologic systems or issues.

- SM-SERS also suggests the possibility of many fundamental applications. It has the potential to allow the understanding of unique single molecule phenomena that are washed out by ensemble averages. It could also lead to a better understanding of SERS itself.

Today, not many researchers in the field of SERS would doubt the reality of SM-SERS detection but the path was not free of controversy and problems; which were emphasized by the lack of reproducibility, lack of understanding of the origins and conditions for SM-SERS (in terms of enhancement factors), lack of control over the hot spot distribution and, last but not least, the confusing early indirect proofs for single molecule detection. And these past issues are today still combined with a general confusion on how to evaluate reliably SERS enhancement factors (first attempt at a standardization of the definitions and evaluation of the EF in 2007 [19]) and more generally some still deep rooted misconceptions regarding SERS [20].

\subsubsection{Early evidence for single molecule detection}

By far, the largest group of evidence for single molecule detection by SERS comes from an "ultra low concentration" approach [21] with the use of metallic colloids as SERS substrates either in liquid form or dried 
onto a planar surface. The analyte concentration was chosen to be very low compared to that of nanoparticles, hence the argument was that, statistically speaking, there could not be many more than one molecule per colloid or one molecule in the scattering volume. However, the use of concentrations in analyte well below $\mathrm{nM}$, of the order of $\mathrm{pM}$, requires particular care in the materials and methods to avoid contamination, wall adsorption, dilution errors, etc. Furthermore, it is quite impossible to estimate the accurate concentration of colloids and their distribution of sizes and shapes from a knowledge of the reactant proportions for the synthesis of the colloids. The presence of a small number of large aggregates could lead to a dramatic underestimation of the analyte:colloid ratio. Finally, at low concentrations, the probability to have a molecule find its way to a hot spot is extremely small, making the statistics of the SM-SERS signal unreliable.

Another argument put forward as evidence was the fact that the SERS signals were exhibiting strong fluctuations. However, such fluctuations are also observed in SERS conditions even with high concentrations of analytes. These fluctuations are more to be attributed to the fluctuations of the SERS enhancement factors within the SERS substrate.

\subsubsection{Bi-analyte technique}

The bi-analyte technique is another approach to the single molecule SERS problem. It was developed by our group in 2006 [21] and addresses the issue of the unreliable statistics.

It consists in measuring the SERS signal from a mixture of two molecules with distinguishable SERS spectrum and preferably comparable cross-sections. If the concentration of the two analytes is such that there are many molecules at hot spots, the SERS signal should in principle be a mixture of the two spectra. The observation of SERS signals of purely one type or the other is clear evidence that it comes from a very small number of molecules.

For example, if a SERS signal originates from, say, 5 molecules, the probability of it being purely of analyte 1 is only $1 / 32$ and this prob- 
ability decreases to 1/1024 for 10 molecules. By carrying out several such experiments at decreasing concentrations, one should in principle observe a transition from a regime where only mixtures of the two spectra are observed to a regime where the majority of observed spectra are "pure". The non-mixed spectra may then be attributed to either single molecules or a few molecules of the same type. Such a study allows one to determine for a given system [SERS substrate + analyte] the preparation conditions and concentrations for which single molecule spectra are likely to be obtained.

The technique has been since then implemented in different variations $[22,23,24,25]$, notably the use of isotopically edited probes as bi-analyte partners $[26,25]$. With isotopic substitution, the change in adsorption properties is expected to be minimal but there can still be measurable differences in the Raman spectra .

In summary, the bi-analyte technique is simple, unambiguous and applicable to a wide variety of SERS substrates. The implementation of this technique has also shown that, in fact, single molecule SERS is quite common even at relatively high concentration, due to the extreme localization of hot spots.

\subsubsection{General motivation and thesis outline}

Nowadays, the field of single molecule SERS is considered as a well established subfield of SERS or laser spectroscopy and the conditions under which SM-SERS can be observed have become increasingly well understood. Besides the analytical possibilities, we can exploit some more "fundamental" applications of the technique:

- SM-SERS can be a tool to further our understanding of the mechanisms of SERS. For example, in Ref. [27], the comparison of SERS over fluorescence ratios in single molecule spectra unveils the relative dominance of the non-radiative enhancement in SERS conditions. Another study was dedicated to charge transfer mechanisms at the single molecule level [28]. Single molecule studies have also 
shown that the measured maximum SM-SERS EF may be artificially affected by photobleaching in some cases [29].

- The detection of non-resonant probes by SM-SERS is in itself a challenging goal. Using the concept of isotopic substitution, to create bi-analyte partners, SM-SERS detection of adenine was demonstrated [30]. Other types of molecules have also been detected [31]. The conditions required for SM-SERS detection of non-resonant molecules present more challenges than for resonant or pre-resonant molecules as contamination issues become problematic and the SERS substrate must also provide increased enhancement. For that reason, the vast majority of SM-SERS studies to date are carried out on resonant molecules.

- SM-SERS is also now a promising tool to investigate indirectly properties of molecules that are accessible at the single molecule level but are hidden in measurements of an ensemble of molecules. As an example, the determination of the resonance excitation spectrum of a single molecule performed in Ref. [16]. Another example is the study of single molecule conductance and/or heating properties $[32,33]$. Finally single molecule SERS detection has allowed the study of an electrochemical phenomenon at the single molecule level and demonstrated the step-like nature of the oxidation/reduction process $[34,35]$.

In the context of the aforementioned studies, we decide to focus on one of the fundamental aspects of Raman spectroscopy: the homogeneous broadening of the Raman peaks; our goal is to see whether, once the inhomogeneous broadening brought by ensemble averaging has been eliminated, the true homogeneous broadening of the peaks and its properties and nature will be revealed. This constitutes the focus of Chapter 4.

Concerning the quest for single molecule detection of non-resonant probes by SERS, $\mathrm{C}_{60}$, with its high symmetry and remarkable spectroscopic properties regarding isotopic substitution, seems an interesting 
candidate to work with. The extension of the bi-analyte method to a multi-analyte experiment with isotopically resolved partners is the object of Chapter 5 .

Finally, because single molecule SERS studies have shown the effects that the photostability of probes have on a reliable estimation of enhancement factors and because the issue of photobleaching under SERS conditions has not yet been tackled, we dedicate the last chapter, Chapter 6 of this manuscript to the study of the photobleaching rates of resonant molecules on metallic surfaces.

These three chapters constitute the core results of this thesis. In Chapters 2 and 3 we provide details on the experimental methods and data analysis procedures which are used throughout our work. 


\section{Chapter 2}

\section{Experimental methods: instrumentation and sample preparation}

The following chapter introduces the instruments, techniques and samples which are used for the studies presented in this thesis.

\subsection{Acquiring a Raman spectrum: the Ra- man setup}

The spectrometer used in all Raman and SERS measurements is a Jobin Yvon Labram HR800 as shown on Fig. 2.1. Shown are the lasers used for our work: He-Ne laser for the $633 \mathrm{~nm}$ excitation and the Argon Ion laser for the $514 \mathrm{~nm}$ excitation. The sample is placed under the Olympus BX41 confocal microscope and the scattered light is collected in the backscattering configuration. The $(\mathrm{x}, \mathrm{y})$ stage of the microscope is motorized and can be controlled automatically by the spectrometer's software $L a b$ spec in order to carry out Raman mapping, it has a step-size of $0.1 \mu \mathrm{m}$ and a reproducibility of $1 \mu \mathrm{m}$.

A simplified diagram of the layout of the system is shown in Fig. 2.2. The laser excitation He-Ne (1) or Argon Ion (2) enters the spectrometer, plasma lines are filtered, goes through the Optical Density (OD) wheel 


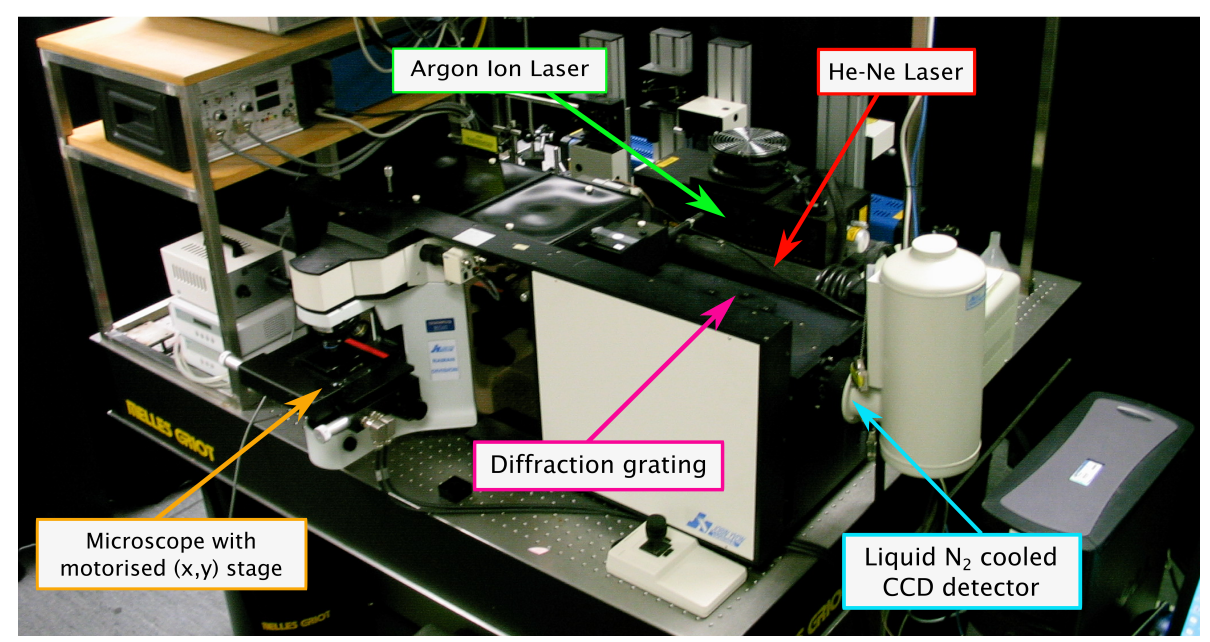

Figure 2.1: A photograph of the Horiba-Jobin Yvon Labram HR800 spectrometer in our laboratory.

which enables power modifications, and is totally reflected by the notch filter towards the sample placed under the microscope (see Fig. 2.2). The light is then focused on the sample with an objective. The scattered light is collected by the same objective and follows the same path as the incident light. While the Raman shifted scattered photons pass through the notch filter, the laser light is reflected back. The converging lens focuses the light through a pinhole slit such that an image of the sample is formed onto the pinhole. The magnification is 0.56 times the objective magnification, hence for the $\times 100$ objective, the image of a $1 \mu \mathrm{m}$ spot will have a $56 \mu \mathrm{m}$ diameter on the slit. Thus the pinhole size is limiting the region of the sample from which the signal is collected. This directly affects the spectral resolution of the experiment. The light is then sent down to a collimating mirror and onto a diffraction grating which disperses the light according to its wavelength. Finally the dispersed light is sent onto the CCD detector Symphony, which is liquid nitrogen cooled (working temperature $-133^{\circ} \mathrm{C}$ ) and offers data conversion speeds from $20 \mathrm{kHz}$ (normal mode) to $1 \mathrm{MHz}$ (ultrafast mode). 


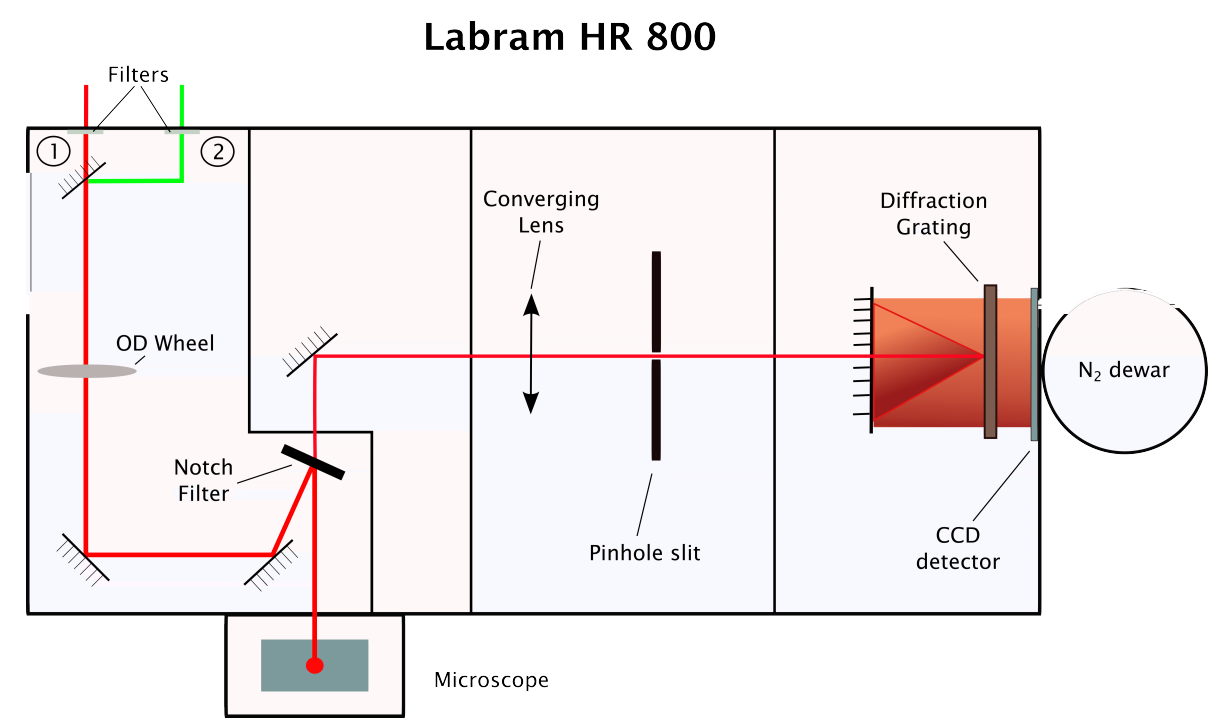

Figure 2.2: Schematic diagram of the internal optics of the Labram HR 800. The full path of the $633 \mathrm{~nm}$ (red line) laser line is shown. If we wish to use the $514 \mathrm{~nm}$ excitation, the mirror at their point of intersection needs to be lowered.

\subsection{Cryostats}

In order to get the temperature-dependent measurements of Chapter 4 and the low temperature measurements of Chapter 5 we used two types of cryostat: a closed cycle helium cryostat for the acquisition of the spectrum of the $A_{g}(2)$ mode of $\mathrm{C}_{60}$ at $9 \mathrm{~K}$ in Sec.5.4.3 and a freezing microscope stage operating with liquid nitrogen which we used for all the temperature dependent studies, down to $80 \mathrm{~K}$.

The helium cryostat is a Janis Closed Cycle Refrigerator (CCR) and enables one to reach temperatures down to $5 \mathrm{~K}$. The setup contains a copper mount (cold head) on which the sample is placed, using a highly thermally conductive silver paste. $\mathrm{C}_{60}$ in toluene was put in a glass pipette tip, sealed at both ends by Teflon tape and glued to the sample holder with silver paste. The sample is then enclosed within a windowed steel chamber and pumped down to low pressure. Once low pressure is attained, the temperature of the sample is adjusted with the cryostat. The sample is accessible to the excitation light through the windows; however micron-sized vibrations inherent to every closed cycle system are trans- 
mitted directly from the cold head to the sample, making microscopic Raman spectroscopy impossible. This is why this setup was unfortunately unsuitable for the SM-SERS studies of Chapter 4 and 5 where we needed the spatial resolution of excitation and collection through a microscope. We used a macroscopic lens to collect the scattered light in the $90^{\circ}$ configuration with the CCR cryostat (for a presentation of the $90^{\circ}$ scattering configuration: see [1]) and our other Raman spectrometer, the Jobin Yvon T 64000 is used as the cryostat is set up next to it.

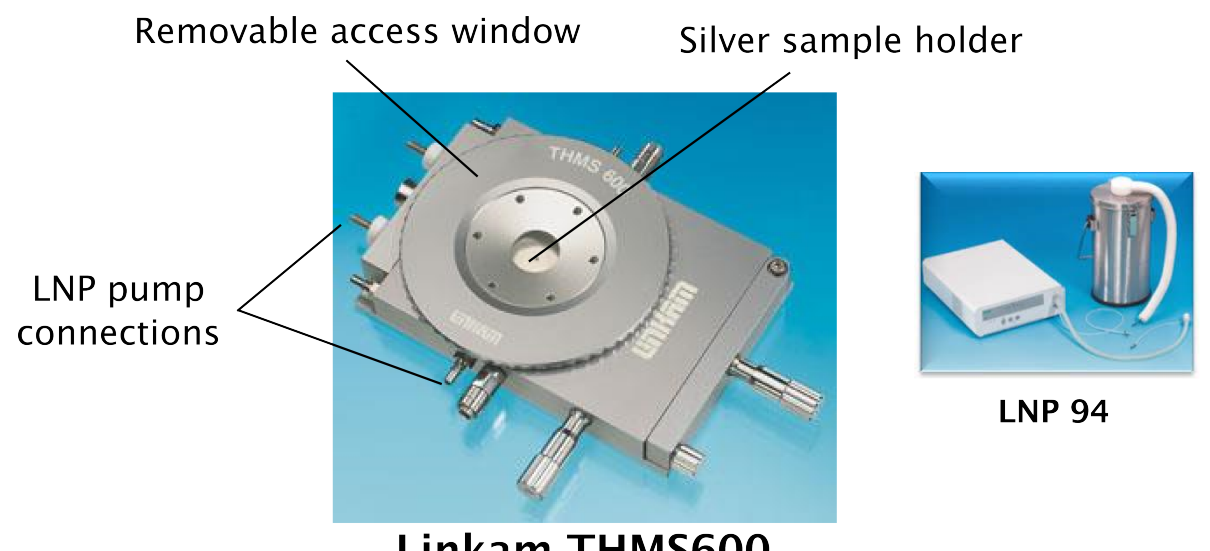

Figure 2.3: Linkam THMS600 microscope stage

The microscope stage we used for the temperature dependence of the homogeneous broadening of Nile blue in Chapter 4 and the low temperature SERS measurements of $\mathrm{C}_{60}$ in Chapter 5 is a Linkam THMS600 which is cooled by liquid nitrogen (the liquid $\mathrm{N}_{2}$ pump is the LNP 94 model). It is a compact bench-top apparatus which enables microscopic acquisitions of Raman spectra at temperatures ranging from $-195{ }^{\circ} \mathrm{C}$ to $600{ }^{\circ} \mathrm{C}$ of a sample enclosed into the stage (see Fig. 2.3). We used it in the lower temperature range, from $80 \mathrm{~K}$ to room temperature. The sample is sitting on a highly polished silver element through which liquid or cold nitrogen is circulating. The LNP pumps the liquid nitrogen from a $2.5 \mathrm{~L}$ Dewar into the circuit and diverts a part of it as nitrogen gas into the chamber. This can be used to purge the atmosphere of the chamber prior going down to low temperature by evacuating air (and thus water) from the chamber and then preventing condensation of water or crystallization 
of ice onto the sample. The controller enables one to chose a target temperature and to reach it with speeds as fast as $130{ }^{\circ} \mathrm{C} \mathrm{min}^{-1}$ or as low as $0.1^{\circ} \mathrm{C} \mathrm{min}{ }^{-1}$ by adjusting the rate of the liquid nitrogen pump. Because the sample is enclosed into the chamber, we need to use objectives with a long working distance and hence a lower numerical aperture, like the $\times 100$ Long Working Distance (LWD) with N.A.=0.6.

\subsection{SERS substrates}

One of the main factors of success (or conversely, of failure!) in SERS experiments is the SERS substrate itself, whether it offers high, medium or low enhancement locations, whether these locations are sparse or many (the SERS enhancement factor distribution is important [36]), its homogeneity and last but not least, its affinity with the SERS analyte we want to use. Then, from the point of view of the "user", it should be easy to prepare and, ideally, be reproducible.

Our group has been mainly exploiting three types of SERS substrates: planar metallic surfaces in the Kretschmann configuration [37, 38] (where the enhancement of the Raman signal comes from the coupling of the Raman scattering to the surface plasmon polaritons (SPP) excited at the metal-air interface), aggregated colloidal solutions, and aggregated colloids on planar substrates. The latter are systems presenting many hot spots which are typically located at the gap junctions between metallic nanostructures. Aggregated metallic colloidal solutions are among the most sensitive SERS substrates available [39, 40, 41] where the SERS enhancement factor can be as high as $\sim 10^{9}-10^{11}[42,13]$ for silver nanoparticles in solution.

The studies presented in Chapters 4 and 5 make use of SM-SERS capabilities to study the homogeneous broadening of a vibration of a well known dye and to resolve individual cases of isotopic substitution induced frequency shifts in $\mathrm{C}_{60}$ in the latter. Our group conducted most of its extensive work on SM-SERS with silver colloids prepared in situ- in particular Lee \& Meisel [43] colloids - as SERS substrate and a lot of work was done previously on resonant dyes (rhodamine 6G, Nile blue, Crystal 
violet) and Lee \& Meisel colloids [23, 25, 29]. Consequently, our group is familiar with the sample preparation for SM-SERS detection with such systems and the statistics of the corresponding measured SERS signals.

\subsubsection{Silver colloids as SERS substrates}

Silver colloids are extensively used in the SERS context even though they are more susceptible to oxidation under ambient conditions than gold colloids and the chemistry of molecular binding to them is not as well understood [1]. Nevertheless, silver particles give rise to much larger enhancements in the visible with plasmon resonances for Ag aggregates located typically at around 600 to $650 \mathrm{~nm}$ as can be measured by UVvisible extinction spectroscopy. The following section presents the recipes for preparation of Ag colloids that were used for SERS and SM-SERS work for this thesis.

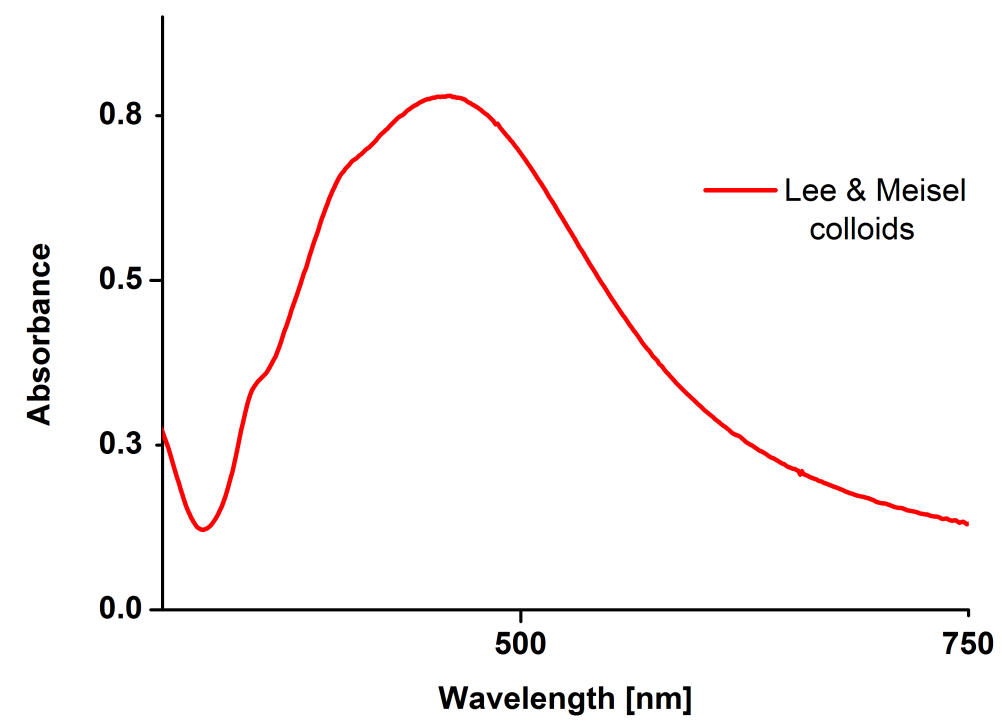

Figure 2.4: UV-Vis absorption spectrum of the Lee \& Meisel Ag colloids (non-aggregated) diluted twice, typically used for Single Molecule SERS experiments. 


\section{Citrate-reduced silver colloids: the Lee \& Meisel method}

The preparation of Lee \& Meisel silver colloids [43] is rather simple and its characteristics as SERS substrates have been studied in detail [44]. I will just summarize here the essential steps of the process:

- $90 \mathrm{mg}$ silver nitrate $\left(\mathrm{AgNO}_{3}\right)$ is added to $500 \mathrm{~mL}$ of ultrapure water in a $1 \mathrm{~L}$ conical flask equipped with a magnetic stirring bar. The solution is then brought to boil.

- Upon boiling, $10 \mathrm{~mL}$ of trisodium citrate $1 \% \mathrm{Na}_{3} \mathrm{C}_{6} \mathrm{H}_{5} \mathrm{O}_{7}$ (100 mg, $294.1 \mathrm{~g} \mathrm{~mol}^{-1}$ ) is added drop-wise and the solution is kept boiling under vigorous stirring for approximately 1 hour.

- The solution is then allowed to cool down to room temperature and eventually stored in a plastic container wrapped in aluminum foil to protect it from sunlight, lest the particles start to oxidize [45].

After addition of the reducing agent (trisodium citrate), the solution rapidly undergoes colour changes: from transparent to clear brown to eventually a grey-green opaque coloration. The silver particles are coated by citrate ions $\mathrm{C}_{6} \mathrm{H}_{5} \mathrm{O}_{7}^{3-}$ which in this case play both roles of reducing and stabilizing agent; they indeed prevent the particles from aggregating by turning on Coulomb repulsion.

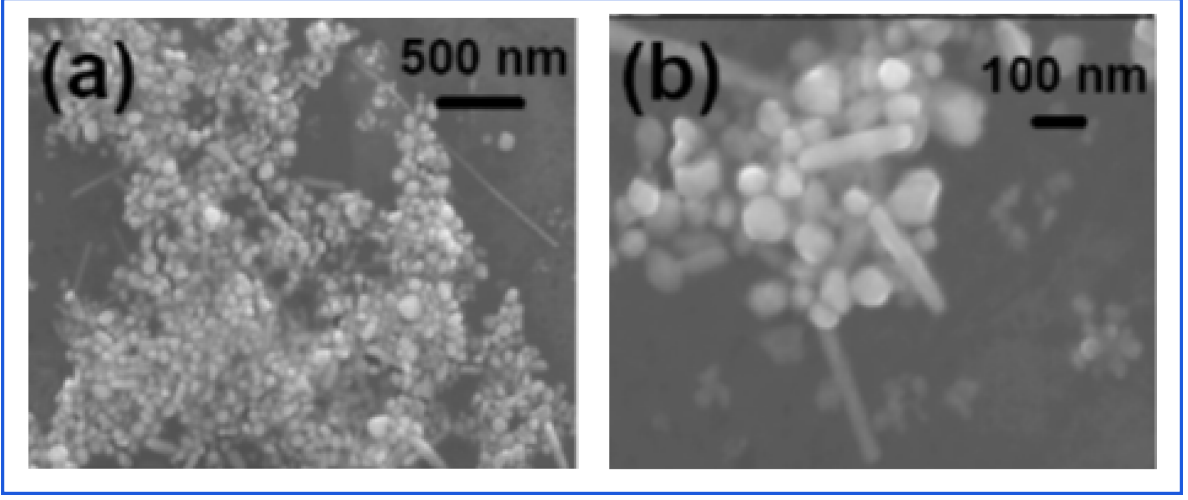

Figure 2.5: Scanning electron microscope (SEM) images of citratereduced Lee \& Meisel silver colloids. One can appreciate the polydispersity in sizes and shapes, particularly the presence of rods. The spherical particles display an average diameter of $60 \mathrm{~nm}$. 
The UV/Vis absorption spectrum of Lee \& Meisel colloids shows an absorption maximum at $\sim 430-460 \mathrm{~nm}$ as shown in Fig. 2.4. The main peak is the contribution of the dipolar localized surface plasmon resonances of the individual particles; this peak is strongly broadened in the case of such colloids due to the high polydispersity in sizes and shapes (see Fig. 2.5), typically spherical particles and rods are produced. The shoulder at $\approx 380 \mathrm{~nm}$ can be attributed to the quadrupolar resonance. The mean particle size is found to be around $60 \mathrm{~nm}$ in diameter, thus if we assume the reduction of the $\mathrm{AgNO}_{3}$ has been complete and that all the silver is in the form of spheres of $60 \mathrm{~nm}$ diameter then the colloid concentration can be estimated to be $\approx 10^{10}$ particles $/ \mathrm{cm}^{3}$ as one $60 \mathrm{~nm}$ diameter silver sphere contains about $3.66 \times 10^{-17}$ moles of $\mathrm{Ag}$ and the starting $\mathrm{Ag}$ concentration is $\approx 1 \mathrm{mM}$ which leads to an estimate of $\approx 2.73 \times 10^{10}$ particles $/ \mathrm{cm}^{3}$. This is most likely an overestimate of the real concentration as a large proportion of silver could be in a small number of very large particles (SEM pictures of citrate-reduced colloids show that there is a significant proportion of nanorods also produced in the process).

Lee \& Meisel silver colloids are negatively charged since they are coated with (and bathing in) the unreacted citrate ions $\mathrm{C}_{6} \mathrm{H}_{5} \mathrm{O}_{7}^{3-}$ which produce a repulsive Coulomb interaction between the particles and ensure the colloidal stability. The single colloids dispersed in water already provide some SERS enhancement through the excitation of their dipolar localized surface plasmon resonances at $\sim 430-460 \mathrm{~nm}$ as seen in Figure 2.4 , providing a maximum enhancement factor which can be calculated via Mie theory to be $\sim 1.6 \times 10^{5}$ for a single $50 \mathrm{~nm}$ silver sphere in water. Even though this enhancement is not negligible, it may yet not be large enough to enable the detection of single molecules under SERS conditions; moreover the resonance wavelength is too close to the UV range for a convenient application.

It is common practice when carrying out SERS experiments, while using metallic colloids as a source of electromagnetic enhancement, to break the metastable equilibrium of the system by adding some charged species into the solution and thus screening the Coulomb repulsion be- 
tween the particles. This results in partial aggregation of the colloids solution which is desirable as the largest SERS enhancements generally occur at the junction between two particles. In the case of the citratereduced silver colloids used throughout this study, this is achieved by mixing the Ag colloids with potassium chloride $(\mathrm{KCl}) 20 \mathrm{mM}$ in 1:1 proportion, allowing the silver particles to get close enough to each other for them to feel the van der Waals attraction forces, the colloidal solution remains stable and shows good SERS activity [46]. The chloride ions also replace the citrate ions on the surface of the silver nanoparticles [47]. The solution is typically allowed to aggregate for 15 minutes before being used for the SERS experiments.

To summarize this section on the Lee \& Meisel colloids, we can say that, for us their advantages are:

* They are easy to make. In fact it takes only about an hour and a half to synthesize them, no advanced chemical facility is required as there is no need for a fume-hood and the reactants are not dangerous nor toxic.

* We know exactly in our group how to prepare them for SERS or SM-SERS and understand them well, especially when they are used in combination with resonant dyes like rhodamine $6 \mathrm{G}$ or Nile blue.

They nevertheless have several significant drawbacks:

* Their properties can vary quite a lot from one batch to the other. These colloids are also not very stable and degrade over time. A good batch may last $\approx 1$ year in the refrigerator.

* They contain some very large particles which can play some important role in our experiments. Generally speaking, their size and shape polydispersity makes them a very messy system and is an obstacle to a proper understanding of the surface coverage of the colloids by the analyte. 


\section{Borohydride reduced silver colloids}

As an alternative to Lee \& Meisel colloids, we prepared borohydride reduced silver colloids following the method given in [48] but adding $\mathrm{KCl}$ as a first step to increase stability.

- $\mathrm{KCl}\left(7.9 \mathrm{mg}, 74.6 \mathrm{~g} \mathrm{~mol}^{-1}\right)$ is added to a solution of $\mathrm{AgNO}_{3}(18 \mathrm{mg}$, $1.06 \mathrm{mM}$ ) in $80 \mathrm{~mL}$ ultrapure water in a $250 \mathrm{~mL}$ round-bottomed flask equipped with a stir bar. This results in a cloudy blue precipitate of $\mathrm{AgCl}$.

- The solution is cooled in an ice bath and an ice-cooled solution of $\mathrm{NaBH}_{4}\left(6 \mathrm{mg}, 37.8 \mathrm{~g} \mathrm{~mol}^{-1}\right)$ in ultrapure water is added drop-wise to the $\mathrm{AgCl}$ solution. Upon addition of the reductant, the solution becomes a cloudy yellow/grey color.

- The solution is left to warm to room temperature while being kept stirring under a fume hood for at least 1 hour to react the excess $\mathrm{NaBH}_{4}$.

The colloids synthesized via this method are typically smaller than those from the Lee \& Meisel method; their advantage over the latter is that the silver particles are not coated by citrate, thus eliminating the problem of carbon contamination of the SERS signal, which can be a significant problem when one transfers colloids onto planar SERS substrates by drying them. However, borohydride reduced silver colloids are much less stable than the Lee \& Meisel colloids and their shelf lifespan does not exceed a couple of weeks at best.

Borohydride reduced silver colloids were used for the preparation of the planar SERS substrate for the experiments of Chapter 4.

\section{Hydroxylamine reduced silver colloids}

We also synthesized hydroxylamine hydrochloride reduced silver colloids according to the method (C) proposed by Leopold \& Lendl [49].

- A solution of $\mathrm{AgNO}_{3} 10^{-2} \mathrm{M}$ is prepared by dissolving $42.4 \mathrm{mg}$ of silver nitrate in $25 \mathrm{~mL}$ of ultrapure water. 
- A solution $0.96 \mathrm{M}$ of $\mathrm{NaOH}$ is prepared by dissolving $96.6 \mathrm{mg}$ of sodium hydroxide in $25 \mathrm{~mL}$ of ultrapure water.

- A solution of hydroxylamine hydrochloride $1.67 \mathrm{mM}$ in $3.33 \mathrm{mM}$ $\mathrm{NaOH}$ is prepared by dissolving $11.6 \mathrm{mg}$ hydroxylamine with $3.45 \mathrm{~mL}$ of the previous $\mathrm{NaOH}$ solution and water into a volume of $100 \mathrm{~mL}$. The aim is to get a concentration ratio between hydroxylamine and $\mathrm{NaOH}$ of $1: 3$.

- $1 \mathrm{~mL}$ of the $\mathrm{AgNO}_{3}$ solution is added rapidly with a pipette to $9 \mathrm{~mL}$ of hydroxylamine in $\mathrm{NaOH}$. The reaction is instantaneous and the solution turns to a bright yellow/orange colour.

The advantages are that they are highly reproducible, the monodispersity is improved compared to Lee\& Meisel silver colloids and their shelf-life is longer (couple of months). the disadvantage is that we have less experience in using them for SERS, thus some work is required to optimize the parameters.

\subsubsection{Preparation of planar SERS substrates: the dried colloids approach}

This kind of substrate is very easy to fabricate: it can be as easy as drying a drop of colloidal solution on a glass slide or silicon wafer and the aggregation will eventually occur upon drying. Thanks to this aggregated state, such substrates usually exhibit large SMEF and average EF due to the presence of many gap-coupled plasmon resonances at the junction between particles [50]. On the other hand, due to the randomness of the fractal-like structure of dried colloids, the SERS enhancement is very non-uniform (especially when using high magnification objectives)and susceptible to dramatic changes with very small changes in the geometry of the structures. Hence it is important to have a statistical criterion for the single molecularity of the detected signals firstly, and secondly, a way to prevent the colloids from moving once they are dried onto the substrate. For the latter goal, we coated our substrates (whether glass, 


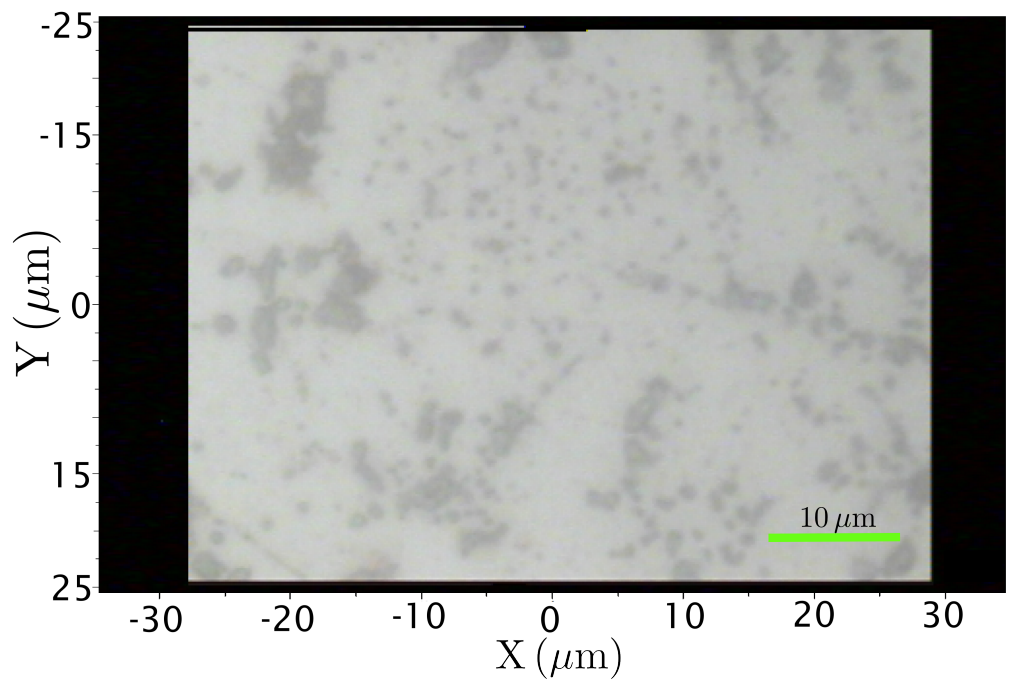

(a) Dried Lee \& Meisel silver colloids

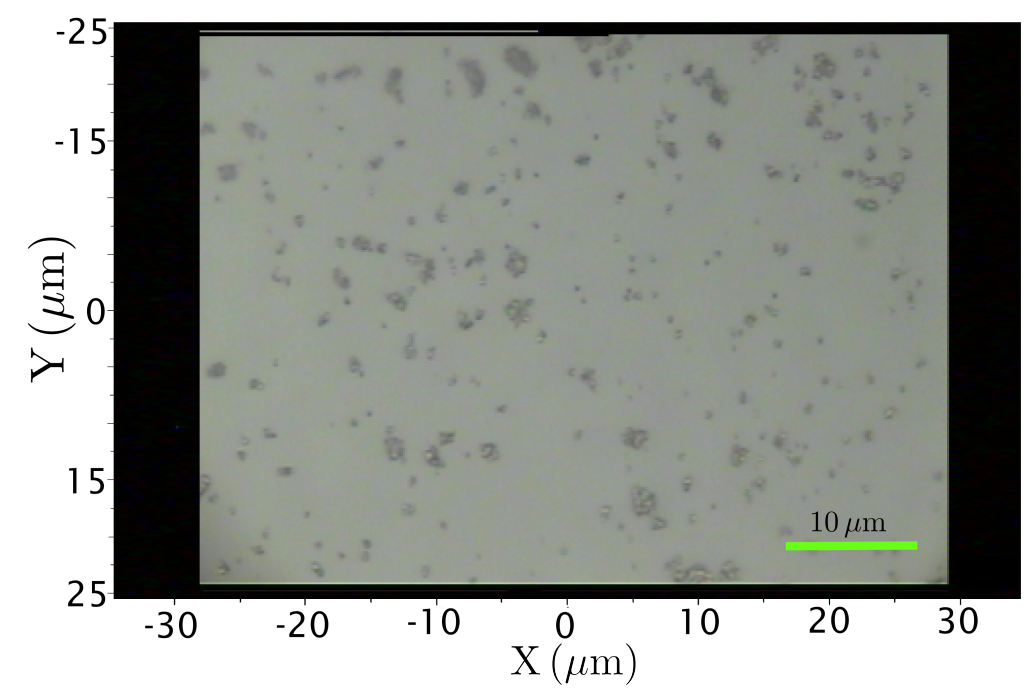

(b) Dried borohydride reduced silver colloids

Figure 2.6: Photographs of the surface of the SERS substrates obtained by drying (a) Lee \& Meisel colloids $+20 \mathrm{mM} \mathrm{KCl}$ on poly-L-lysine coated $\mathrm{Si}$ and (b) borohydride reduced silver colloids $+10 \mathrm{mM} \mathrm{KCl}$ on poly-Llysine coated Si. The pictures were taken with the optical microscope $(\times 100)$ and white light. 
quartz or silicon) with a poly-L-lysine layer to graft the colloids to the surface.

\section{Deposition of poly-L-lysine layer on the substrate}

Poly-L-lysine is a small natural polymer of the amino acid L-lysine and is used as a charge enhancer for promoting the attachment of negatively charged species to a surface. Poly-L-lysine (30 to $70 \mathrm{kDa}$ ) was purchased from Sigma-Aldrich. The process we used to cover substrates (glass or silicon wafer) is as follows:

- A buffer solution $0.1 \mathrm{mM}$ Tris- $\mathrm{HCl}$ is prepared. Tris- $\mathrm{HCl} 0.1 \mathrm{M}$ was purchased from Sigma Aldrich.

- A stock of "pre-poly" solution is prepared by adding $0.643 \mathrm{~g}$ of $\mathrm{NaCl}$ to $110 \mathrm{~mL}$ of the buffer solution prepared above. The $\mathrm{pH}$ is tested with $\mathrm{pH}$ strips to be $\approx 7$. The addition of $\mathrm{NaCl}$ is necessary in order to preserve the structure of the polypeptide.

- The substrates (glass or $\mathrm{Si}$ ) are cleaned in an $\mathrm{O}_{2}$ plasma cleaner.

- A solution of poly-L-lysine is pepared by dissolving polylysine $1 \mathrm{mg} / \mathrm{mL}$ in the pre-poly solution. The poly-L-lysine powder is put first in a glass beaker then the pre-poly solution is poured onto it.

- The substrate is then immersed in the beaker and let to sit for 30 min.

- The substrate is then rinsed in the buffer solution to remove excess unattached poly-L-lysine and dried under $\mathrm{N}_{2}$ flux.

The SERS substrates are then prepared by placing a drop of silver colloids containing the analyte for 5 minutes then siphoning off the drop. Fig. 2.6 shows photographs of substrates prepared with dried Lee \& Meisel colloids (a) and borohydride reduced silver colloids (b) on silicon wafers coated with poly-L-lysine. The dried Lee \& Meisel $+\mathrm{KCl}$ tend to result in bigger, less uniform aggregates drying in fractal-like structures and citrate are expected to be everywhere. The dried borohydride 
reduced silver colloids $+10 \mathrm{mM} \mathrm{KCl}$ result in much smaller and sparser aggregates.

\subsection{SERS active dyes under study}

The work presented in Chapter 4 has been carried out on two dyes, measured in resonance conditions : Nile blue A (NB) and rhodamine 800 (R800) (see Figure 2.7). Both dyes were purchased from Sigma-Aldrich.<smiles>CC[N+](CC)=c1ccc2nc3c(cc(N)c4ccccc43)oc-2c1</smiles>

(a) Nile Blue A

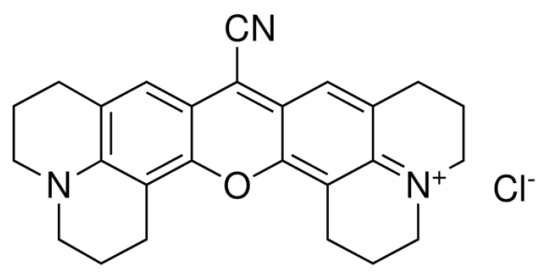

(b) Rhodamine 800

Figure 2.7: Chemical structure of (a) Nile blue A, here with a counter perchlorate ion and (b) rhodamine 800 with a counter ion $\mathrm{Cl}^{-}$.

Nile blue $\left(\mathrm{C}_{20} \mathrm{H}_{20} \mathrm{~N}_{3} \mathrm{O}^{+}\right.$, molar mass $\left.353.85 \mathrm{~g} \mathrm{~mol}^{-1}\right)$ has been extensively studied as a SERS probe by our group [51, 52, 53] over the past years : it is soluble in both water and ethanol and has its maximum optical absorption at $635 \mathrm{~nm}$ in water which closely matches the $633 \mathrm{~nm}$ emission wavelength of the He-Ne laser used throughout the experiments and thus ensures one benefits from the further enhancement of the Raman signal due to resonant excitation of the molecules. In order to avoid dilution errors, care had to be taken to dissolve the powder in a large volume of water, typically by preparing (under long-lasting and vigorous stirring) $5 \mathrm{~L}$ of $15 \mu \mathrm{M}$ Nile blue solution.

Rhodamine 800 is a laser dye belonging to the same class as the widely used (and studied) rhodamine 6G and is a common stain in the study of mitochondria. It has the chemical formula $\mathrm{C}_{26} \mathrm{H}_{26} \mathrm{~N}_{3} \mathrm{O}^{+}$and a molar mass of $396.21 \mathrm{~g} \mathrm{~mol}^{-1}$. What makes R800 an interesting system to study in the case of Raman spectroscopy is the presence of a cyano bond $\mathrm{C} \equiv \mathrm{N}$ which forms a localized high frequency Raman active vibration at $2230 \mathrm{~cm}^{-1}$. 
In this region, only triple bonds have Raman active vibrations. Being localized, the cyano stretching eigenvector is particularly susceptible to isotopic substitution and such a substitution (for example ${ }^{13} \mathrm{C} \longrightarrow{ }^{12} \mathrm{C}$ ) can readily be seen as a detectable shift in the frequency of the mode on a single molecule spectrum [54].

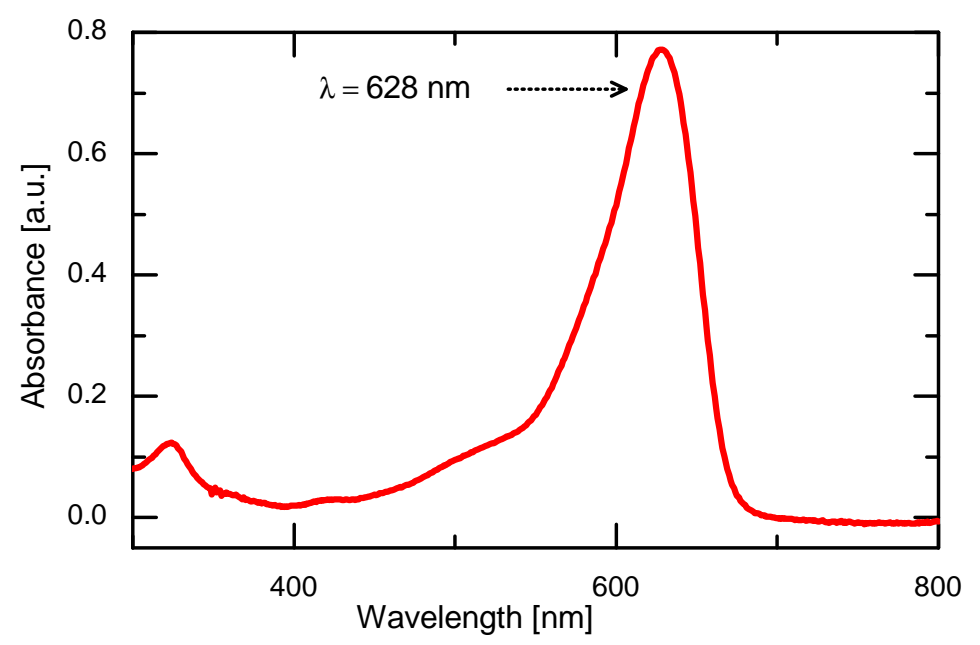

(a) Nile blue A absorption spectrum

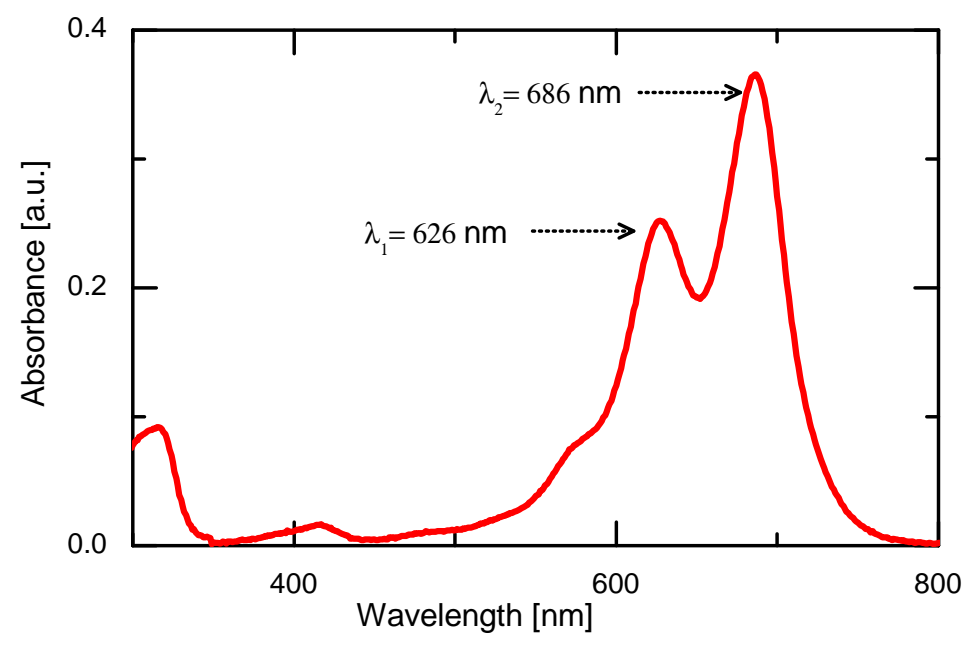

(b) Rhodamine 800 absorption spectrum

Figure 2.8: UV-Visible absorption spectra of Nile blue A (a) and rhodamine 800 (b) in water. Note the presence of two resonance absorption peaks for rhodamine 800; with $\lambda_{1}$ corresponding to the absorption due to the dimers of rhodamine 800 and $\lambda_{2}$ to the monomers [55]. 


\section{Chapter 3}

\section{Data analysis}

As we will see in Chapter 4, the spectrum around a Raman mode of an isolated molecule is expected to have a Lorentzian lineshape. However, when recording experimental spectra, many additional sources of broadening of the Raman signal arise, such as ensemble averaging of the signal coming from many molecules which experience different environmental conditions or speeds and instrumental broadening, in particular the fact that spectral resolution is limited. In most of the cases, when we carry out Raman spectroscopy or SERS experiments, we do not try to extract from the data the physical reasons for the broadening of the signal; we are interested in determining various relevant parameters which will help us describe and understand the physics of our system. We perform fits of the data to a model or a reference in order to obtain:

- The intensity of a Raman peak without the dependence on the noise.

- Central frequencies and linewidths automatically and precisely.

- In some cases the lineshape of a peak.

Two approaches were used throughout this work to the fitting of Raman and SERS spectra: non-linear least square fits to a model function (pseudo-Voigt in most of the cases) and fits of the data to a linear combination of reference spectra. 


\subsection{Non linear least-square fits of the Ra- man peaks to pseudo-Voigt profiles.}

It is common in spectroscopy to fit the intensity profile of peaks to Voigt or pseudo-Voigt profiles [56]. This combines either by convolution (Voigt) or by simple addition (pseudo-Voigt) a Lorentzian and a Gaussian function. The Lorentzian mainly describes the homogeneous or natural broadening (when lifetime effects dominate) while the Gaussian contributes more to rendering the inhomogeneous broadening mechanisms, sample imperfections and any random statistical effect, whatever their origin.

The Voigt lineshape is defined by:

$$
V\left(\omega-\omega_{0}, \sigma, \Gamma\right)=\int_{-\infty}^{+\infty} \frac{e^{-t^{2} /\left(2 \sigma^{2}\right)}}{\sigma \sqrt{2 \pi}} \frac{\Gamma / 2}{\left(\omega-\omega_{0}-t\right)^{2}+(\Gamma / 2)^{2}} d t
$$

The function $V$ above is normalized and centered in $\omega_{0}$.

In the case of the Pseudo-Voigt function, the convolution is approximated by the weighted sum:

$$
V_{\text {pseudo }}(\omega)=\alpha G(\omega)+(1-\alpha) L(\omega)
$$

$G$ and $L$ being respectively a Gaussian and a Lorentzian function and with $\alpha \in[0,1]$ the Gaussian fraction.

The purpose here with these pseudo-Voigt fits is not to give a physical interpretation to $\alpha$. The main advantage of Voigt (or pseudo-Voigt) fits is that the combination of Lorentzian and Gaussian can be tailored to suit the particular peak shape of a particular spectrum. In any case, in Raman spectroscopy, one rarely has the sufficient intensity and resolution to measure the real shape of a spectroscopic peak.

In this thesis, every time the data around a Raman peak centered in $\overline{\nu_{0}}$ were fitted to a Voigt profile (by non-linear least-squares fit), they were 
fitted to the equation:

$$
\begin{aligned}
\frac{I(\bar{\nu})}{I_{\max }}= & \alpha \exp \left[-4 \ln (2)\left(\frac{\bar{\nu}-\overline{\nu_{0}}}{\Gamma}\right)^{2}\right] \\
& +(1-\alpha) \frac{(\Gamma / 2)^{2}}{\left(\bar{\nu}-\overline{\nu_{0}}\right)^{2}+(\Gamma / 2)^{2}}+B(\bar{\nu}) .
\end{aligned}
$$

where $\Gamma$ is the full width at half maximum (FWHM) of the peak, B is a linear background in the neighborhood of $\overline{\nu_{0}}: B(\bar{\nu})=a \bar{\nu}+b$. This definition was chosen such that the FWHM of the Gaussian and the Lorentzian are the same $(\Gamma)$.

$\Gamma$ is related to the Gaussian standard deviation $\sigma$ by:

$$
\Gamma=2 \sqrt{2 \sigma \ln 2}
$$

The way we proceed is by doing a non-linear least squares fit of the data around the peak to the profile given in Eq. 3.4. If the experimental data set of $n$ points (recorded spectrum) is $\left(\bar{\nu}_{i}, S_{i}\right)_{i=1 \ldots n}$, and the model function $f(\bar{\nu}, \vec{\alpha})$ (given in Eq. 3.4 above, $\vec{\alpha}$ is a vector holding the adjustable parameters of the fit), the principle is to minimize the sum $\Sigma$ of squared residuals $\Sigma=\sum_{i=1}^{n}\left(S_{i}-f\left(\bar{\nu}_{i}, \vec{\alpha}\right)\right)^{2}$ by adjusting the parameters of $\vec{\alpha}$. The minimum of $\Sigma$ is found by setting the gradient of $\Sigma$ to 0 , which leads to solving $m$ gradient equations if there are $m$ parameters:

$$
-2 \sum_{i=1}^{n}\left(S_{i}-f\left(\bar{\nu}_{i}, \vec{\alpha}\right)\right) \frac{\partial f\left(\overline{\nu_{i}}, \vec{\alpha}\right)}{\partial \alpha_{j}}=0 \quad \text { for } j=1 \ldots m
$$

As $f$ is not linear, initial values or guess have to be chosen for the parameters at the start. The parameters $\alpha_{j}$ are then refined iteratively using an optimization algorithm. In the case of Microsoft Excel (used for most of the non-linear fits of this thesis), we use the Solver to minimize the sum of squared residuals. The Solver uses a method called the Generalized Reduced Gradient (GRG) non-linear engine which is suitable for smooth non-linear problems [57]; this method is also used by the optimization algorithm of fminsearch in Matlab. It can find a locally optimal solution to a well-scaled problem. The boundaries of the fit, the interval $\left[\overline{\nu_{1}}, \overline{\nu_{2}}\right]$, 
are important as $\Sigma$ has to be convex over $\left[\overline{\nu_{1}}, \overline{\nu_{2}}\right]$. Indeed, if the problem is not well-scaled, the algorithm might stop after having reached a local optimum which could be different from the global optimum solution.
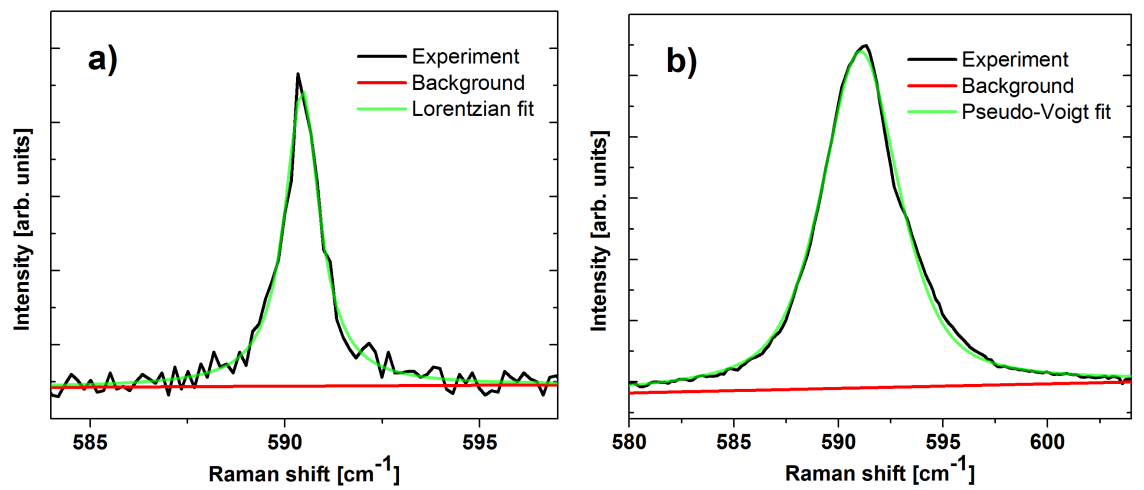

Figure 3.1: Examples of non-linear least square fits of spectra in the $590 \mathrm{~cm}^{-1}$ region of a sample of Nile blue in SERS conditions at low temperature $(80 \mathrm{~K})$. The spectrum in a) is a single molecule event and the best fit was obtain by fitting the data to a Lorentzian lineshape. The spectrum in b) is the average SERS spectrum and one can appreciate the broadening induced by ensemble-averaging of the signal. The best fit was obtained by fitting to a pseudo-Voigt profile with $\alpha=0.4$.

In the case of the fitting of experimental Raman (or SERS) spectra to pseudo-Voigt profiles, the adjustable parameters are the central frequency $\overline{\nu_{0}}$, the peak height $I_{\max }$, the linear background $(a, b)$, the parameter $\alpha$ (proportion of Gaussian in the pseudo-Voigt model) and the FWHM. We fix the parameter $\alpha$ most of the time or manually look for the best $\alpha$ value as, in the case of noisy signal, it can easily converge towards nonphysical solutions. For the fits of SM-events in Chapter 4, $\alpha$ was fixed to be 0 as a pure Lorentzian lineshape for these spectra was expected. Generally we do not want to assign a physical meaning to the width $\Gamma$ either except in the Single Molecule cases where $\Gamma(T)$ gives us the temperature dependence of the broadening of the $590 \mathrm{~cm}^{-1}$ mode of Nile blue. In our work, we have used these non-linear least squared fits mainly as a way to identify SM-events in series of thousands of spectra when linear fitting (see Section 3.2) is not possible because no reference can be 
used or the peak's width and position can vary a lot from one spectrum to another as it is the case in the experiments of Chapter 4. For example, $\Gamma$ can range from 0.9 to $3 \mathrm{~cm}^{-1}$ at $80 \mathrm{~K}$ for the Nile Blue mode at $590 \mathrm{~cm}^{-1}$ and we can see also that the SM-events span 5 to $6 \mathrm{~cm}^{-1}$ in position. Non-linear least square fits were also used to determine the width or position of a given peak in a spectrum or to fit data when determining scattering volumes (see Section 5.5.3).

\subsection{Linear fit of a spectrum to a reference}

The main inconveniences of the non-linear least square fits presented above are that they are slower than linear fits. The quality of the fit becomes increasingly bad when the signal-to-noise ratio of the spectrum is low, they can not take into account any asymmetry of the peak and are quite unstable when trying to fit multiple peaks in the same spectral region. This is why, sometimes, we prefer to do a linear fit of the data; for example when we expect the spectrum to be a linear combination of one or more reference spectra and a background. Linear fits are very fast and have a unique solution (mathematically), which in most cases is also the physically acceptable solution.

Let us consider the case of an experimental spectrum $S(\lambda)$ that we want to fit to a reference spectrum $R(\lambda)$ and a linear background $B(\lambda)$. We want the spectrum $S(\lambda)$ written as:

$$
\begin{aligned}
S(\lambda) & =\alpha_{1} R(\lambda)+B(\lambda) \\
& =\alpha_{1} R(\lambda)+\alpha_{3} \lambda+\alpha_{2}
\end{aligned}
$$

If we write $S(\lambda)$ as a vector $\left(S(\lambda)_{j}\right)_{j \in[1 \ldots N]}$ with $N$ the total number of pixels recorded (which is 1024 with our CCD detector) then Eq. 3.7 becomes:

$$
\left(\begin{array}{c}
S\left(\lambda_{1}\right) \\
\vdots \\
S\left(\lambda_{N}\right)
\end{array}\right)=\left(\begin{array}{ccc}
R\left(\lambda_{1}\right) & 1 & \lambda_{1} \\
\vdots & \vdots & \vdots \\
R\left(\lambda_{N}\right) & 1 & \lambda_{N}
\end{array}\right)\left(\begin{array}{c}
\alpha_{1} \\
\alpha_{2} \\
\alpha_{3}
\end{array}\right)
$$


If we write:

$$
A=\left(\begin{array}{ccc}
R\left(\lambda_{1}\right) & 1 & \lambda_{1} \\
\vdots & \vdots & \vdots \\
R\left(\lambda_{N}\right) & 1 & \lambda_{N}
\end{array}\right)
$$

then doing a linear fit of the spectrum $\mathrm{S}$ is solving the overdetermined system of $N$ equations and 3 unknowns $\vec{\alpha}=\left(\alpha_{1}, \alpha_{2}, \alpha_{3}\right)$. To solve this system, we use the command mldivide in Matlab; mldivide $(A, S)$ will return a least-squares solution of this system by minimizing the function $\Sigma:$

$$
\Sigma(\vec{\alpha})=\sum|S-A \vec{\alpha}|^{2}
$$

This problem has an unique solution if the columns of $A$ are linearly independent i.e. the rank of $A$ is 3 . The minimization problem is then equivalent to solving the system of 3 equations, $k=1 \ldots 3$ :

$$
\begin{aligned}
0=\frac{\partial \Sigma}{\partial \alpha_{k}} & =\sum_{j=1}^{N}-2 A_{j k}\left(S_{j}-\sum_{i=1}^{3} A_{j i} \alpha_{i}\right) \\
& =\sum_{j=1}^{N}\left(-2 A_{j k} S_{j}+2 \sum_{i=1}^{3} A_{j k} A_{j i} \alpha_{i}\right) \\
& =\sum_{j=1}^{N}\left(-2 A_{j k} S_{j}\right)+2 \sum_{j=1}^{N} \sum_{i=1}^{3}\left(A_{k j}^{t} A_{j i} \alpha_{i}\right) \\
& =\sum_{j=1}^{N}\left(-2 A_{k j}^{t} S_{j}\right)+2 \sum_{i=1}^{3}\left(A^{t} A\right)_{k i} \alpha_{i} \\
& =\left(2 A^{t} S\right)_{k}+\left(A^{t} A \vec{\alpha}\right)_{k}
\end{aligned}
$$

with $A^{t}$ the transpose of $A$. $A^{t} A$ is then a square $3 \times 3$ matrix. As $A$ is of $\operatorname{rank} 3, A^{t}$ is also of rank 3 and thus $A^{t} A$ is of rank 3 so invertible. Hence finding the best fitting parameters in the least-squares sense is solving the equation:

$$
\vec{\alpha}=\left(A^{t} A\right)^{-1} A^{t} S
$$


There is only one $\vec{\alpha}$ satisfying Eq. 3.11. Note: the background $B(\lambda)$ can be chosen to be quadratic or more generally a polynomial of order $n$ and $M$ reference spectra can be also chosen; then $A$ is a $n+M+1$ by $N$ matrix and is required to have a rank of $n+M+1$ for the least-squares problem to have a unique solution.

This is very fast because the derivative of $f$ is linearly dependent on the $\alpha_{k}$ and hence can be used to fit large series of spectra. It is for example particularly suitable for the analysis of bi-analyte experiments $[25,23]$ where each SERS spectrum taken from a mixture of two or more analytes is compared to the reference spectra of the analytes.

It is important to note here that this particular kind of linear fit does not work if the broadening of the sample spectra (FWHM) and/or the central frequency of the peaks are different from the reference spectrum. Hence, approximating the sample spectra to a linear combination of reference spectra is not valid in case of significant frequency shifts and/or change in broadening; this is the case when the linewidth of the mode is particularly small compared to the frequency shifts induced by external perturbations as we see later in Chapter 4 or when the expected frequency position of the peak can span a wide range of wavenumbers as is the case with the isotopically enriched sample of $\mathrm{C}_{60}$ in Chapter 5 . The aim of the data analysis of the $\mathrm{C}_{60}$ experiments was mainly to discriminate single molecule events among tens of thousands of spectra of $\mathrm{C}_{60}$ on silver, most of them showing no signal or massive and complicated backgrounds (due probably to the presence of amorphous carbon caused by burning the surface when the local field intensity was too high); and the spectra showing the presence of the $1470 \mathrm{~cm}^{-1}$ peak had usually also complicated non-linear backgrounds. Non-linear least-squares fits to Voigt profiles were unsuccessful in filtering SM-SERS or simply SERS events as the algorithm would often try to fit noise oscillations or parts of the background as peaks. Because of the complicated features of the spectra of this experiment, we adapted the linear fitting routine to the situation as shown below. 


\subsection{Improvement of the linear fits}

In Chapter 5, we focus on the pentagonal pinch mode $A_{g}(2)$ of $\mathrm{C}_{60}$ and use its sensitivity to isotopic substitution as a way to discriminate SingleMolecule $\mathrm{C}_{60}$ events. Silver nanoparticle aggregates and $\mathrm{C}_{60}$ were deposited on a planar substrate and SERS spectral maps were recorded. We used linear fits of the series of SERS spectra of $\mathrm{C}_{60}$ isotopologues in the $A_{g}(2)$ region. We were expecting the central frequency of the $A_{g}(2)$ mode to span a range of $\sim 20 \mathrm{~cm}^{-1}$ (see Sec. 5.5.2) so we adapted the linear least-squares fitting routine as follows:

* Instead of using the average spectrum of the maps as the reference, we used a Lorentzian function centered in $x_{0 \text { ref. }}$. The background was also set to be quadratic so $\vec{\alpha}$ was of length 4 .

* Then, for each spectrum, the central frequency of the Raman peak is allowed to be different from the one of the reference by testing the goodness of the fit for 40 different positions of the central frequency of the reference corresponding to 20 shifts left of 1 pixel and 20 shifts right of 1 pixel. After the 40 iterations, the best fit (i.e. the position giving the smallest sum of squared residuals) is stored.

* Finally, different FWHM for the reference are tested. This is done by repeating the previous step for $\Gamma=1 . .10$ and storing the final parameters vector $\vec{\alpha}$ for which the overall smallest sum of leastsquared residues is obtained.

Hence, for each of the 15000 spectra of the series of experimental data, 400 linear least-squared fits were performed so a total of 6 million fits; yet it was a lot faster than non-linear least-squares Voigt fits and the discrimination of SM-events was relatively straightforward then; they were the events for which $\alpha_{1}$ was not negligible and $\Gamma$ was around 4 to $5 \mathrm{~cm}^{-1}$. Bigger $\Gamma$ correspond to multi- $\mathrm{C}_{60}$ molecule events. 


\section{Chapter 4}

\section{Measurement of the}

\section{homogeneous broadening of spontaneous resonant Raman lines via SM-SERS}

\subsection{Introduction}

A basic concept in spectroscopy (Raman in particular) is that of homogeneous / inhomogeneous broadening of features [58]. While the former has its origin in intramolecular anharmonic interactions, and is a fundamental property of a vibration of an isolated molecule, the latter is representative of a population of molecules and their inevitable variabilities at different places in the sample. The inhomogeneous broadening can be a substantial contribution to the actual measured peak width, especially if its intrinsic linewidth is comparable to the shifts in frequency induced by external interaction between the molecules and their environment or isotopic variations as we will see in Chapter 5. A conventional Raman experiment involves the monitoring of (at least) $10^{4}$ to $10^{6}$ molecules in the scattering volume, thus the intrinsic homogeneous broadening of the peaks is usually convoluted with the inhomogeneous broadening. If one wants to access the homogeneous broadening of a peak, one has to mea- 
sure individual molecules; this is the motivation of the study presented in this chapter. By using SM-SERS, one can disentangle the contributions to the inhomogeneous broadening, and access the intrinsic homogeneous broadening of a molecule on a surface.

\subsection{Homogeneous and inhomogeneous broad- ening in Raman spectroscopy}

The notions of lifetime of an excited quantum state or the broadening associated with a transition between two quantum states, are central in spectroscopy, whether electronic (UV-vis absorption), vibrational (Raman) or spin (NMR) transitions are studied. But the terminology which is used tends to vary quite a lot depending on the community which can lead to a lot of confusion. The finite lifetime (energy relaxation) or dephasing of the excited states is the reason why energy levels are intrinsically broadened [58]; this is what is usually referred to as homogeneous broadening. There are further mechanisms of broadening, on top of the homogeneous one, which are referred to as inhomogeneous as they depend on a particular emitter of a species and its environment or are limitations of the detection technique such as the spectral resolution limit. The following section gives a brief overview of the different terms and mechanisms they encompass in order to set the context of the problematic of this chapter.

\subsubsection{Homogeneous broadening of a molecular vi- bration}

\section{Homogeneous broadening: definition}

Homogeneous broadening is usually referred to as the intrinsic effects which increase the linewidth of a vibrational transition of a single molecule in a particular environment at a given temperature $\mathrm{T}$ [58]. For example, a single molecule will have transitions exhibiting a natural linewidth due to the finite lifetime and coherence time of the excited levels. We 
will in this thesis refer to the linewidth of a vibration as being homogeneous when the spectrum comes from a single molecule and is hence not a superposition of peaks with slightly different positions (inhomogeneous broadening).

The broadening of the vibrational energy levels has its origin in the anharmonicity of the vibrations and is not predicted by the harmonic approximation [59]. The harmonic approximation consists in expanding the potential energy of the nuclei of a molecule in powers of the atomic displacements and terminating the expansion at the term quadratic in displacement (see Sec. 5.4.2). The most significant result from this approximation is the possibility of decoupling the molecular vibrations by a transformation to normal coordinates, leading to the concept of phonons or normal modes as non-interacting quantized vibrational excitations. When we take into account higher terms in the expansion of the potential energy (order 3 and higher terms), the decoupling is no longer perfect, and phonon-phonon interactions terms appear [59]. As a result of interactions with other intramolecular modes or intermolecular modes or modes available in the environment, a vibrational mode will eventually disappear after a finite time and the frequencies of the normal modes one get in the harmonic approximation are shifted when anharmonic interactions are introduced. Anharmonic interactions play a fundamental role in the relaxation pathways of vibrations towards equilibrium [60]. Intramolecular anharmonic interactions tend to dominate the relaxation pathways of vibrations for many molecules [61], but interactions with the substrate or the solvent can provide additional pathways that dominate the relaxation in other cases. The finite lifetime of the vibrations shows itself in a spectrum by the broadening of the lines.

One can distinguish two types of interactions: interactions resulting in a loss of population of the vibration or energy relaxation and interactions resulting in pure dephasing of the vibration, which cause fluctuations of the transition frequency without level decay (without loss in energy). The linewidth $\Gamma$ (in $\mathrm{cm}^{-1}$ ) of an homogeneously broadened transition has 
then two contributions:

$$
\Gamma\left[\mathrm{cm}^{-1}\right]=\Gamma_{1}+\Gamma_{2}^{\prime}=\frac{1}{2 \pi c}\left(\frac{1}{T_{1}}+\frac{2}{T_{2}^{\prime}}\right)
$$

where $2 T_{1}$ is the population decay time associated with energy relaxation (population amplitude decay) of the excited level and $T_{2}^{\prime}$ is the dephasing time, associated with the loss of coherence of the vibration. The factor 2 comes from the density matrix formalism where the density matrix $\hat{\rho}$ is a tool to represent the state of a quantum system which can be in a statistical mixture of states (a molecule coupled to a bath for example). The off-diagonal terms are called coherences and are associated with pure dephasing which is a property of the probability amplitude. The diagonal terms are called the populations and are connected to the square of the probability amplitude.

Much work has been done in the 1970s and 1980s towards an improved understanding of what determines the observed vibrational linewidth of molecules, whether they are in liquid phase, crystalline phase, at metallic surfaces or non-metal surfaces. And for each scenario, what is the relative importance of population and pure phase relaxation ?[60, 61, 62, $63,64,65]$. And in the case of $T_{1}$ processes dominating the linewidth, what mechanisms lead to the relaxation of level populations: damping via the formation of electron-hole pairs, creation of metal phonons or either intramolecular or intermolecular vibrational energy transfer (VEE, [66])? Time-resolved optical spectroscopic techniques $[67,68]$ have the advantage of being able to resolve separately population relaxation and pure dephasing by using a variety of time delayed pulse sequences. Conversely, with frequency-resolved spectroscopies, such as CW Raman spectroscopy, these quantities are only accessible by lineshape analysis and their disentanglement is not straightforward.

\section{Simple illustration of homogeneous broadening mechanisms}

Let us describe an excited vibration of a molecule $q(t)$ by the classical model of an harmonic oscillator with angular frequency $\omega_{0}$. If this harmonic vibration has an infinite lifetime and undergoes no dephasing, then 
the intensity distribution in the frequency domain is a Dirac peak at $\omega_{0}$ and hence exhibits no broadening.

Let us now examine the case of a damped harmonic oscillator; the energy loss results in a damping of the oscillation described by the damping constant $\Gamma$ :

$$
q(t)=e^{-\frac{\Gamma}{2} t} \cos \left(\omega_{0} t\right)
$$

This vibration with central frequency $\omega_{0}$ can be written as a superposition of harmonic oscillations with slightly different frequencies $\omega$ and amplitudes $A(\omega)$ :

$$
q(t)=\Re\left(\frac{1}{2 \sqrt{2 \pi}} \int_{0}^{\infty} A(\omega) e^{i \omega t} d \omega\right)
$$

$A(\omega)$ is the frequency distribution and is then related to $q(t)$ by Fourier transform. The intensity profile $I(\omega)$ is proportional to $|A(\omega)|^{2}$. Within the classical model of the damped harmonic oscillator,

$$
\begin{aligned}
A(\omega) & =\frac{1}{2 \sqrt{2 \pi}} \int_{-\infty}^{+\infty} q(t) e^{-i \omega t} d t \\
& =\frac{1}{2 \sqrt{2 \pi}} \int_{0}^{+\infty} q_{0} e^{-(\Gamma / 2) t} \cos \left(\omega_{0} t\right) e^{-i \omega t} d t \\
& =\frac{q_{0}}{2 \sqrt{2 \pi}}\left(\frac{1}{i\left(\omega-\omega_{0}\right)+\Gamma / 2}+\frac{1}{i\left(\omega+\omega_{0}\right)+\Gamma / 2}\right)
\end{aligned}
$$

It follows:

$$
I(\omega) \propto A^{*}(\omega) A(\omega) \approx \frac{C}{\left(\omega-\omega_{0}\right)^{2}+(\Gamma / 2)^{2}}
$$

when $\omega$ is close to $\omega_{0}$. This intensity profile is a Lorentzian function (see Fig. 4.1 b)). After normalization, the real intensity profile is:

$$
I\left(\omega-\omega_{0}\right)=I_{0} \frac{(\Gamma / 2)^{2}}{\left(\omega-\omega_{0}\right)^{2}+(\Gamma / 2)^{2}}
$$


where $I_{0}=I\left(\omega=\omega_{0}\right)$. The Full Width at Half Maximum (FWHM) is $\Gamma$. When $\Gamma$ is given in $\mathrm{cm}^{-1}$, it is related to the lifetime $T_{1}$ of the vibration by:

$$
\Gamma=\frac{1}{2 \pi c} \cdot \frac{1}{T_{1}}
$$

Figure 4.1 illustrates the broadening of the spectral lines which is induced by the exponential decay of the damped harmonic oscillator $q(t)$.

Let us now consider the case where an harmonic oscillation experiences pure dephasing. The decay of correlations in the oscillator coordinate $q(t)$ results from random phase factors acquired in elastic collisions with the surroundings. We can illustrate this mechanism with the oscillator $q(t)$ propagating in an undamped manner during a time $2 \tau_{c}$ and then undergoing a phase shift $\delta$. The coordinate outside the window $2 \tau_{c}$ is uncorrelated to the coordinate within due to the acquisition of the random phase factor. Let us write:

$$
q(t)=\left\{\begin{array}{lll}
\Re\left(q_{0} e^{i \omega_{0} t}\right) & \text { for } & |t|<\tau_{c} \\
\Re\left(q_{0} e^{i \omega_{0} t+\delta}\right) & \text { for } & |t| \geq \tau_{c}
\end{array}\right.
$$

One can show [65] that in this case, the intensity profile, which is obtained by taking the Fourier transform of the correlation function of $q(t)$, has the lineshape in the frequency domain:

$$
I(\omega)=\frac{2 \sin ^{2}\left[\left(\omega-\omega_{0}\right) \tau_{c}\right]}{\tau_{c}\left(\omega-\omega_{0}\right)^{2}}
$$

The appearance of the factor $\sin ^{2}\left[\left(\omega-\omega_{0}\right) \tau_{c}\right]$ is a consequence of the correlation function having an envelope with sharp "corners" (triangular envelope, consequence itself of the collision model and sudden jump in phase). If the envelope of the correlation function was exponential, a pure Lorentzian lineshape would be retrieved for $I(\omega)$ which corresponds to what is observed experimentally.

In Fig. 4.2 a), we illustrate numerically the broadening induced by 


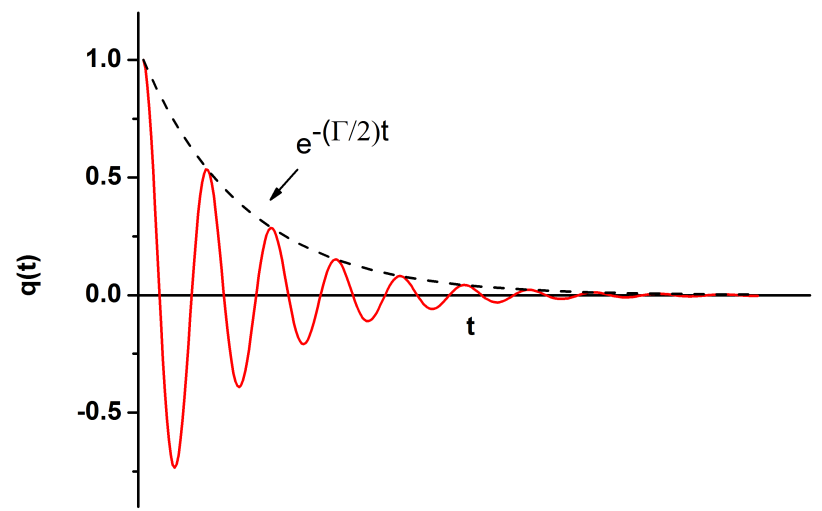

(a) Damped oscillator trajectory: $q(t)=e^{-(\Gamma / 2) t} \sin \left(\omega_{0} t\right)$

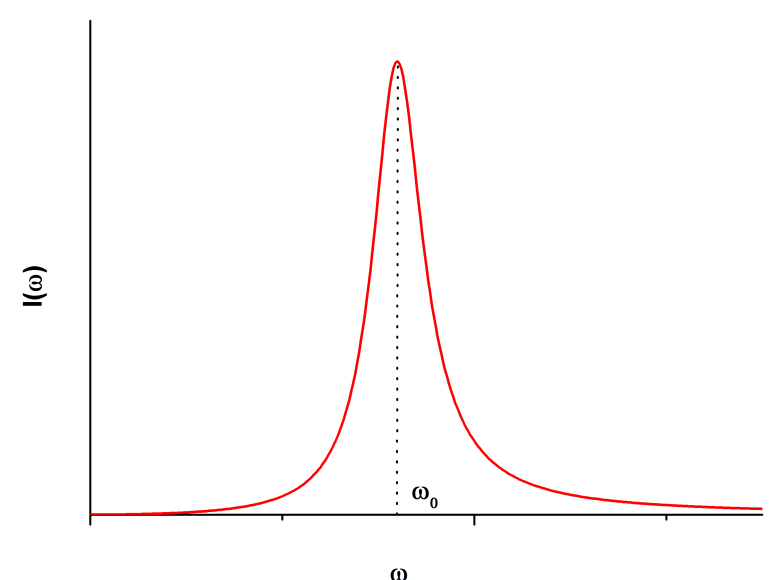

(b) $I(\omega)$ obtained by Fast Fourier Transform.

Figure 4.1: Numerical illustration of the broadening of the spectral lines induced by damping. The lineshape in b) is a Lorentzian function whose FWHM is $\Gamma$. 


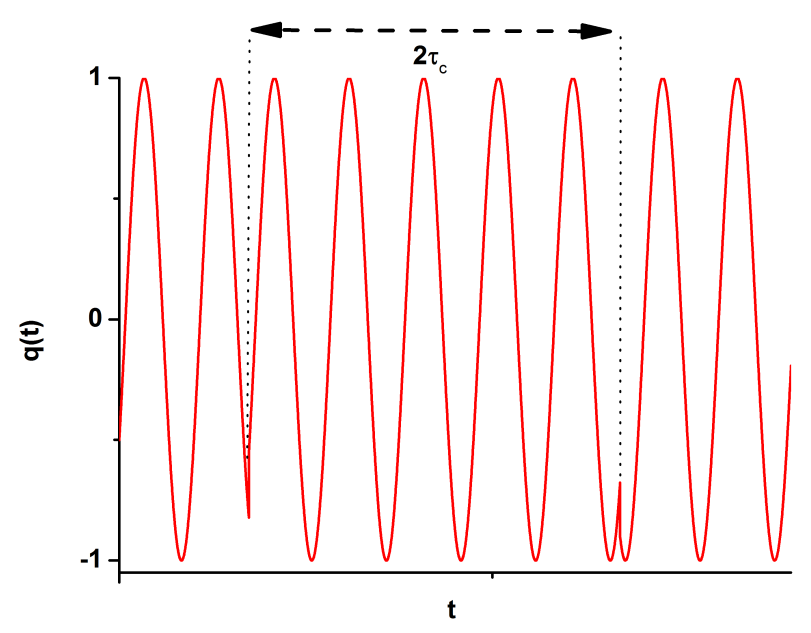

(a) Oscillator trajectory: $q(t)=\sin (t+\operatorname{rand}(1) \times 2 \pi)$

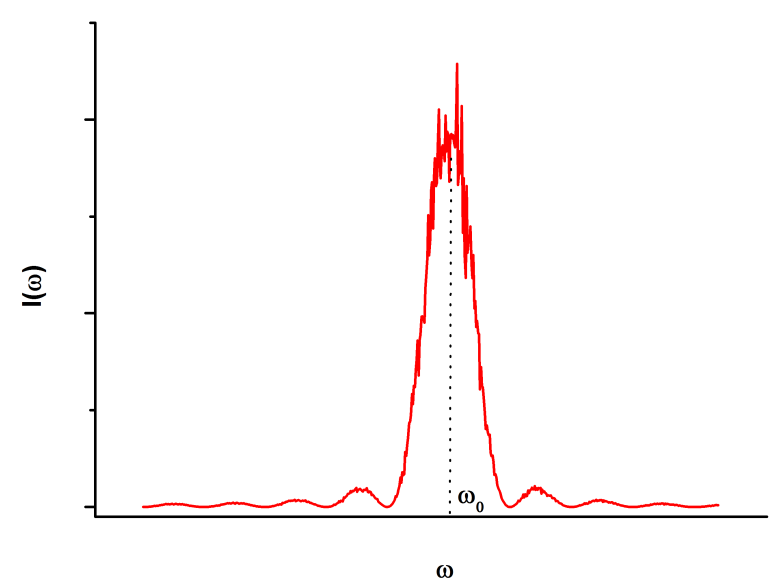

(b) $I(\omega)$ obtained by fft.

Figure 4.2: Numerical illustration of the broadened spectral lines induced by loss of correlation. We can see that $I(\omega) \equiv \operatorname{sinc}^{2}\left(\omega-\omega_{0}\right)$. The FWHM of b) is $\frac{1}{2 \tau_{c}}$. 
pure dephasing by $q(t)$ being a sine curve over [-1000, 1000] undergoing 64 consecutive shifts in phase of a random fraction of $2 \pi$. These jumps in phase occur at regular intervals $2 \tau_{c}$. The corresponding spectral distribution in Fig. 4.2 b) was obtained by generating 100 different functions $q(t)$, taking their Fourier transform with the Fast Fourier Transform (FFT) in Matlab and taking the average. One can readily see that the lineshape which is obtained this way is that of a squared cardinal sine function (sinc), as expected.

\subsubsection{Instrumental broadening}

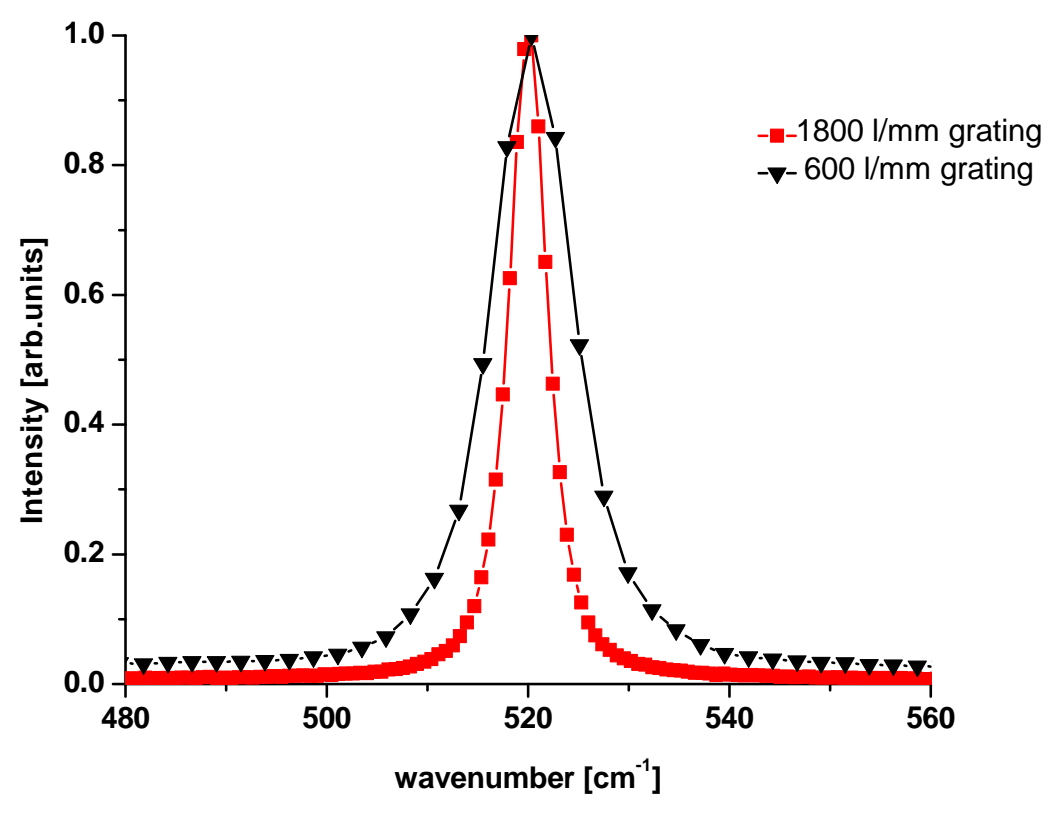

Figure 4.3: Example of instrumental broadening: broadening induced by the use of a lower resolution grating. Raman spectrum of silicon [100] around the $520 \mathrm{~cm}^{-1}$ mode taken at $458 \mathrm{~nm}$ excitation, $3 \mathrm{~s}$ acquisition, with the $600 \mathrm{l} / \mathrm{mm}$ grating (black) and the $1800 \mathrm{l} / \mathrm{mm}$ grating (red).

What we detect in a conventional spectroscopy experiment is usually not the pure Lorentzian line profile with natural linewidth $\Gamma$ as the latter is concealed by other broadening effects such as instrumental broadening and inhomogeneous broadening. 
The response function of the spectrometer can introduce instrumental broadening; it comes from the finite resolution of the diffraction gratings and the CCD. In our Raman experiment, the spectral resolution is determined by: the incident laser wavelength (the higher the wavelength, the better the resolution), the number of grooves (lines) per millimeter, and the size of the image of the sample on the entrance slit (the resolution deteriorates as the size of the image on the entrance slit increases). If the image is small, ideally a "point-like" source, the position of the peak will still depend on the location of the image with respect to the optical axis. By reducing the size of the entrance slit, one can remove the contributions to the signal coming from point-sources with too much departure from the axis and hence increase the resolution; this procedure ensures that any variation in frequency of the peak will be due to inhomogeneous broadening (see below) and not to image location. Figure 4.3 shows the influence of the choice of gratings on the resolution and thus the measured width of the Raman peak of silicon at $520 \mathrm{~cm}^{-1}$.

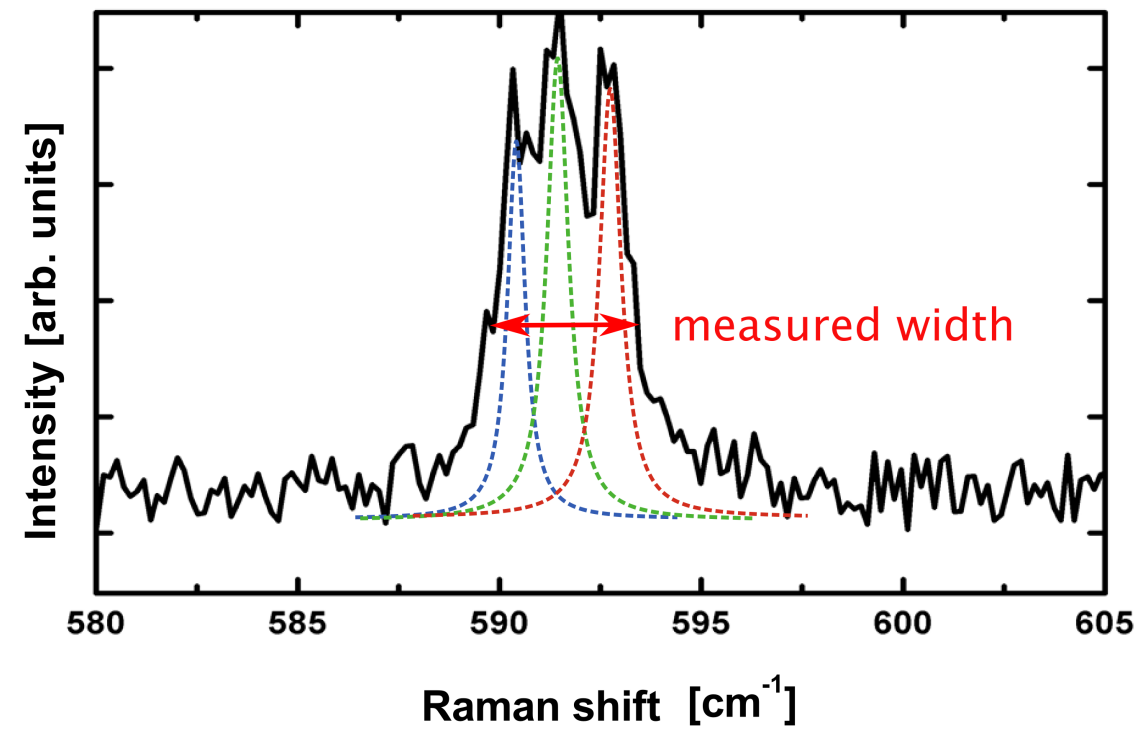

Figure 4.4: Example of inhomogeneous broadening of a peak due to the simultaneous measurement of three molecules having their central frequency slightly different from each other. The data are from the $590 \mathrm{~cm}^{-1}$ mode of Nile blue taken at $80 \mathrm{~K}$ at $633 \mathrm{~nm}$ with the high resolution $2400 \mathrm{l} / \mathrm{mm}$ grating. 


\subsubsection{Mechanisms of inhomogeneous broadening}

In Section 4.2.1, we have defined the homogeneous broadening as a broadening of a single oscillator or a single molecule. Conversely, we will define in this work the inhomogeneous broadening of a Raman peak as the broadening which comes from the fact that the signal arises from a population of molecules which can have each slightly different interactions with the surrounding medium (which lead to slightly different frequency positions for a given peak and possibly slightly different widths). Additional contributions to the inhomogeneous broadening can also come from the presence of isotopologues in a given population as we will see is the case for the $A_{g}(2)$ mode of $\mathrm{C}_{60}$ in Chapter 5 . The concept of inhomogeneous broadening underlies entire laser spectroscopy techniques [58] (like 'hole-burning' for example). In spectroscopy in liquids, solvation effects (intermolecular interactions) are formally considered to be part of the inhomogeneous broadening processes. But in the case of SERS, the environment is the SERS substrate itself and additional variations in the conditions of the molecules may come from interactions with it. Figure 4.4 shows the effects of frequency variations of the inhomogeneous broadening of a measured peak. In Refs. [22, 51], a systematic study of the origin of the inhomogeneous broadening in SM-SERS spectra (and the origin of frequency variations) was carried out.

Hence, at a formal level, we can say that the detection of a peak in a spectrometer is a triple convolution problem; the homogeneous broadening is convoluted with the inhomogeneous contributions to the linewidth and this is further convoluted with the response function of the spectrometer.

\subsubsection{Anharmonic coupling of phonons and Vibra- tional Energy Exchange Model (VEE)}

The temperature $(T)$ dependence of the homogeneous width of a Raman mode provides information on the anharmonic coupling of that particular mode with the rest of the vibrations and/or the substrate [69].

The lowest order anharmonic interactions (cubic term of the Hamil- 


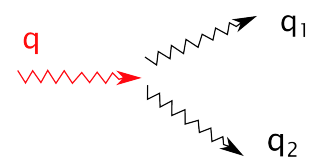

$\mathrm{q}_{1}$

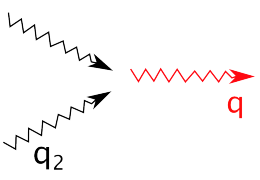

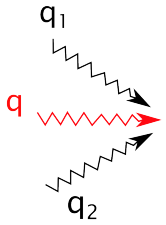

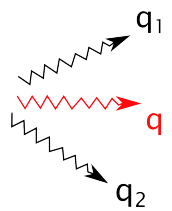

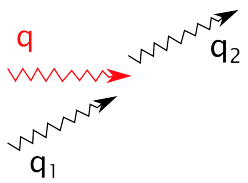

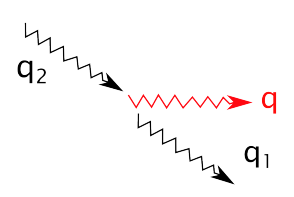

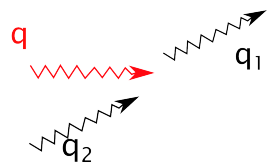

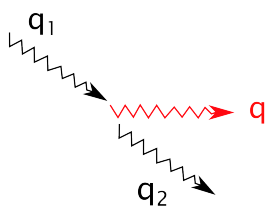

Figure 4.5: Feynmann diagrams for the three-phonon interaction processes. In the first row, the phonon $\vec{q}$ is annihilated and in the second row, the phonon $\vec{q}$ is created. These processes are described by the cubic term of the Hamiltonian, which was the first left out in the harmonic approximation.

tonian) for a phonon $\vec{q}$ of frequency $\omega_{q}$ are shown in Fig 4.5: they correspond to three-phonon interactions. The two processes corresponding to the simultaneous creation or annihilation of three phonons violate energy conservation but they are possible virtual intermediate subprocesses of multi-step interactions [59]. In three-phonon interactions, the number of phonons changes. The decay route which corresponds to the first row of Fig 4.5 results in the decay of the original phonon $\vec{q}$ and hence is the main (however not sole) contribution to $T_{1}$ processes (population decay). The created phonons $\overrightarrow{q_{1}}$ and $\overrightarrow{q_{2}}$ can be intramolecular modes of the molecule or available vibrations in the environment. The rate at which the phonon $\vec{q}$ disappears as a result of interactions with $\overrightarrow{q_{1}}$ and $\overrightarrow{q_{2}}$ is the sum of the probabilities of processes in the first row minus the sum of the probabilities of the processes of the second row. These probabilities directly depend on the occupation number $n_{1}$ and $n_{2}$ in modes $\overrightarrow{q_{1}}$ and $\overrightarrow{q_{2}}$. Phonons are bosons hence $n_{1}$ and $n_{2}$ can be expressed at thermal equilibrium by the Bose-Einstein distribution:

$$
\left\langle n_{i}\right\rangle=\frac{1}{\exp \left(\frac{\hbar \omega_{i}}{k_{B} T}\right)-1}
$$

where $\left\langle n_{i}\right\rangle$ is the average number of phonons of angular frequency $\omega_{i}$ 
or phonon occupancy of the mode $\omega_{i}$. At low temperatures, Eq. 4.10 becomes:

$$
\left\langle n_{i}\right\rangle \sim \exp \left(-\frac{\hbar \omega_{i}}{k_{B} T}\right)
$$

Hence one can see that the rate at which the phonon $\vec{q}$ disappears, thus its lifetime $\left(T_{1}\right)$ will be dependent on the temperature through its anharmonic interactions with other phonons. The temperature dependence of the peak width of the phonon due to population relaxation follows an Arrhenius type law:

$$
\Gamma=\Gamma_{0}+\Delta \Gamma \exp \left(\frac{-\hbar \Omega}{k_{B} T}\right)
$$

where $\Omega$ is the frequency of the boson/vibration to which the system is coupled. This has been shown to be true even when one takes into account higher order multi-phonon decay processes [70, 71], which are weaker but can contribute nevertheless to the broadening. The vibrational energy of the molecule indeed includes contributions from morethan-three phonon processes like four-phonon processes and higher. Multiphonon relaxation is not a probable decay route for the polyatomic molecules we will consider in this Chapter but it can become a dominant mechanism for population relaxation when a single isolated vibration has a much higher energy than the remaining low frequency vibrations in the molecule or in the continuum provided by the environment (solvent, substrate), also called bath or reservoir [72].

There are also three-phonon processes for which the phonon distribution is the same in the initial and final states. They consist of two-step processes, in each case the first process, leading to the virtual intermediate step, is identical with one of the eight processes of Fig. 4.5. Fig. 4.6 shows two examples of these 2-step processes involving the three phonons $\vec{q}, \overrightarrow{q_{1}}$ and $\overrightarrow{q_{2}}$. Two examples of these processes are shown in Fig. 4.6. Because the molecule returns to its original state, these processes do not contribute to $T_{1}$ (loss of population) but instead, contribute to the broadening of the spectral line via $T_{2}^{\prime}$ (pure dephasing) [73]. 

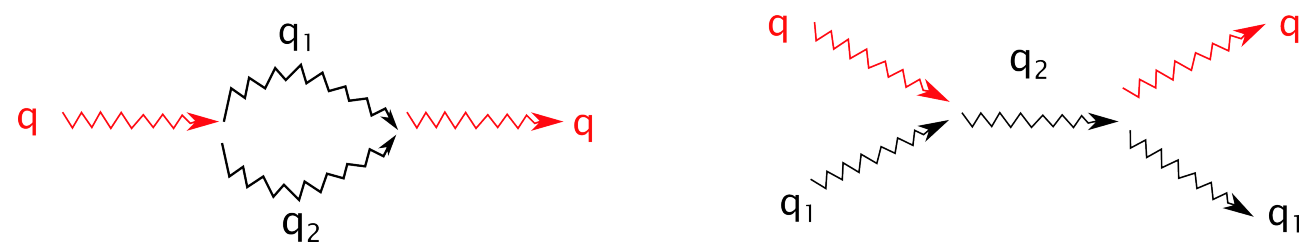

Figure 4.6: Feynmann diagrams for two examples of two-step phononphonon interactions. These processes contribute to the spectral broadening mainly via pure dephasing.

The broadening of a mode due to pure dephasing through anharmonic coupling between phonons is also temperature dependent and also takes on an Arrhenius-like form. Some approaches to this problem are presented in $[72,74,75]$. The following section presents a model for dephasing of a high frequency mode caused by intramolecular anharmonic coupling to a low-frequency vibration which is an extension of the exchange model of Anderson and Kubo [76, 77].

\section{Vibrational energy exchange model for the temperature depen- dence of the spectral homogeneous broadening of a vibration}

When exploring the dephasing processes for vibrational relaxation, several models $[78,79]$ have been advanced to relate the experiments to the specific interactions responsible for dephasing. These models treat the system as a harmonic oscillator weakly coupled to a reservoir of oscillators in thermal equilibrium. In the case of polyatomic molecules however this is complicated by the fact that some intramolecular modes may interact more strongly with the vibration of interest than does the bath. In their article [80], Harris et al. show that the Raman vibrational dephasing times can reflect random frequency modulation of high-frequency modes via exchange with low-frequency modes and that the temperature dependence of the spontaneous Raman spectrum (lineshape and Raman shift) can reveal quantitatively lifetimes and scattering rates between normal modes.

The idea is as follows (se Fig. 4.7):

1. The degrees of freedom of the system $\{$ molecule + environment $\}$ 


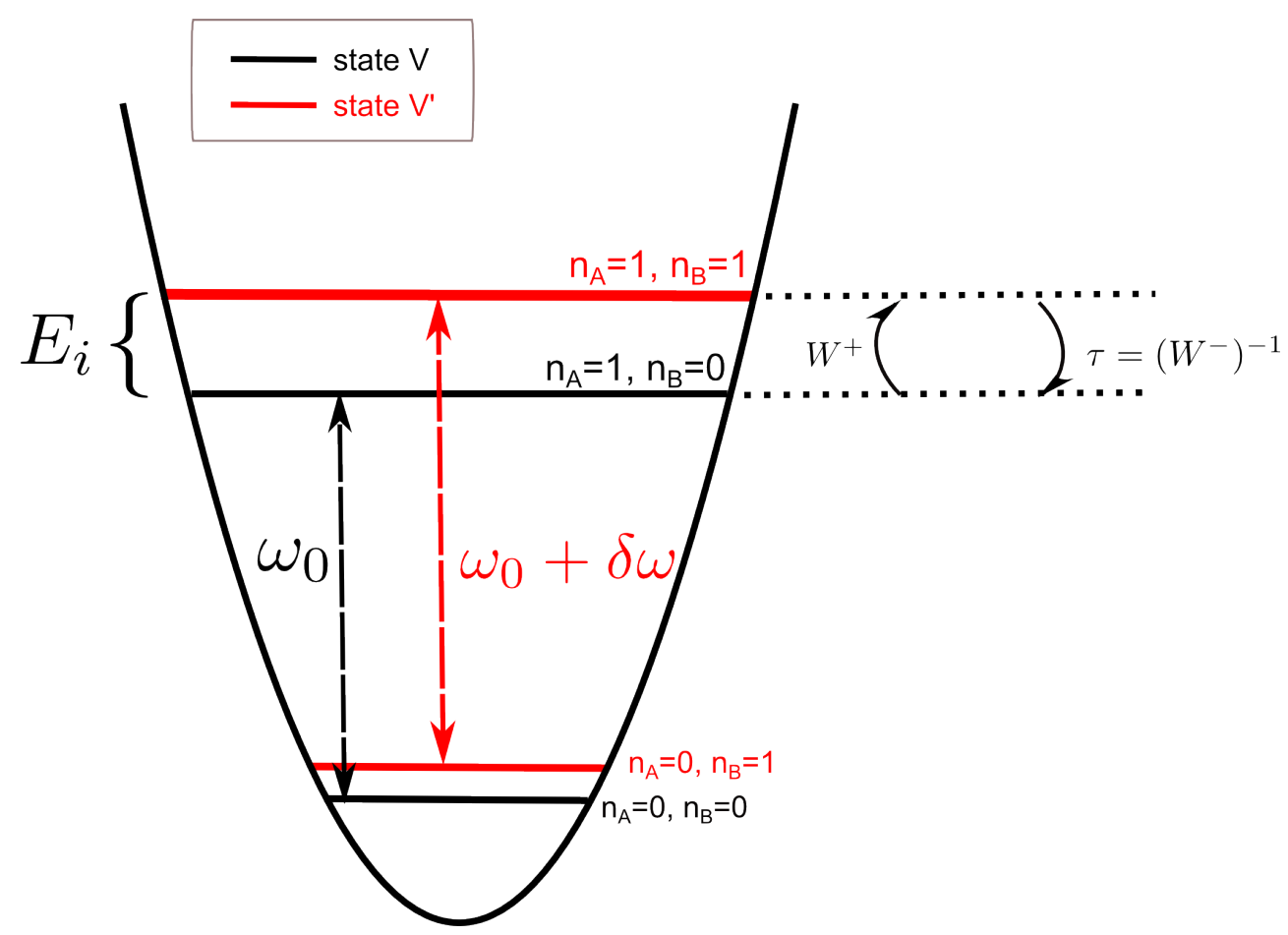

Figure 4.7: Energy levels for vibrational exchange between the states $V$ and $V^{\prime}$. The vibrational mode $\mathrm{A}$ with frequency $\omega_{0}$ and occupancy $n_{A}$ interacts with a low frequency mode $\mathrm{B}$ (occupancy $n_{B}$ ) such that the excitation of the low-frequency mode shifts the frequency of A by $\delta \omega$. $W^{+}$is the scattering rate from $V$ and $\tau$ is the true lifetime in $V^{\prime}$. 
are partitioned into three groups: the vibrational mode A (quantum number $n_{A}$, frequency $\omega_{0}$ ) whose dephasing is under study, the exchanging modes and the reservoir. For simplification we assume that, for a given temperature range, one exchange mode B (quantum number $n_{B}$ ) only is responsible for scattering of phonons to higher energy states. We separate relaxation to other states $\left(T_{1}\right.$ channels) from scattering between the initial state $V$ and the other vibrational state $V^{\prime}$. The energy of the state $V^{\prime}$ is at an energy $E_{i}$ above the initial state (See Fig. 4.7).

2. When the anharmonic perturbations which couple these 2 modes are taken into account, the levels shift such that a number of vibrational frequencies are possible for the high frequency mode A, depending on the occupation of the low frequency mode B. Excitation and relaxation (population relaxation) of the low frequency mode can now cause dephasing of the high frequency mode through modulation of its vibrational frequency.

3. When the rates from vibrational energy exchange are incorporated, we get the expressions for the effective vibrational frequency $\omega_{\text {eff }}$ and overall relaxation time $T_{\text {eff }}$ for the mode $\mathrm{A}$ under study [80]:

$$
\begin{aligned}
& \omega_{\text {eff }}=\omega_{0}+\frac{\delta \omega W^{+} \tau}{1+(\delta \omega)^{2} \tau^{2}} \\
& \left(T_{\text {eff }}\right)^{-1}=T_{1}^{-1}+T_{2}^{\prime-1}+W^{+} \frac{(\delta \omega)^{2} \tau^{2}}{1+(\delta \omega)^{2} \tau^{2}}
\end{aligned}
$$

where $W^{+}$is the rate of scattering from state $V$ to $V^{\prime}$ and $\tau$ is the true lifetime (population relaxation time) in $V^{\prime}$ which is then not obscured by contributions from dephasing.

4. As $\tau$ can be considered to be independent of temperature, the principle of detailed balance states that $W^{+}$will be expected to depend on the temperature $T$ as $W^{+}=\tau^{-1} \exp \left(-E_{i} / k_{B} T\right)$. This leads to 
the observed temperature dependence:

$$
\begin{gathered}
\omega_{\text {eff }}=\omega_{0}+\frac{\delta \omega \tau}{1+(\delta \omega \tau)^{2}} \frac{1}{2 \pi c \tau} \exp \left(-E_{i} / k_{B} T\right) \\
\left(T_{\text {eff }}\right)^{-1}=\frac{(\delta \omega \tau)^{2}}{1+(\delta \omega \tau)^{2}} \frac{1}{\pi c \tau} \exp \left(-E_{i} / k_{B} T\right)
\end{gathered}
$$

where $\omega_{\text {eff }}$ and $\left(T_{\text {eff }}\right)^{-1}$ are expressed in $\mathrm{cm}^{-1}$.

The temperature dependent frequency shift together with a similar temperature dependent homogeneous broadening is the signature of vibrational exchange between two or more normal modes, and when both of them are analyzed, one obtains $W^{+}, \delta \omega$ and $\tau$ which are not quantities easily accessible otherwise.

\subsection{Temperature dependence of the homo- geneous broadening of the $590 \mathrm{~cm}^{-1}$ mode of Nile blue}

\subsubsection{Immediate context of the study}

The work published by our group in 2010 in [51] has shown that it is possible to resolve single molecule contributions within the inhomogeneous broadening of peaks: the proof of concept was made using the $590 \mathrm{~cm}^{-1}$ mode of Nile blue and the $2226 \mathrm{~cm}^{-1}$ mode of rhodamine 800 at low temperature $(80 \mathrm{~K})$. The origin of inhomogeneous broadening in SERS spectra was systematically studied, so was the origin of frequency variations between different events [22]. Moreover, an important application of SM-SERS to disentangle the inhomogeneous contributions in the Raman spectrum has been demonstrated in [16] where it is shown that single molecule surface enhanced Raman excitation profiles are narrow in comparison to the ensemble-averaged excitation profiles.

Having the tools to measure confidently individual molecules with SERS with a high spectral resolution, it is natural to investigate whether one could observe a significant temperature dependence of the linewidth 
of the single molecule events and, in that case, what could be learned about the vibrational relaxation pathways of an excited vibration of a molecule adsorbed onto a metallic surface in SERS conditions. The study of the lineshape and linewidth of vibrations at the single molecule level in the frequency domain by direct CW Raman measurement is now accessible, free from inhomogeneous contributions.

\subsubsection{Experiments}

SM-SERS experiments were carried out for the dye Nile blue (NB) excited resonantly at $633 \mathrm{~nm}$, focusing, in particular, on the $590 \mathrm{~cm}^{-1}$ Raman mode. The conditions under which SM-SERS can be observed in this system have been previously studied in detail [51]. Briefly, a mixture of either citrate- reduced Lee \& Meisel or borohydrate reduced Ag colloids [48], $\mathrm{KCl}$ and Nile blue (final concentration $5 \mathrm{nM}$ ) was drop-cast on polyL-lysine coated Si wafers then siphoned off, as described in Chapter 2.

Raman spectra were acquired using the $\times 100$ LWD objective $(\mathrm{NA}=$ 0.6) with excitation at $633 \mathrm{~nm}$ and an incident power of $2.5 \mathrm{~mW}$ on the sample. In order to minimize the instrumental broadening, we used the high resolution $2400 \mathrm{l} / \mathrm{mm}$ grating. At this wavelength, this grating allows us to achieve a resolution of $\sim 0.17 \mathrm{~cm}^{-1}$ per pixel thus enabling the distinction of small frequency variations as well as individual contributions from more than one molecule to the linewidth.

The temperature was varied between $80 \mathrm{~K}$ to room temperature with the Linkam-Scientific THMS600 stage for optical microscopy, which keeps the sample under a $\mathrm{N}_{2}$ atmosphere. SM-SERS spectra were obtained from several spatial maps of $30 \times 30$ scans with varying steps $(1$ to $5 \mu \mathrm{m})$ and $1 \mathrm{~s}$ integration time per point. 3600 spectra were then recorded for each temperature: $80 \mathrm{~K}, 98 \mathrm{~K}, 123 \mathrm{~K}, 198 \mathrm{~K}$ and $298 \mathrm{~K}$. Each spectrum was fitted with a pseudo-Voigt lineshape (see Chapter 3). A large number of low intensity events (below noise level) are then discarded, as they correspond to locations with no signal. This large amount of "waste" is unavoidable to ensure the single molecule nature of the remaining observable spectra. 


\subsubsection{Results and discussion}

Figure 4.8 (a-e) shows a representative collection of some of the narrowest SERS spectra of the data at different temperatures. A quick qualitative analysis showed first that, in the data, the spectra showing signal in the $590 \mathrm{~cm}^{-1}$ region were of two types: very narrow events which have varying frequency positions and broaden with increasing temperature and broader events exhibiting no significant frequency variations nor significant increase in the broadening with higher temperature: we assign these narrow events to SM-SERS events and the wider events to manymolecules SERS events (see below). The frequency variations can be easily appreciated in Fig. 4.8 together with the change in the intrinsic broadening of the peaks. Average spectra are also shown in Fig. 4.8 (f) for $80 \mathrm{~K}$ and $298 \mathrm{~K}$. Clearly, the frequency variations is the dominant contribution to the inhomogeneous broadening (i.e. that of the average). It is worth noticing at this point that, at $80 \mathrm{~K}$, the single NB events for the $590 \mathrm{~cm}^{-1}$ mode are quite narrow $\left(\sim 0.85 \mathrm{~cm}^{-1}\right)$ compared to the average signal taken at the same temperature $\left(\sim 3.5 \mathrm{~cm}^{-1}\right)$, this suggests that more than one molecule can be resolved within the inhomogeneous broadening of the peak.

We carried out a more detailed statistical analysis based on non-linear fits to pseudo-Voigt profiles (see Chapter 3) to discriminate "narrow" events from average, wide events and hence sort SM-events and many molecule events, then further reveal the temperature dependence of both homogeneous broadening and frequency shifts, as was suspected by the qualitative analysis of the data. To this end, we used the full-widths at half-maximum (FWHM) $\Gamma$ obtained from the fits to discriminate SMevents.

Figure 4.9 summarizes the results of the pseudo-Voigt fits. Lowest intensity events (below noise level) have been removed from the statistics, and for each temperature, only the most intense and narrowest events have been included in the graph. One can appreciate that the intensity of these events varies greatly; which reflects the distribution of enhancement factors over several orders of magnitude that one typically obtains 


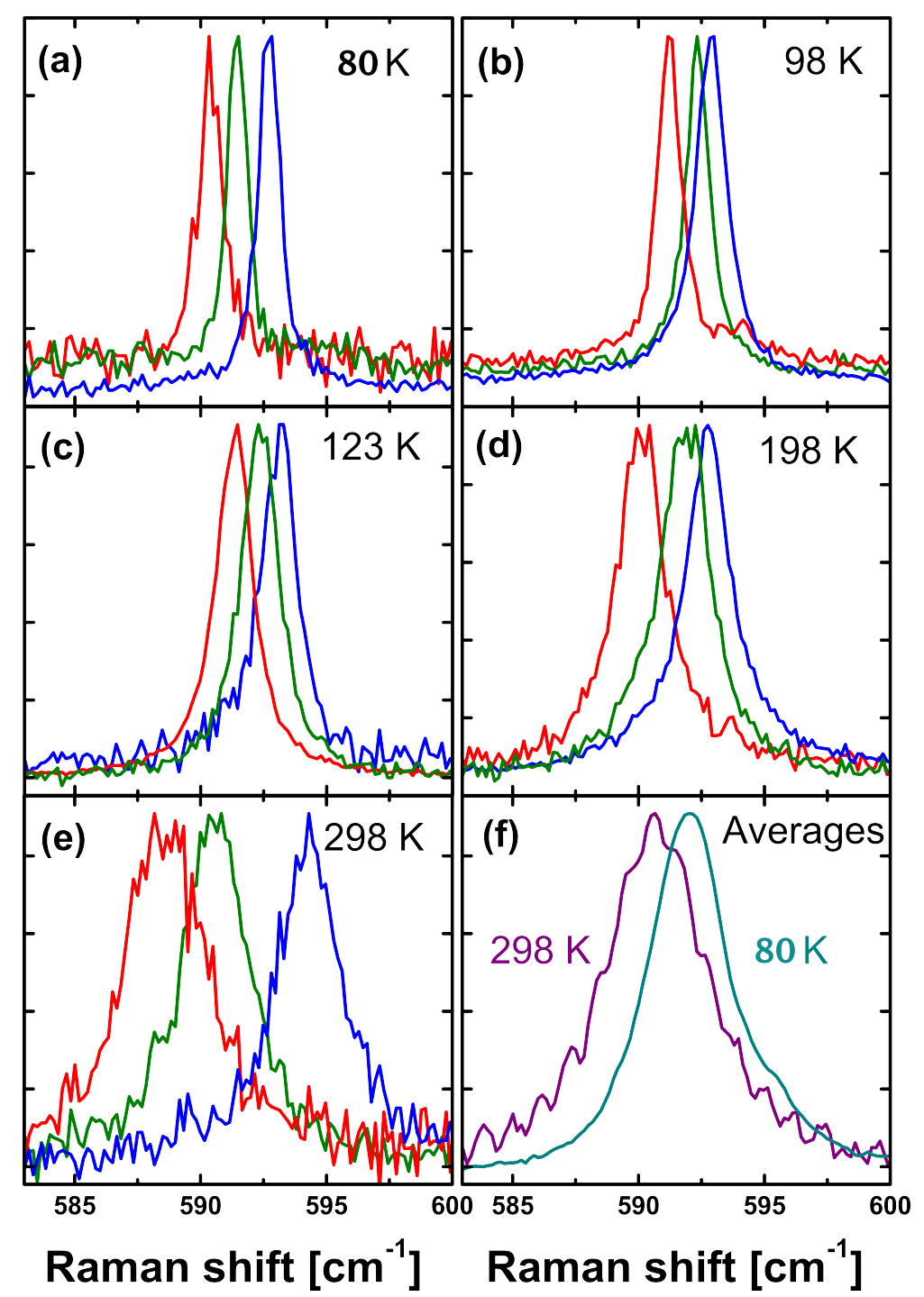

Figure 4.8: (a-e) Representative SM-SERS signals at different temperatures (for the $590 \mathrm{~cm}^{-1}$ mode of NB, $633 \mathrm{~nm}$ laser excitation, $2.5 \mathrm{~mW}$ and $1 \mathrm{~s}$ integration time; all spectra are normalized in intensity for better visualization). The change in (homogeneous) broadening of the peaks with $T$ is readily seen by eye. The frequency variations from one SMevent to another are, however, sufficiently large to wash out most of the information on the homogeneous broadening in the average spectra (for an ensemble of molecules), as shown in (f) for the two extreme temperatures. The small red-shift in peak frequency with increasing temperature is visible in both the average spectra and the average position of SMspectra. 


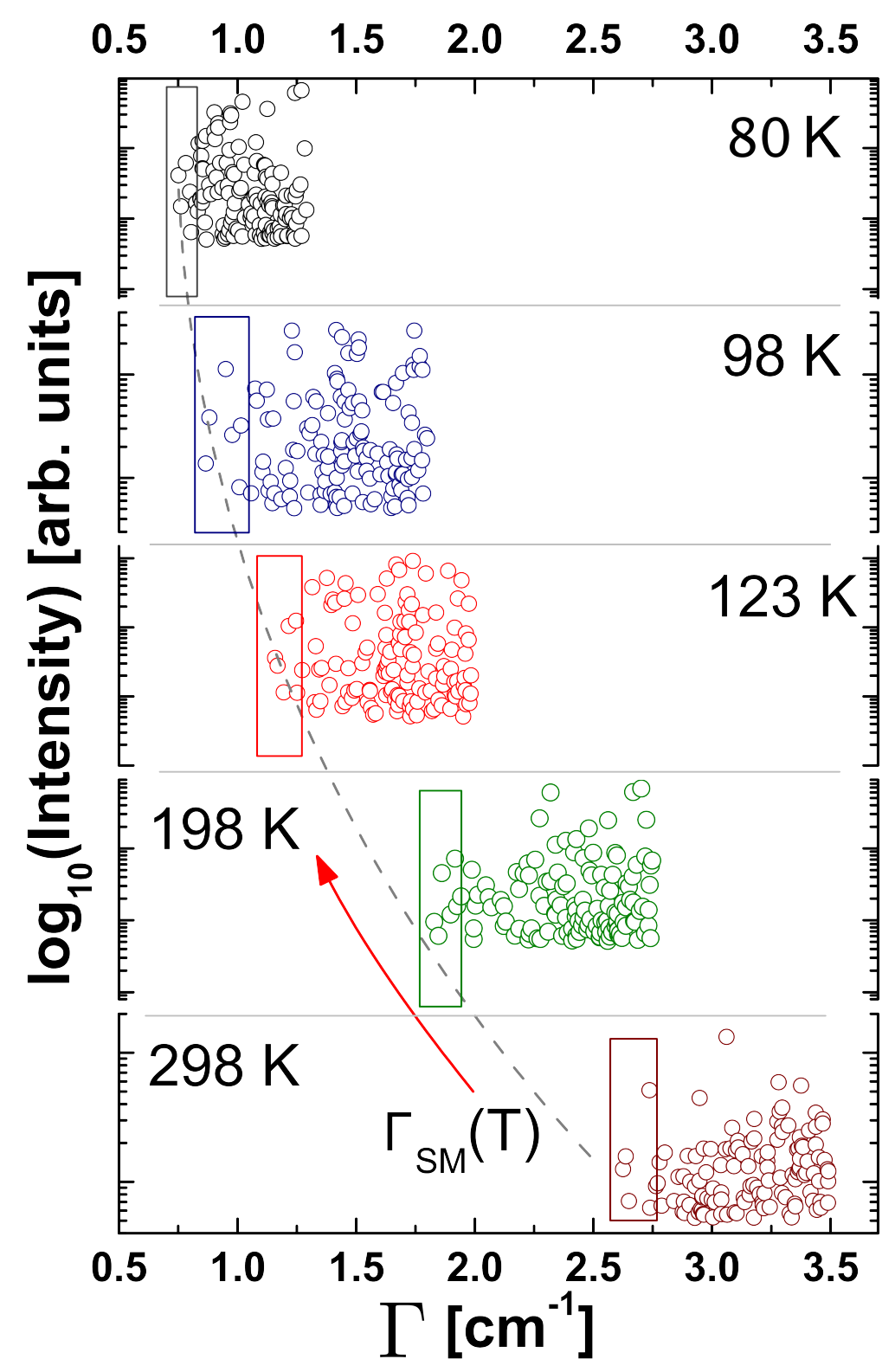

Figure 4.9: FWHM (Г) of different SERS events for the $590 \mathrm{~cm}^{-1}$ mode of NB for different temperatures. The vertical axis is logarithmic and, therefore, the different events represent signals with widely varying intensities. At all temperatures a clear minimum threshold broadening is observed (boxes). Events at this threshold broadening have a pure Lorentzian lineshape, and correspond to the minimum possible width that a SM can have at a specific temperature. The variation of this threshold $\Gamma_{\mathrm{SM}}(T)$ provides a measurement of the dependence of the homogeneous broadening as a function of temperature (schematically shown by a dashed line). 
when using dried colloids as SERS substrate. A clear threshold for the narrowest events was revealed by the analysis (there is a minimum width that an event can have) and this minimum width increases steadily as a function of $T$. Moreover, these narrowest spectra (examples of which are shown in Fig. 4.8(a-e)) are all pure Lorentzian, within the uncertainties of the fits.

This minimum broadening, $\Gamma_{\mathrm{SM}}(T)$ is therefore assigned to the single molecule (homogeneous) broadening of the $590 \mathrm{~cm}^{-1}$ Raman mode of Nile blue on the Ag surface at a given temperature. By contrast, spectra whose fit resulted in a much larger FWHM than the threshold for a given temperature, often exhibit (after direct inspection) a double or multipeak lineshape. These can therefore be attributed to multi-molecule events [51]. The remaining spectra, exhibiting a single peak of larger FWHM than the threshold are also probably two-molecule events with closely spaced Raman frequencies, but we cannot exclude the possibility that some of these are SM-spectra with a slightly larger homogeneous broadening than the others.

Having identified SM-events, it is possible to study their frequency spread at a fixed $T$ by plotting a histogram of their frequency positions, as shown in Fig. 4.10 (for the two extreme temperatures $80 \mathrm{~K}$ and $298 \mathrm{~K}$ ). From these histograms, we can conclude that the magnitude of the frequency shifts from one molecule to another has little (if any) temperature dependence and this narrows down their possible origin as surface interactions. This is in clear contrast with the decrease of $\Gamma_{\mathrm{SM}}$ from $\sim 2.7 \mathrm{~cm}^{-1}$ to as little as $\sim 0.85 \mathrm{~cm}^{-1}$ at $80 \mathrm{~K}$ as seen in Fig. 4.9). The small variation of the average broadening is well accounted for by a dominant constant contribution from frequency variations, convoluted with a smaller temperature dependent homogeneous linewidth. This reinforces the idea that only SM-measurements can directly access the homogeneously broadened spectra.

Figure 4.11 summarizes the measured temperature dependence of the homogeneous $\Gamma_{\mathrm{SM}}$ and inhomogeneous $\Gamma_{\text {ave }}$ broadening, together with the average Raman frequency $\Delta \bar{\nu}$. We first note that the small linewidths observed here place a lower limit on the dephasing $T_{2}^{\prime}$ and population 


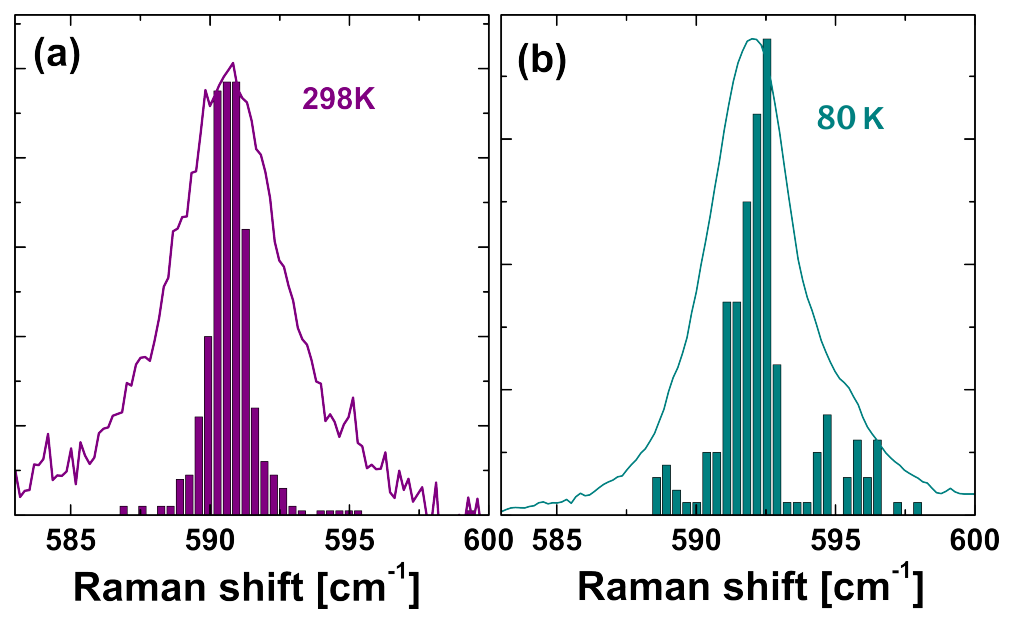

Figure 4.10: Histograms of the Raman peak positions for SM-SERS events at 80 and $298 \mathrm{~K}$. Superimposed is also the actual average spectrum for these temperatures. The frequency variations of SM-events dominates the linewidth of the average, which masks the true homogeneous broadening and its temperature dependence. Rare events at $595 \mathrm{~cm}^{-1}$ are tentatively attributed to natural isotopic substitutions or contamination by a similar (but not identical to NB) molecular species (e.g. a by-product of the dye synthesis reaction).

relaxation time $T_{1}$ of the $590 \mathrm{~cm}^{-1}$ vibration (see Section 4.2.1), from the fundamental relation:

$$
\Gamma=\frac{1}{2 \pi c}\left(\frac{1}{T_{1}}+\frac{2}{T_{2}^{\prime}}\right)
$$

where $\Gamma$ is in $\mathrm{cm}^{-1}$.

For example, at $298 \mathrm{~K} \Gamma_{\mathrm{SM}} \sim 2.7 \mathrm{~cm}^{-1}$ implies $T_{2}^{\prime} \geq 4 \mathrm{ps}$ and $T_{1} \geq 2 \mathrm{ps}$ while at $80 \mathrm{~K} \Gamma_{\mathrm{SM}} \sim 0.9 \mathrm{~cm}^{-1}$ implies $T_{2}^{\prime} \geq 12 \mathrm{ps}$ and $T_{1} \geq 6 \mathrm{ps}$.

The observed simultaneous temperature dependence of $\Gamma_{\mathrm{SM}}$ and $\Delta \bar{\nu}$ is suggestive of the vibrational energy exchange (VEE) model (see Section 4.2.4). We constructed Arrhenius plots for both the linewidth and frequency position and then extracted the activation energy $E_{B}$ of the dominant exchange mode which is likely to be responsible for the tem- 


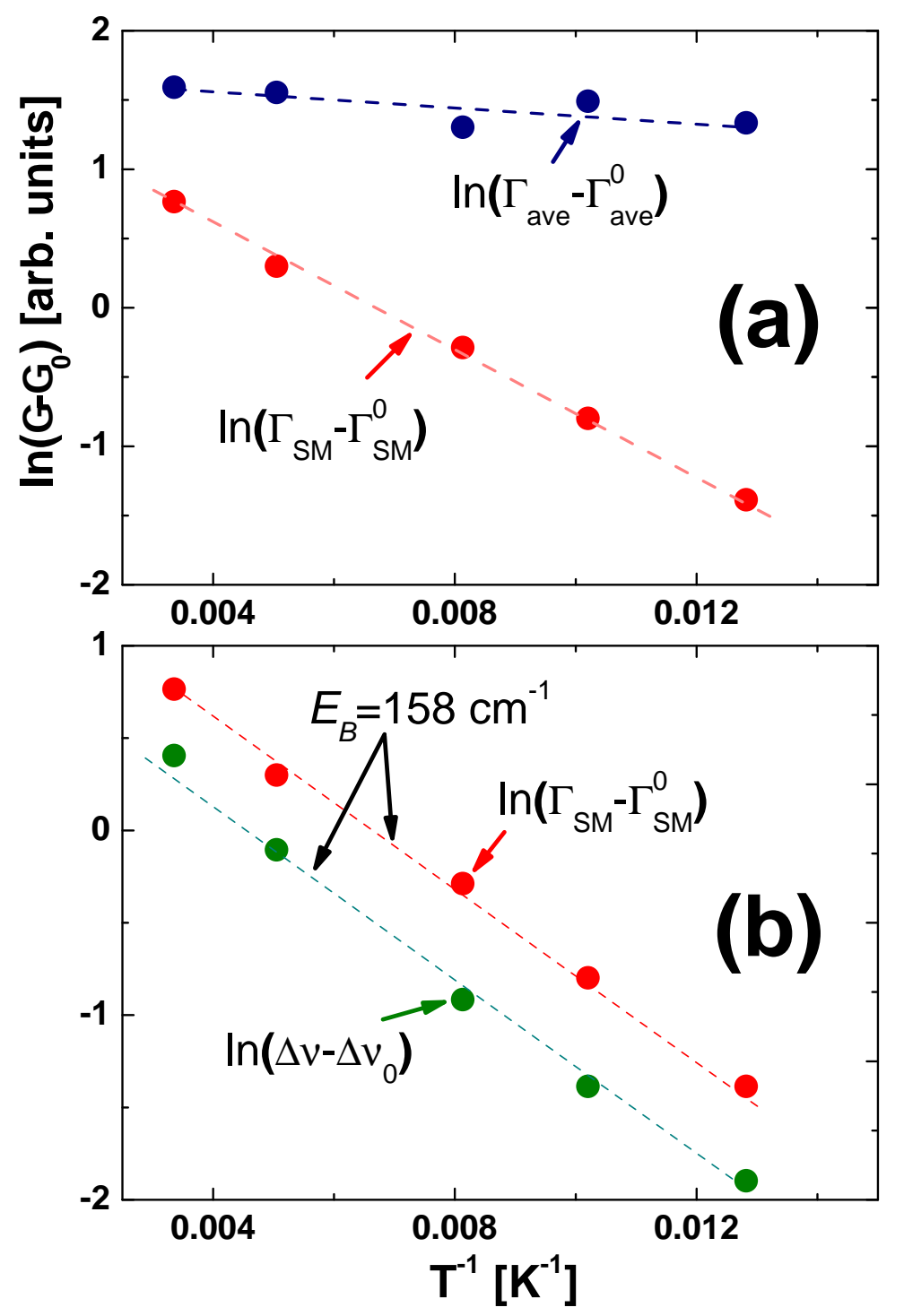

Figure 4.11: (a) Arrhenius plots for the temperature dependence of the homogeneous broadening $\Gamma_{\mathrm{SM}}(T)$ (red circles) as opposed to the linewidth of the average signal $\Gamma_{\text {ave }}(T)$ (blue circles). The temperature dependence of the average signal is weaker because it is dominated by the inhomogeneous broadening. (b) The temperature dependence of the average Raman shift $\Delta \bar{\nu}(T)$ (green circles) and that of the homogeneous broadening $\Gamma_{\mathrm{SM}}(T)$ (red circles) can be fitted using the vibrational energy exchange model (dashed lines). From these, the energy of the exchange mode $E_{B}$ is found to be $\sim 158 \mathrm{~cm}^{-1}$, indicating a dominant anharmonic coupling to the substrate $(\mathrm{Ag})$ phonons (the Debye frequency of $\mathrm{Ag}$ is $\left.\sim 160 \mathrm{~cm}^{-1}\right)$. 
perature dependence of $\Gamma_{\mathrm{SM}}$ and $\Delta \bar{\nu}$ for the $590 \mathrm{~cm}^{-1}$. Namely:

$$
\begin{aligned}
& \Gamma_{\mathrm{SM}}=\Gamma_{0}+\frac{(\delta \omega \tau)^{2}}{1+(\delta \omega \tau)^{2}} \frac{1}{\pi c \tau} \exp \left(-E_{B} /(k T)\right) \\
& \Delta \bar{\nu}=\Delta \bar{\nu}_{0}+\frac{\delta \omega \tau}{1+(\delta \omega \tau)^{2}} \frac{1}{2 \pi c \tau} \exp \left(-E_{B} /(k T)\right)
\end{aligned}
$$

where $\delta \omega$ represents the coupling strength between the Raman and the exchange modes, $\tau$ is the relaxation time of the exchange mode, and $E_{B}$ its energy. As shown in Fig. 4.11, a satisfactory fit to both quantities is obtained using the parameters: $\Gamma_{0}=0.65 \mathrm{~cm}^{-1}, \Delta \bar{\nu}_{0}=592.1 \mathrm{~cm}^{-1}$, $\tau=0.77 \mathrm{ps}, \delta \omega \tau=0.67$, and $E_{B}=158 \mathrm{~cm}^{-1}$. Uncertainties in these parameters are mainly associated with the determination of the limit of $\Gamma_{\mathrm{SM}}$ at low temperatures from Fig. 4.9. Nevertheless, it is interesting to note that $E_{B}$ closely matches the Debye frequency of silver. This suggests a natural interpretation: the dominant contribution to the temperature dependence of the homogeneous broadening is through anharmonic coupling to the phonons of the Ag substrate; by vibrational energy exchange between the $590 \mathrm{~cm}^{-1}$ mode of Nile blue and the phonons of Ag, dephasing of the $590 \mathrm{~cm}^{-1}$ mode occurs. This is indeed more likely than population relaxation through multi-phonon excitation in the silver substrate (because the Debye frequency is not an obvious multiple of the Raman one and thus a direct population decay is less likely to happen) . The dephasing time associated with this process can be estimated from $\Gamma_{\mathrm{SM}}-\Gamma_{0}$ to be $\sim 5.2 \mathrm{ps}$ at room temperature.

\subsubsection{Application to the temperature dependence of the homogeneous broadening of the $2230 \mathrm{~cm}^{-1}$ mode of rhodamine 800}

The previous section has proven that SM-SERS allows experimental investigation of the anharmonic coupling of a mode of a molecule with other modes or the underlying substrate and the relaxation channels a vibration is likely to take.

From here, we carry out a similar study on another resonant dye, 


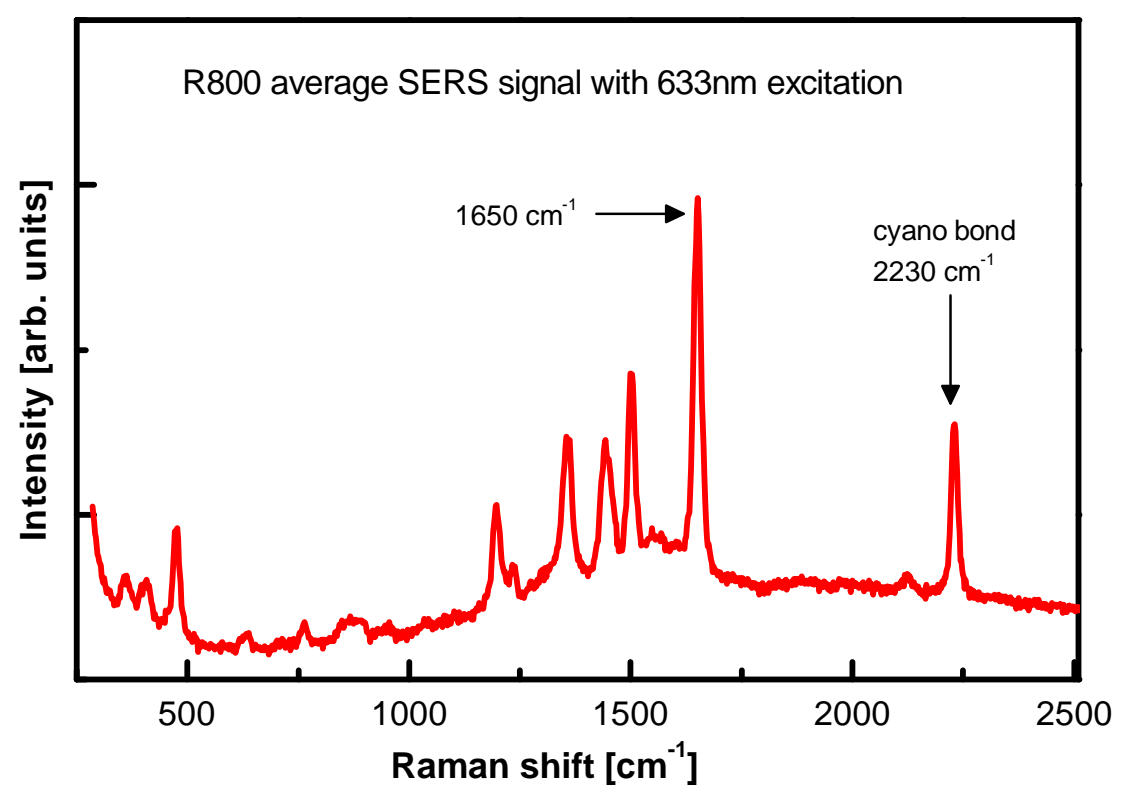

Figure 4.12: Average SERS spectrum of rhodamine 800 at $20 \mathrm{nM}$, excitation wavelength $633 \mathrm{~nm}$, objective $\times 100$, integration time $0.1 \mathrm{~s}$.

rhodamine 800 (R800), and see if we could unravel subtle interactions between this molecule and the $\mathrm{Ag}$ substrate or intramolecular interactions between modes. Usually, for medium-size to large molecules, the eigenvectors that produce Raman active modes will be extended over many atoms [1], but there are some exceptions as for the $\sim 2230 \mathrm{~cm}^{-1}$ mode of $\mathrm{R} 800$ (for a $\mathrm{R} 800$ with ${ }^{12} \mathrm{C} \equiv{ }^{14} \mathrm{~N}$ ). The carbon-nitrogen triple bond in the structure of R800 is a strong bond that produces a relatively isolated and localized stretch vibration which appears in the high energy part of the Raman spectrum. Moreover, the peak associated to this mode is narrow enough for us to expect to resolve its true homogeneous broadening within the broadening of the average (multi-molecule) peak. Rhodamine 800 was thus thought to be a good candidate to carry out a study similar to the previous one and it would be interesting to see whether the temperature dependence of the homogeneous broadening of such an isolated and energetic mode would produce a different outcome.

The aim was also to compare the temperature dependence of the broadening of the $2230 \mathrm{~cm}^{-1}$ mode to the temperature dependence of the 
linewidth of a lower energy mode of the molecule (the $1650 \mathrm{~cm}^{-1}$ peak) which is also relatively sharp and intense and see whether they were exhibiting a different behavior in their relaxation processes. Figure 4.12 shows an average SERS spectrum of rhodamine 800 at $633 \mathrm{~nm}$.

The sample preparation was the same as described in Section 2.3. We used either poly-L-lysine coated Si wafers or quartz slides onto which a solution of Lee \& Meisel silver colloids + R800 $5 \mathrm{nM}$ was drop cast then siphoned off leaving a sparse collection of Ag clusters on the surface. All the maps were recorded on the Labram spectrometer with $633 \mathrm{~nm}$ excitation wavelength, $\times 100$ LWD objective and 1800l/mm gratings. We used the latter instead of the $2400 \mathrm{l} / \mathrm{mm}$ used for the Nile Blue study because the latter one was no longer available. It was not a significant loss of resolution $\left(0.25 \mathrm{~cm}^{-1}\right.$ per pixel at $633 \mathrm{~nm}$ for the $1800 \mathrm{l} / \mathrm{mm}$ gratings instead of $0.17 \mathrm{~cm}^{-1}$ for the $2400 \mathrm{l} / \mathrm{mm}$ grating).

For each mode, $1650 \mathrm{~cm}^{-1}$ and $2230 \mathrm{~cm}^{-1}$, maps with $5 \mu \mathrm{m}$ steps and $1 \mathrm{~s}$ acquisition time were recorded at $80 \mathrm{~K}, 100 \mathrm{~K}, 150 \mathrm{~K}, 200 \mathrm{~K}, 225 \mathrm{~K}$, $250 \mathrm{~K}$ and $300 \mathrm{~K}$. As a first step, all the spectra were fitted to a pseudoVoigt lineshape and the spectra with a small signal-to-noise ratio were discarded from the statistics. We used then the FWHM $\Gamma$ obtained from the fits to discriminate SM-events.

As previously appreciated for Nile blue, for both of the modes of R800, there was a clear threshold in the minimum width the narrowest events could exhibit and this minimum $\Gamma$ was increasing for increasing temperatures. Fig. 4.13 shows the temperature dependence of the homogeneous broadening $\Gamma_{\mathrm{SM}}$ for both modes of $\mathrm{R} 800$.

However, the tentative representation of the data by Arrhenius plots was not conclusive. While a fit to the VEE model taking the exchange mode to be $158 \mathrm{~cm}^{-1}$ was satisfactory for the $1650 \mathrm{~cm}^{-1}$ mode, the fits were not trustworthy for the $2230 \mathrm{~cm}^{-1}$ mode data. Several "good enough" fits could be obtained for the $2230 \mathrm{~cm}^{-1}$ mode with very different parameters and it would be quite impossible to choose one route or the other with confidence.

Hence no satisfactory conclusion could be drawn concerning the comparative behavior of these two particular modes of rhodamine 800. Ta- 


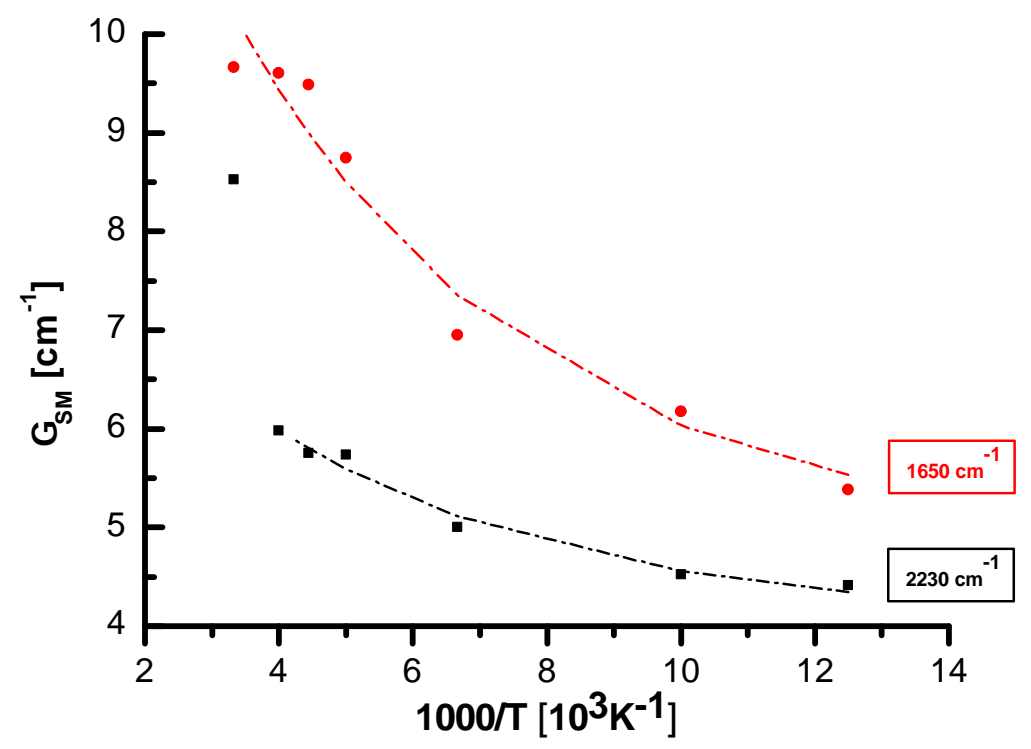

Figure 4.13: Temperature dependence of the homogeneous $\Gamma_{\mathrm{SM}}$ for the $1650 \mathrm{~cm}^{-1}$ mode ( in red) and the $2230 \mathrm{~cm}^{-1}$ mode (in black) of R800 together with exponential fits (dash dotted lines).

ble 4.1 shows the results of the fit when the room temperature data for the $2230 \mathrm{~cm}^{-1}$ mode are left out and the activation energy is constrained to be $158 \mathrm{~cm}^{-1}$.

\subsection{Conclusion}

This chapter demonstrates that SM-SERS can be a extraordinary tool to access properties of a molecule which would otherwise be hidden due to the ensemble averaging inherent to less sensitive techniques. The simplicity of the experimental set-up should be here, again, emphasized; frequency domain conventional CW Raman spectroscopy is used to get values of lifetimes of the order of the ps; a complicated ultrafast set-up would be necessary to get that resolution in the time domain. Such a study is one among many which are achievable now that SM-SERS is becoming an established technique. 


\begin{tabular}{c|cc} 
Mode & $1650 \mathrm{~cm}^{-1}$ & $2230 \mathrm{~cm}^{-1}$ \\
\hline \hline $\mathrm{E}_{B}\left(\mathrm{~cm}^{-1}\right)$ & 158 & 158 \\
\hline$\omega_{0}$ & 1652.11 & 2231.50 \\
\hline$\delta \omega \tau$ & 0.76 & 0.24 \\
\hline$\delta \omega\left(\mathrm{cm}^{-1}\right)$ & 11.86 & 10.57 \\
\hline$\tau(\mathrm{ps})$ & 1.02 & 0.35
\end{tabular}

Table 4.1: Exchange mode parameters obtained by fits to the VEE model similarly to Sec. 4.3.3. 


\section{Chapter 5}

\section{Single-molecule Surface Enhanced Raman Spectroscopy of $\mathrm{C}_{60}$}

\section{$5.1 \quad$ Introduction}

Carbon is polymorphic: it is a remarkable element showing a variety of stable forms ranging from diamond and graphite to fullerenes and 1D conducting and semi conducting carbon nanotubes. While diamond which has a three dimensional covalent crystal structure - is the hardest of all bulk materials and has the highest thermal conductivity, graphite consists of carbon layers with covalent and metallic bonding which are stacked and linked by weak van der Waals interactions and is the stable phase of carbon under ambient conditions and in bulk form.

In the mid 1980s, Smalley and co-workers at Rice University developed the chemistry of fullerenes [81] and were the first to report the production of a "remarkably stable cluster consisting of 60 carbon atoms" which was obtained by graphite vaporization under laser irradiation. A few years later, their discovery led to the synthesis by Iijima et al. of carbon nanotubes which are long, slender fullerenes and can be visualized as a sheet of graphite which has been rolled into a tube [82]. Among 
the many applications they enable: the use of carbon nanotubes as gas storage components, STM probes, field emission sources, high power electrochemical capacitors, chemical sensors, components of solar cells, transistors, etc.

The closed cage molecule $\mathrm{C}_{60}$, also called "Buckminster ball" ${ }^{1}$, and other fullerene molecules have attracted considerable interest in the past three decades because of their unique structure and exceptional material properties: optical, thermal, electronic and chemical. $\mathrm{C}_{60}$ finds today many applications that include being one of the best known electron acceptors in organic solar cells [83]. The fullerene properties (including those of carbon nanotubes) have been studied to an impressive level of detail [84]. The structure of the regular truncated icosahedron was already known to Leonardo da Vinci and German painter and mathematician Albrecht Dürer in about the year 1500 . In the $20^{\text {th }}$ century, there was a number of theoretical suggestions for icosahedral molecules [85, 86] and it was proposed in 1970 that an icosahedral $\mathrm{C}_{60}$ might be stable chemically [87] but it was not until the experimental work of Kroto and Smalley in the mid 1980s that the stability of $\mathrm{C}_{60}$ molecule in gas phase was established. At that time, mass spectroscopy was used as the main characterization tool for fullerenes and several experiments, like chemical stability or photofragmentation studies, were carried out to support the cage-like structure of $\mathrm{C}_{60}$. But the direct evidence of the icosahedral symmetry came from nuclear magnetic resonance experiments [88] where a single line was observed consistent only with one type of chemical site for carbon atoms in this molecule.

In studying the vibrational spectra of $\mathrm{C}_{60}$, the major experimental techniques are: inelastic neutron scattering, electron energy loss spectroscopy (EELS), infrared and Raman spectroscopy. The Raman spectrum of $\mathrm{C}_{60}$ (in its various forms) in particular has already been investi-

\footnotetext{
${ }^{1}$ In homage to Richard Buckminster Fuller (1895 - 1983) an architectural modeler who popularized the geodesic domes. As the discovery of the fullerene family came after the buckminsterfullerene, the short name "fullerene" is used to name it, with the suffix "-ene" indicating that each C atom is covalently bonded to three others.
} 


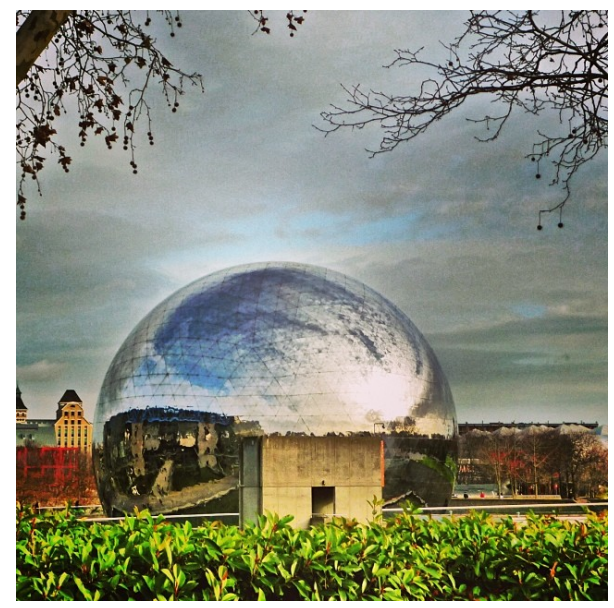

(a) La Geode

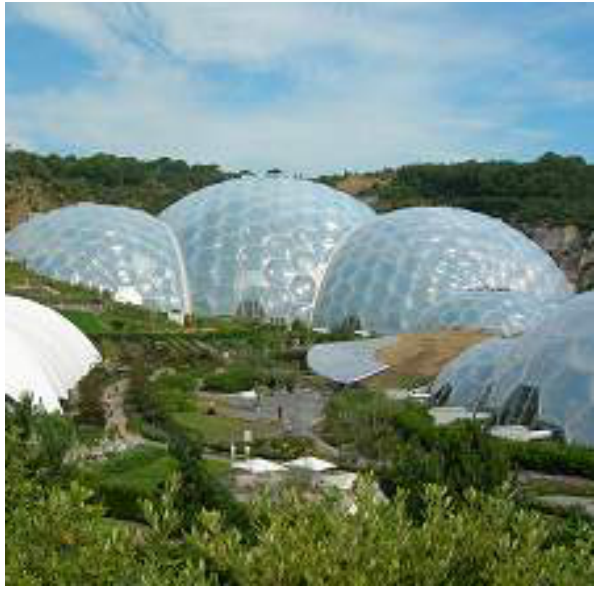

(b) Eden Project

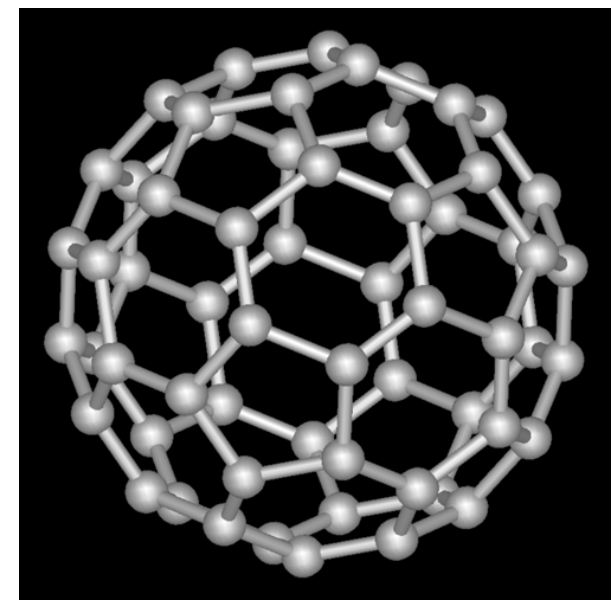

(c) $\mathrm{C}_{60}$ molecule

Figure 5.1: Structural analogy between the Buckminster ball molecule (c) and the geodesic domes (a) "La Geode" at La Villette near Paris (image obtained from https://www.flickr.com/photos/patrick nouhailler/11653321646 under the license creativecommons.org/ licenses/by-sa/2.0) and (b) "The Eden Project" at St Austell in Cornwall (obtained from http://commons.wikimedia.org/wiki/File: Eden_project.JPG).

gated in detail $[89,90,91,92,93,94,95,96,97,98]$ and while the original emphasis of the earlier Raman studies was on the confirmation of group theory predictions for the proposed icosahedral symmetry of the molecule [96, 95], later Raman studies addressed other issues such as photopolymerization [99], crystal dynamics [100], optical transitions [101, 102] and 
isotope effects $[97,103]$ : it turns out that the highly symmetric character of the $\mathrm{C}_{60}$ molecule has dramatic consequences for its vibrational isotope effects and this will be a key point for its single molecule SERS detection as we shall see in this chapter.

\subsection{Structure of $\mathrm{C}_{60}$, icosahedral point group $\mathbf{I}_{h}$ and Raman active modes}

\subsubsection{Structure of the $\mathrm{C}_{60}$ molecule}

The 60 carbon atoms of the $\mathrm{C}_{60}$ molecule are located at the vertices of a truncated icosahedron where all carbon sites are equivalent. An icosahedron is a regular polyhedron with 20 identical equilateral faces, 30 edges and 12 vertices. To obtain a regular truncated icosahedron, one has to cut the 12 vertices such that one third of each edge is cut off at both ends (see Figure 5.2 (a) and (b)). The regular truncated icosahedron has 60 equivalent vertices, 90 edges of the same length, 12 regular pentagonal faces and 20 regular hexagonal faces to form a closed shell which is consistent with Euler's polyhedron formula:

$$
f+v=e+2
$$

where $f, v$ and $e$ are respectively the number of faces, vertices and edges of the convex polyhedron. Furthermore, from Equation 5.1 a simple rule for closed cage molecules follows: a closed cage containing only hexagonal and pentagonal faces requires the number of pentagonal faces to be exactly 12 and the number of hexagonal faces can be arbitrary. Inspection of Figure 5.2 (c) shows that every pentagon is surrounded by five hexagons. Two single electron-poor $\mathrm{C}-\mathrm{C}$ bonds are located along a pentagonal edge between pentagon and hexagon whose length is denoted $\mathrm{a}_{5}$ and has been measured by NMR [104] to be $1.46 \AA$ (and $1.455 \AA$ by neutron scattering [105]). The third bond is an electron-rich double bond located at the fusion between two hexagons and has a bond length denoted $\mathrm{a}_{6}$ which has been measured to be $1.40 \AA$ by NMR and $1.39 \AA$ by 
neutron diffraction. The vertices of the $\mathrm{C}_{60}$ molecule therefore do not form strictly speaking a regular truncated icosahedron; however, in most of the descriptions of $\mathrm{C}_{60}$, the small discrepancy between $\mathrm{a}_{5}$ and $\mathrm{a}_{6}$ can be neglected [84]. Moreover, the icosahedral symmetry $I_{h}$ is preserved in spite of unequal values for $\mathrm{a}_{5}$ and $\mathrm{a}_{6}$.

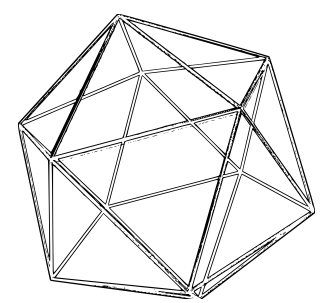

(a)

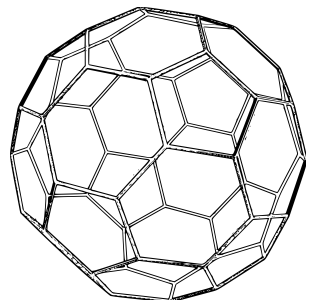

(b)

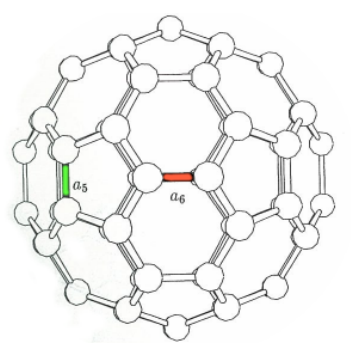

(c)

Figure 5.2: (a) Regular icosahedron,(b) regular truncated icosahedron and (c) $\mathrm{C}_{60}$ molecule showing single bonds $\left(\mathrm{a}_{5}\right)$ and double bonds $\left(\mathrm{a}_{6}\right)$.

$\mathrm{C}_{60}$ has a mean ball diameter of $7.10 \AA$ and an outer diameter of $10.34 \AA$, assuming that it has a thickness of $3.35 \AA$, which comes from the interplanar distance between two graphite layers [106]. While optimizing the geometry of the $\mathrm{C}_{60}$ molecule by Density Functional Theory (DFT), using the computational chemistry software Gaussian0 $9^{\circledR}$, we found the optimized bond lengths to be $\mathrm{a}_{5}=1.452 \AA$ and $\mathrm{a}_{6}=1.392 \AA$ (see Fig.5.3 and Section 5.4.2 for details on the DFT calculations on $\mathrm{C}_{60}$ ).

\subsubsection{Symmetry and normal modes of the $\mathrm{C}_{60}$ molecule}

Many of the exceptional properties of the Buckminster ball arise directly from its symmetry: $\mathrm{C}_{60}$ has the highest degree of symmetry of all molecules. The truncated icosahedron structure belongs to the icosahedral symmetry point group $\mathrm{I}_{h}$.

In calculating the normal modes and frequencies of vibrations, symmetry considerations can reduce enormously the labor in the calculations. Furthermore, without any other information whatsoever, the symmetries and geometry of a molecule can be used to determine the number of nor- 


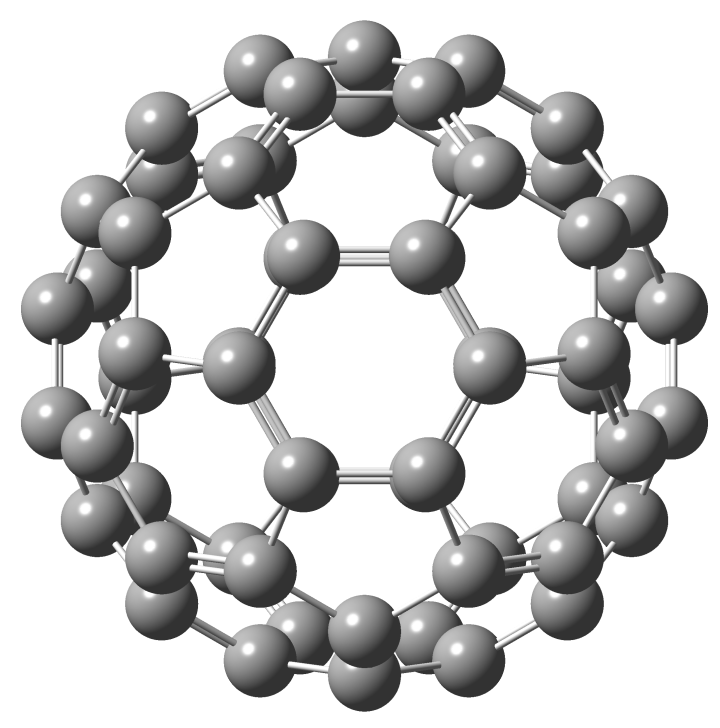

Figure 5.3: Optimized structure of $\mathrm{C}_{60}$ calculated by DFT.

mal frequencies, their degeneracies, whether they are infrared or Raman active (by considering the respective selection rules of these processes) and the polarization properties of the Raman modes. The determination of these different properties arises directly from group theory considerations.

\section{Symmetry considerations of the $\mathrm{C}_{60}$ molecule}

The molecule $\mathrm{C}_{60}$ belongs to the point group $\mathrm{I}_{h}$ [84] which contains 120 symmetry operations arranged into 10 conjugacy classes. These classes are the identity operator, the 24 fivefold rotations $\left(\mathrm{C}_{5}\right.$ and $\left.\mathrm{C}_{5}^{2}\right)$ going through the centers of the 12 pentagonal faces, the 20 threefold rotations $\mathrm{C}_{3}$ going through the centers of the 20 hexagonal faces, the 15 twofold $\mathrm{C}_{2}$ rotations going through the centers of the 30 hexagon-hexagon edges, the inversion operator and all the classes formed by the product of these five-, three-, twofold rotations with the inversion operator. $\mathrm{C}_{60}$ has 174 vibrational degrees of freedom (174 normal eigenvectors). Since icosahedral symmetry gives rise to a large number of degenerate modes, only 46 
modes have distinct frequencies. The point group $\mathrm{I}_{h}$ has 10 irreducible representations as given in Table 5.1. These results come from group theoretical arguments. Only the 8 fivefold degenerate gerade modes of symmetry $H_{g}$ and the 2 non-degenerate gerade modes of symmetry $A_{g}$, so 10 mode frequencies are Raman active in the first order. The subscripts $g$ or $u$ stand for gerade (even) and ungerade (odd), referring to the symmetry of the eigenvector under the action of the inversion operator. The $A_{g}(1)$ 'breathing' mode at $492 \mathrm{~cm}^{-1}$ involves identical radial displacements for all the 60 carbon atoms whereas the $A_{g}(2)$ 'pentagonal pinch' mode at $1469 \mathrm{~cm}^{-1}$ involves tangential displacements with contraction of the pentagonal rings and expansion of the hexagonal rings. The 8 fivefold degenerate $H_{g}$ modes are harder to visualize on a figure, their frequencies range from 273 to $1578 \mathrm{~cm}^{-1}$.

\begin{tabular}{c|c|cccccccccc} 
& $N_{\omega}{ }^{a}$ & $A_{g}{ }^{b}$ & $F_{1 g}$ & $F_{2 g}$ & $G_{g}$ & $H_{g}{ }^{c}$ & $A_{u}$ & $F_{1 u}$ & $F_{2 u}$ & $G_{u}$ & $H_{u}$ \\
\hline \hline $\mathrm{C}_{60}$ & 46 & 2 & 3 & 4 & 6 & 8 & 1 & 4 & 5 & 6 & 7
\end{tabular}

Table 5.1: Table of representations: symmetry types of vibrational modes of $\mathrm{C}_{60}$, adapted from [84]

\footnotetext{
${ }^{a}$ Number of modes with distinct frequencies

${ }^{b}$ Raman active mode only seen in $(\|\|$,$) polarization$

${ }^{c}$ Raman active mode seen in both polarization configurations
}

Table 5.2 gives the frequencies of the 10 Raman-active modes of the isolated $\mathrm{C}_{60}$ molecule [107].

Although the vibrational frequencies given in Table 5.2 are all for an isolated molecule, they are usually compared to experimental data on $\mathrm{C}_{60}$ molecular vibrations in the solid state [89]. The justification for this is that $\mathrm{C}_{60}$ in solid state (for example the powder which can be purchased) consists in face-centered cubic crystals where the bonds between $\mathrm{C}_{60}$ molecules are van der Waals and this gives rise to very small, generally unresolved splitting of the 10 Raman lines. Hence the $2 A_{g}$ modes and the $8 H_{g}$ modes dominate the Raman spectrum of the solid state sample as can be seen in Fig. 5.4. 


\begin{tabular}{cc} 
Mode & Frequency $^{a}\left(\mathrm{~cm}^{-1}\right)$ \\
\hline$H_{g}(1)$ & 272 \\
$H_{g}(2)$ & 433 \\
$A_{g}(1)$ & 496 \\
$H_{g}(3)$ & 709 \\
$H_{g}(4)$ & 772 \\
$H_{g}(5)$ & 1099 \\
$H_{g}(6)$ & 1252 \\
$H_{g}(7)$ & 1426 \\
$A_{g}(2)$ & 1469 \\
$H_{g}(8)$ & 1575
\end{tabular}

Table 5.2: First order Raman active modes of the isolated $\mathrm{C}_{60}$ molecule ${ }^{a}$ as given in $[107]$

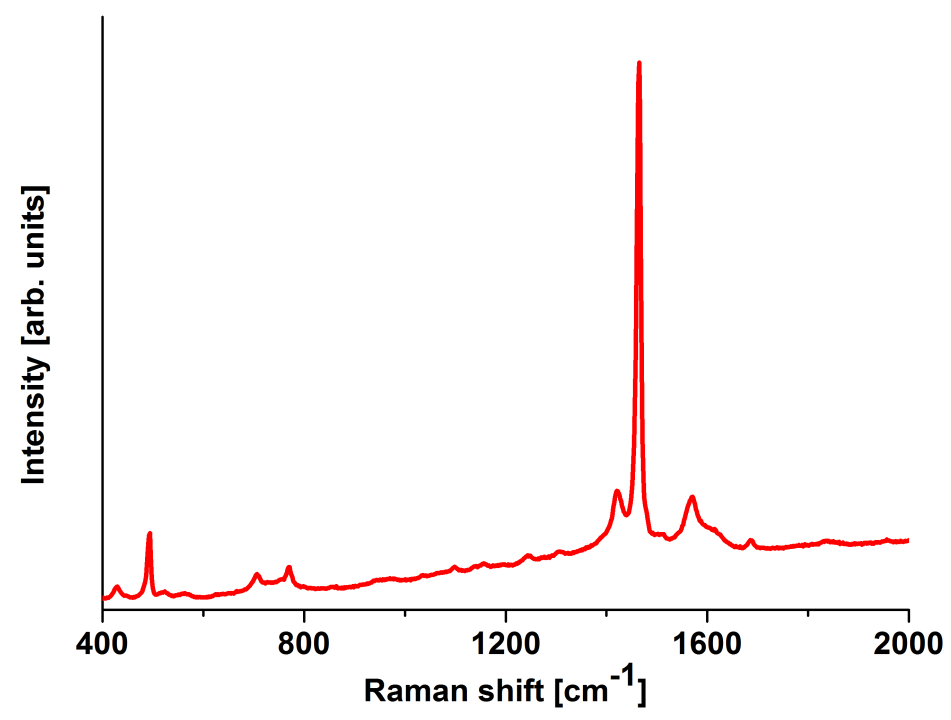

Figure 5.4: First order Raman spectrum of natural $\mathrm{C}_{60}$ powder, recorded at $514 \mathrm{~nm}$ excitation and 10 min integration. 


\subsection{Direct isotopic effect on the $A_{g}(2)$ mode frequency}

Raman spectroscopy is a very sensitive tool for probing structural changes around a symmetric molecule. In the case of $\mathrm{C}_{60}$, one obvious question that comes to mind is: what happens to the vibrational and rotational spectra when natural isotopic substitution ${ }^{13} \mathrm{C} \longrightarrow{ }^{12} \mathrm{C}$ occurs?

Carbon has two stable isotopes: ${ }^{12} \mathrm{C}$, which is $98.892 \%$ abundant, has an atomic mass of $12 \mathrm{u}$ and nuclear spin of 0 and ${ }^{13} \mathrm{C}$ which is $1.108 \%$ abundant with atomic mass $13.03 \mathrm{u}$ and a nuclear spin of $\frac{1}{2}$. The probability $p_{m}$ for $m$ isotopic substitutions to occur on a $\mathrm{C}_{60}$ molecule is given by:

$$
p_{m}=\frac{60 !}{(60-m) ! m !} x^{m}(1-x)^{60-m}
$$

where $x$ is the fractional abundance of of the isotope. Equation 5.2 stems from the probability, for each of the 60 carbon atoms, of picking ${ }^{13} \mathrm{C}$ instead of a ${ }^{12} \mathrm{C}$, the probability of which is weighted by their respective fractional abundance. Figure 5.5 shows the $m$ dependence of $p_{m}\left(\mathrm{C}_{60}\right)$ for values concentrations of $x$ equal to $1.1 \%, 20 \%$ and $30 \%$. We can see that, naturally, about $51 \%$ of $\mathrm{C}_{60}$ molecules are ${ }^{12} \mathrm{C}_{60}$ and about $48 \%$ are ${ }^{13} \mathrm{C}_{\mathrm{m}}{ }^{12} \mathrm{C}_{60-\mathrm{m}}$ with $m \geq 1$. We also see that, as $x$ increases, the distribution peak shifts towards larger $m$ - higher content in ${ }^{13} \mathrm{C}$ on average per molecule - and the distribution broadens.

These results have important consequences for both symmetry considerations and rotational levels of $\mathrm{C}_{60}$ molecules [108]. Whereas the ${ }^{12} \mathrm{C}_{60}$ molecules exhibit the highest degree of symmetry and the ${ }^{13} \mathrm{C}_{60}$ the next highest degree of symmetry, molecules as ${ }^{12} \mathrm{C}_{59}{ }^{13} \mathrm{C}_{1},{ }^{12} \mathrm{C}_{58}{ }^{13} \mathrm{C}_{2}$ have a much lower symmetry than that of the the full $\mathrm{I}_{h}$ point group; in fact, with two or more substitutions, $\mathrm{C}_{60}$ loses its entire symmetry and so, theoretically, none of the 174 vibrational modes is degenerate and in principle all of them are Raman-active. Experimentally, upon addition of ${ }^{13} \mathrm{C}$ isotopes, the rotational and rotational-vibrational spectra are not affected significantly but at ultra low temperatures $(\leq 1 \mathrm{~K})$ and the intensities of the Raman lines do not change much compared to the natural 
$\mathrm{C}_{60}$ case: the optically active modes still show strong intensities and the previously optically inactive modes remain undetectable.

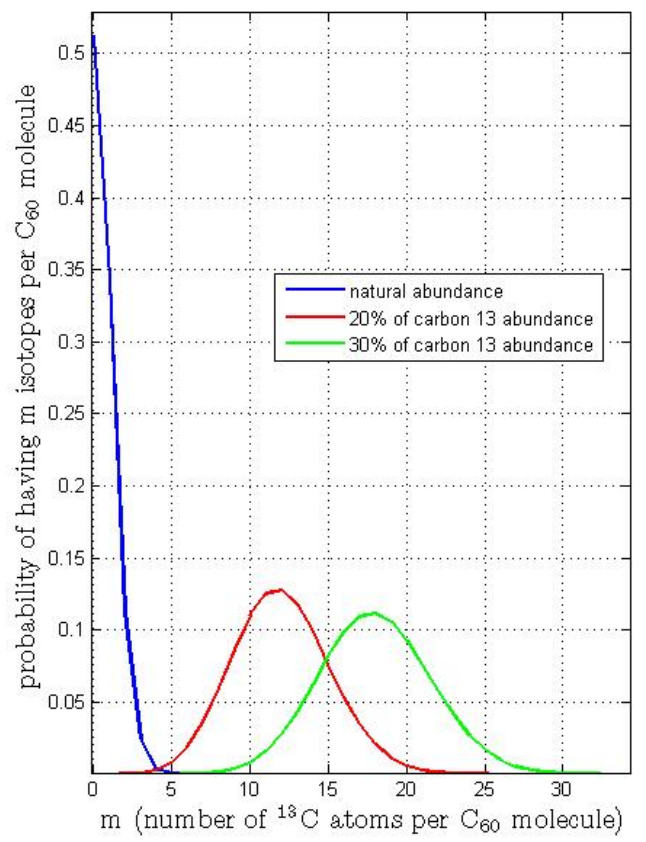

Figure 5.5: Probability of ${ }^{13} \mathrm{C} \longrightarrow{ }^{12} \mathrm{C}$ substitution for a $\mathrm{C}_{60}$ molecule.

One interesting isotope effect is the softening of the $A_{g}(2)$ mode, due to the effect a change in the mass of one or more carbon atoms has on the $\left(\frac{K}{M}\right)^{1 / 2}$ factor (which the mode frequencies depend on). This effect has been reported in 1994 by Guha et al. and is the basis of our work on single molecule SERS detection of $\mathrm{C}_{60}$. In their paper, they show that the Raman spectrum of $\mathrm{C}_{60}$ in $\mathrm{CS}_{2}$ at $30 \mathrm{~K}$ around the $A_{g}(2)$ mode $\left(1469 \mathrm{~cm}^{-1}\right)$ has a fine structure which is isomorphic to the mass spectrum of the molecule [97, 109]. They attributed the three resolved peaks to Raman scattering coming from molecules containing 0,1 or 2 ${ }^{13} \mathrm{C}$ atoms and showed that the frequency of the $A_{g}(2)$ mode undergoes a first-order shift $\Delta \omega$ induced by $n$ isotopic substitutions within nondegenerate perturbation theory:

$$
\left(\frac{\Delta \omega^{2}}{\omega_{0}^{2}}\right)_{1}=-\frac{n}{720}
$$


where $\omega_{0}$ is the frequency of the unperturbed $A_{g}(2)$ mode (for a ${ }^{12} \mathrm{C}_{60}$ ). This surprisingly simple result arises directly from the symmetry of the $A_{g}(2)$ mode and the fact that every site in the unperturbed $\mathrm{C}_{60}$ molecule is symmetry-equivalent.

Specifically, upon ${ }^{13} \mathrm{C} \longrightarrow{ }^{12} \mathrm{C}$ substitution, the force-constant matrix and the Raman polarizabilities are unchanged as the molecular electronic states are not affected by isotopic substitution- and so, determining the effects on the vibrational spectrum of any isotopic substitution by DFT once the electronic structure of the molecule has been calculated, is not costly. It is natural to try perturbation theory (non-degenerate) given how 'small' the isotopic perturbations are: the perturbation introduced by each ${ }^{13} \mathrm{C}$ is only of order $1 / 720$. The perturbed frequency for a nondegenerate mode $f$ is given by the series $[109,110]$ :

$$
\frac{\Delta \omega_{f}^{2}}{\omega_{0 f}^{2}}=\sum_{n=1,2 \ldots}\left(\frac{\Delta \omega_{f}^{2}}{\omega_{0 f}^{2}}\right)_{n}
$$

The first order correction is:

$$
\left(\frac{\Delta \omega_{f}^{2}}{\omega_{f 0}^{2}}\right)_{1}=-\chi_{0}^{t}(f) \Delta \mathbf{M} \boldsymbol{\chi}_{0}(f)
$$

where $\Delta \mathbf{M}$ is such as $\mathbf{M}_{0}+\Delta \mathbf{M}$ is the mass matrix of the perturbed molecule, $\boldsymbol{\chi}_{0}(f)$ the unperturbed eigenvector of mode $f$ and $\boldsymbol{\chi}_{0}^{t}(f)$ its transpose. Because every site in the unperturbed icosahedral $\mathrm{C}_{60}$ molecule is symmetry equivalent, the magnitude of the atomic displacements for the unperturbed totally symmetric $A_{g}$ modes are site-independent and thus for $n$ isotopic substitution we get the simple expression of the firstorder correction in Eq. 5.3. The independence of the exact positions of the ${ }^{13} \mathrm{C}$ substitutions on the overall frequency shift can be demonstrated with a direct calculation of the vibrational dynamics of $\mathrm{C}_{60}$ as shown with DFT calculation on $\mathrm{C}_{60}$ isotopologues, see Section 5.5.2. 


\subsection{Preliminary experiments}

We purchased the non-enriched $\mathrm{C}_{60}$ from Sigma-Aldrich with a purity of $99.9 \%$ and the ${ }^{13} \mathrm{C}$-enriched $\mathrm{C}_{60}$ sample from Materials \& Electrochemical Research (MER) Corporation with a purity $>98 \%$. Both samples are brown-black micro-crystalline powder, the solubility in water is $1.3 \times 10^{-11} \mathrm{mg} / \mathrm{mL}$ [111]- so is almost negligible- and the solubilities of $\mathrm{C}_{60}$ in various organic solvents at $298 \mathrm{~K}$ are given in Table 5.3:

\begin{tabular}{lc} 
Solvent & $\mathrm{C}_{60}$ solubility, mg/mL \\
\hline methanol & 0.000 \\
ethanol & 0.001 \\
dichloromethane $\left(\mathrm{CH}_{2} \mathrm{Cl}_{2}\right)$ & 0.26 \\
toluene & 2.8 \\
carbon disulfide $\left(\mathrm{CS}_{2}\right)$ & 7.9
\end{tabular}

Table 5.3: Solubility of $\mathrm{C}_{60}$ in various solvents (adapted from [112])

Before conducting SERS and SM-SERS experiments on $\mathrm{C}_{60}$, we needed to carry out some preliminary work which would help us characterize some relevant properties of $\mathrm{C}_{60}$ and get to know better the system we wanted to study (sample preparation, measurement's resolution, etc.):

* Determination of the differential Raman cross section of the $A_{g}(2)$ mode at $633 \mathrm{~nm}$ excitation written in this section $\left.\frac{d \sigma}{d \Omega}\right)_{633 \mathrm{~nm}}^{\left(A_{g}(2)\right)}:$ this knowledge is necessary to determine the enhancement factors in SERS spectra.

* Density Functional Theory (DFT) calculation of the vibrational spectrum of an isolated $\mathrm{C}_{60}$ molecule: this is interesting to calculate to, on the one hand, validate the experimental determination of $\left.\frac{d \sigma}{d \Omega}\right)_{633 \mathrm{~nm}}^{\left(A_{g}(2)\right)}$ and, on the other hand use the calculated force constant matrix to simulate the effect of isotopic substitution on the vibrational spectrum of $\mathrm{C}_{60}$. 


\subsubsection{Experimental determination of the differen- tial Raman cross-section of the $\operatorname{Ag}(2)$ mode at $633 \mathrm{~nm}$}

A fundamental question in SM-SERS is always that of the magnitude of the enhancement factor (EF) $[29,41]$. In order to be able to quantify the SM-SERS EF, it is necessary to: ensure that the observed signals are indeed from single molecules, be able to compare the integrated peak intensity of a vibration with a signal of known Raman differential cross section $(d \sigma / d \Omega)$ under the same experimental conditions, and finally know the intrinsic $d \sigma / d \Omega$ of the molecule for that specific vibration. Unfortunately, intrinsic values of $d \sigma / d \Omega$ for a specific laser excitation are very difficult to find (even for an iconic molecule like $\mathrm{C}_{60}$ ). Therefore, we concentrate first on this task.

Beside its interesting effect with regard to isotopic substitution, the $A_{g}(2)$ mode at $1469 \mathrm{~cm}^{-1}$ is the most distinguishable Raman-active fingerprint from $\mathrm{C}_{60}$. The differential cross section of this mode can be obtained in solution by a direct comparison of the Raman intensity with that of a solvent that has a nearby Raman peak with a known $d \sigma / d \Omega$ [19]. This indeed ensures that both molecules experience exactly the same experimental conditions. Inspection of Table 5.3 shows that toluene is a good solvent for $\mathrm{C}_{60}$ and the absolute differential Raman cross section of the $1002 \mathrm{~cm}^{-1}$ mode of toluene has been measured accurately to be $2.9 \times 10^{-30} \mathrm{~cm}^{2} / \mathrm{sr}$ at $633 \mathrm{~nm}[5]$. Some preliminary tests showed that if $\mathrm{C}_{60}$ is in a $1 \mathrm{mM}$ solution in toluene, $20 \mathrm{~s}$ acquisition with $\times 100 \mathrm{im}$ mersion objective at $\lambda_{\mathrm{ex}}=633 \mathrm{~nm}$ are necessary to resolve the $A_{g}(2)$ peak whose height is only $2.5 \%$ of the height of the background. But as toluene is a very volatile solvent, it evaporates over the length of the measurement (400 s as we take the average of 20 spectra of $20 \mathrm{~s}$ ) and as a consequence of the unstable $\mathrm{C}_{60}$ concentration, the integrated intensity's ratio between $\mathrm{C}_{60}$ and toluene is not constant. To avoid evaporation

problems, we decided to carry out the measurements on $\mathrm{C}_{60}$ in toluene in a quartz cell (see fig 5.6) and used the $\times 100$ LWD objective (N.A. 0.6) and focused the laser inside the cell. To determine the $d \sigma / d \Omega$ of the 
$1469 \mathrm{~cm}^{-1}$ mode of $\mathrm{C}_{60}$, we used a solution of $\mathrm{C}_{60} 1 \mathrm{mM}$ in toluene, the spectrometer was equipped with the $1800 \mathrm{l} / \mathrm{mm}$ gratings, excitation was $633 \mathrm{~nm}, P_{\text {inc }}=6.3 \mathrm{~mW}$. The Raman spectrum of the $1002 \mathrm{~cm}^{-1}$ mode of toluene was recorded with $1 \mathrm{~s}$ acquisition and 20 Raman spectra around the $1469 \mathrm{~cm}^{-1}$ peak of $\mathrm{C}_{60}$ were accumulated with $20 \mathrm{~s}$ acquisition and their average was used for determining the integrated intensity of the peak (see Fig. 5.7).

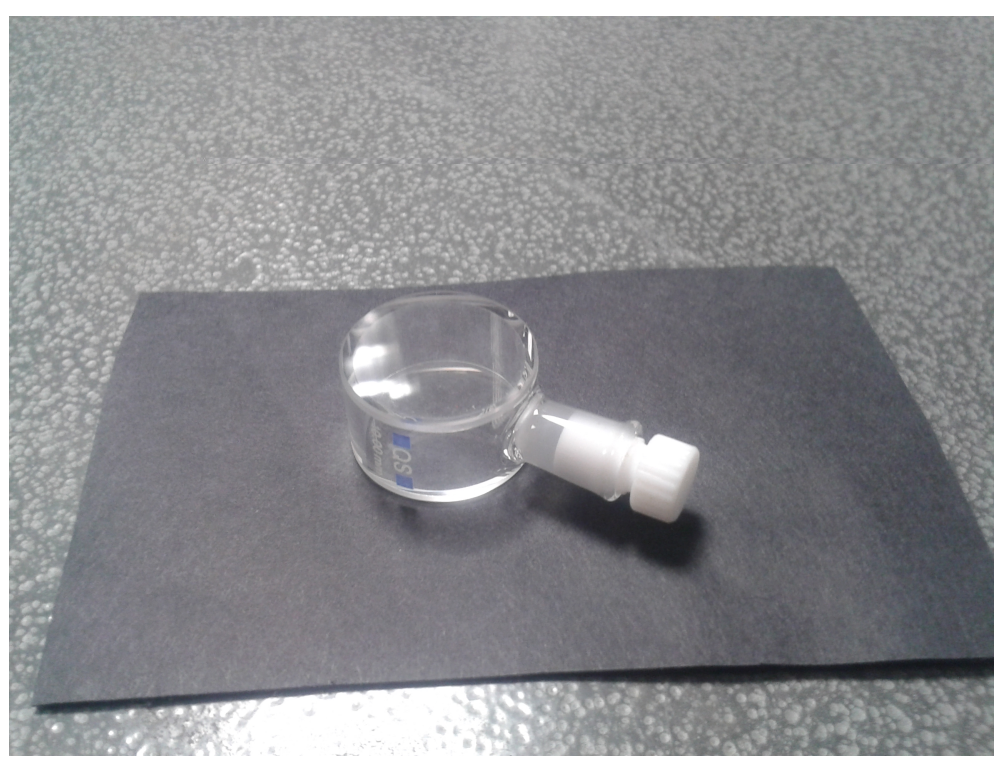

Figure 5.6: Quartz cell used for the determination of the differential Raman cross section of the $A_{g}(2)$ mode of $\mathrm{C}_{60}$ at $633 \mathrm{~nm}$.

The comparison is made between the known differential cross section of the $1002 \mathrm{~cm}^{-1}$ mode of toluene and the integrated intensity of the $1469 \mathrm{~cm}^{-1}$ pinch mode of $\mathrm{C}_{60}$ (properly scaled by the number of molecules participating in the signal and the integration time). The density of toluene is $0.86 \mathrm{~g} / \mathrm{mL}$ and its molar mass is $92.14 \mathrm{~g} / \mathrm{mol}$, hence the concentration of toluene $c_{\text {tol }}$ is:

$$
c_{\mathrm{tol}}=\frac{0.86}{92.14}=9.3 \times 10^{-3} \mathrm{M}
$$

Hence the ratio of $\mathrm{C}_{60}$ molecules versus toluene molecules is:

$$
\frac{c_{\mathrm{C}}}{c_{\mathrm{tol}}}=1.12 \times 10^{-4}
$$


Thus, knowing the latter ratio, and knowing the ratio of the integrated Raman intensities per second, we get the value for the differential Raman cross section of the pentagonal pinch mode of $\mathrm{C}_{60}$ at $633 \mathrm{~nm}$ :

$$
\left.\frac{d \sigma}{d \Omega}\right)_{633 \mathrm{~nm}}^{\left(A_{g}(2)\right)}=2.9 \times 10^{-28} \mathrm{~cm}^{2} / \mathrm{sr}
$$

The value given in Eq.5.8 is the thus the absolute differential Raman cross-section of the $A_{g}(2)$ mode of the $\mathrm{C}_{60}$ molecule in toluene at $633 \mathrm{~nm}$; if one wants to get an approximate value for the cross section of the free molecule (without the solvent), a local field correction $\left(L_{\mathrm{M}}\right)$ for the index of refraction of toluene needs to be included [19]. The local field felt by a molecule in a solvent is modified compared to the macroscopic field created by the laser excitation. For Raman scattering, it leads to a local field correction of the cross section:

$$
L_{\mathrm{M}}=\left[\frac{n_{\mathrm{M}}^{2}+2}{3}\right]^{1 / 4}
$$

where $n_{\mathrm{M}}$ is the refractive index of the medium surrounding the molecule (solvent). In the case of toluene, the refractive index is $n_{\text {tol }}=1.497$ and so $L_{\mathrm{M}} \simeq 4$, which means that the cross section of $\mathrm{C}_{60}$ in toluene is approximately four times larger than the cross section of the free molecule, which would be reduced then to $\simeq 7.2 \times 10^{-29} \mathrm{~cm}^{2} / \mathrm{sr}$. The latter value is the one we use later when determining the SERS enhancement factors (see Section 5.5.3) as the SERS experiments were carried out in air.

It is worth mentioning at this stage that a value of the cross section $\left.\frac{d \sigma}{d \Omega}\right)_{633 \mathrm{~nm}}^{\left(A_{g}(2)\right)}=7.2 \times 10^{-29} \mathrm{~cm}^{2} / \mathrm{sr}$ is only a factor of $\simeq 10$ smaller than typical cross sections for fingerprint modes of typical pre-resonant dyes used in SM-SERS. For example, the differential cross section for the $610 \mathrm{~cm}^{-1}$ mode of rhodamine $6 \mathrm{G}(\mathrm{RH} 6 \mathrm{G})$ at $633 \mathrm{~nm}$ is $6.7 \times 10^{-28} \mathrm{~cm}^{2} / \mathrm{sr}$ [19], and single molecules of RH6G can be easily observed with this cross section with enhancement factors of the order of $\simeq 10^{8}$. Taking into account that maximum SERS enhancement factors can go up to $\simeq 10^{10}-10^{11}$, the characterization of the differential cross section of $\mathrm{C}_{60}$ 


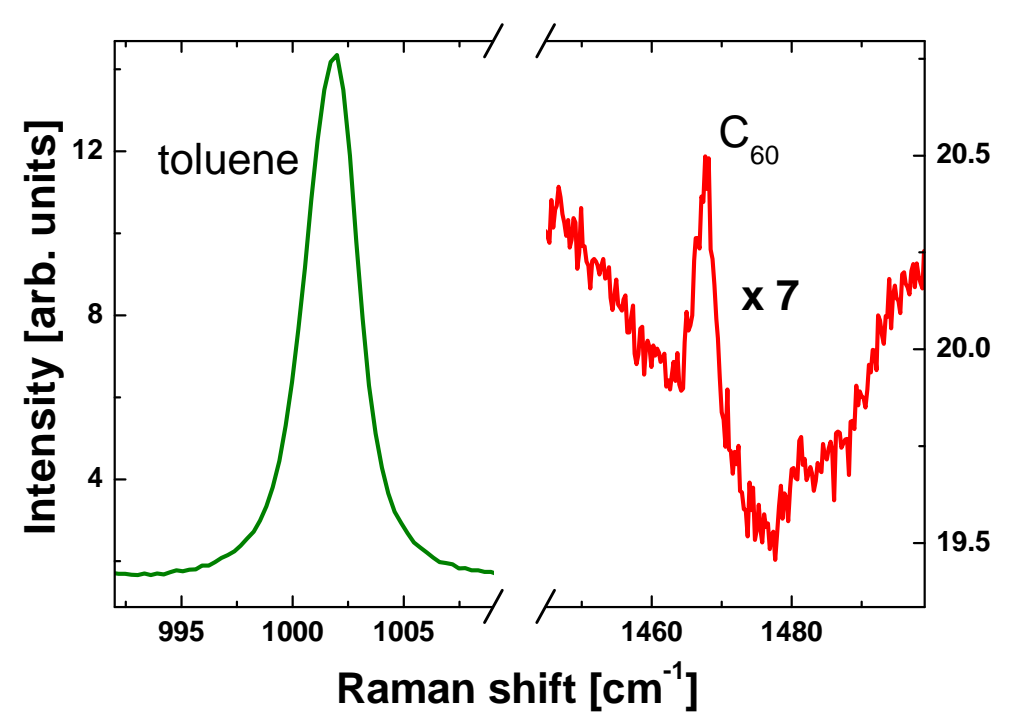

Figure 5.7: Determination of the differential Raman cross-section of the $1469 \mathrm{~cm}^{-1}$ mode.

immediately suggests that single molecules should be detectable; except for limitations related to the ability of $\mathrm{C}_{60}$ to reach the right places (hotspots) to profit from the enhancement, or additional factors linked to the photostability of the molecule [29].

\subsubsection{DFT calculation of the Raman spectrum of $\mathrm{C}_{60}$ and determination of the differential Ra- man cross-section of the $A_{g}(2)$ mode}

To double-check the accuracy of the experimental value of $\left.\frac{d \sigma}{d \Omega}\right)_{633 \mathrm{~nm}}^{\left(A_{g}(2)\right)}$, we use Density Functional Theory (DFT) calculations as a tool to predict the intensities of the Raman active modes of an (isolated) $\mathrm{C}_{60}$ molecule; the DFT calculated value of the cross section is only an estimate and doesn't suffice in itself but is a good basis of comparison when experimental cross sections are measured. Even though DFT is particularly suited for small molecules, we could assume that in the case of $\mathrm{C}_{60}$, despite its number of atoms, its high symmetry would help decrease the complexity of the normal mode problem and be less computationally demanding. 
To perform the DFT computations we used the software Gaussian $09^{2}$, which is dedicated to electronic structure modeling [113]. Starting from the fundamental laws of quantum mechanics, Gaussian 09 predicts the energies, molecular structures, vibrational frequencies and molecular properties using a wide variety of methods. The most stable geometry structure of a molecule is obtained by minimizing the total energy as a function of atoms' positions. The DFT approach consists in solving and finding the ground state of the Kohn-Sham equation [114]:

$$
\left[-\frac{\hbar^{2}}{2 m} \nabla^{2}+V_{\mathrm{KS}}(n(\mathbf{r}), \mathbf{r})\right] \Psi(\mathbf{r})=E \Psi(\mathbf{r})
$$

where $V_{\mathrm{KS}}$ is the Kohn-Sham potential which depends on the electronic density:

$$
n(\mathbf{r})=|\Psi(\mathbf{r})|^{2}
$$

hence $V_{\mathrm{KS}}$ is a function of the electronic density. DFT replaces the complex solving of a many-body problem for the electrons by a simpler problem of solving a one-electron Shrödinger equation in a non-linear potential depending on the electronic density.

The wave-function solution $\Psi(\mathbf{r})$ is a expressed as a linear combination of basis functions $\phi_{i}(\mathbf{r})$ called the basis set. The form of the Kohn-Sham potential (its dependence on $n(\mathbf{r})$ ) has to be chosen as an initial guess by the user as well as the basis set to be used to express the wave-function solutions; these choices are crucial and depend on the system being studied, the computational power available, etc. The first density functional perturbation theory calculation on $\mathrm{C}_{60}$ was performed in 1994 [115] and in 2001, Schettino et al. have shown that a DFT B3LYP/6-31G* calculation of the vibrational frequencies of $\mathrm{C}_{60}$ grants a very small $\left(\approx 3.6 \mathrm{~cm}^{-1}\right)$ average misfit between the experimental and calculated frequencies [116]. We used Beckes 3-parameter hybrid functional B3LYP, which employs a hybrid of Becke and Hartree-Fock exchange energy with a combination of VWN and LYP local and non-local correlation energy [117, 118, 119, 120] and the basis set designated as $6-311 \mathrm{G}(\mathrm{d})$ which is a set of Gaussian-like

\footnotetext{
${ }^{2}$ Official Gaussian website: www .gaussian.com
} 
orbitals [121]. The main limitations in the size and complexity of the basis set are most of the time based on computational time.

\section{Geometry optimization}

The first step is to find the equilibrium atomic positions, this is called geometry optimization. Vibrational analysis, as it is described in most textbooks and implemented in Gaussian 09 is valid only when the first derivatives of the energy with respect to atomic displacements are zero: in other words, the starting point of the analysis requires the molecule to be in its equilibrium position and this optimization has to be done with the same method and same basis set as the following vibrational analysis.

\section{Vibrational analysis: practical implementation}

The starting point of the treatment of vibrations in molecules is the BornOppenheimer approximation, in which the electronic cloud of a molecule adapts instantaneously to the configuration of the nuclei. Once the geometry optimization has been done, the next step is to calculate the force constant matrix or Hessian matrix.

The classical approach is sufficient to define the concept of normal modes if we introduce some parameters empirically. For a molecule with $N$ atoms indexed by $k=1 \ldots N$, each with a mass $m_{k}$, the coordinates of the nuclei can be expressed in terms of the displacements $\overrightarrow{u_{k}}$, representing their relative positions with respect to their equilibrium positions. Instead of the vectors $\overrightarrow{u_{k}}$, it can be convenient to use $3 N$ scalar variables accounting for all the Cartesian components of the $\mathrm{N}$ vectors $\xi_{1}=u_{1 x}, \ldots, \xi_{3 N}=u_{N z}$ and to divide them by the squared mass of the atom they relate to in order to get the reduced-mass coordinates:

$$
q_{i}=\sqrt{m_{i}} \xi_{i}
$$

The potential energy of the classical Hamiltonian for the nuclei, in 
the limit of small displacements of the nuclei, can be written:

$$
\begin{aligned}
V= & V_{0}+\sum_{i=1}^{3 N}\left(\frac{\partial V}{\partial q_{i}}\right)_{q_{i}=0} q_{i}+\frac{1}{2} \sum_{i, j=1}^{3 N}\left(\frac{\partial^{2} V}{\partial q_{i} \partial q_{j}}\right)_{q_{i}, q_{j}=0} q_{i} q_{j} \\
& +\frac{1}{6} \sum_{i, j, k=1}^{3 N}\left(\frac{\partial^{3} V}{\partial q_{i} \partial q_{j} \partial q_{k}}\right)_{q_{i}, q_{j}, q_{k}=0} q_{i} q_{j} q_{k}+\ldots
\end{aligned}
$$

where $V_{0}$ can be chosen to be zero and the second term, corresponding to the sum of the forces on the nuclei must also be zero as the atoms are in their equilibrium position. Keeping only the lowest order (harmonic approximation),

$$
V=\frac{1}{2} \sum_{i, j=1}^{3 N} f_{i, j} q_{i} q_{j}, \text { where } f_{i, j}=\left(\frac{\partial^{2} V}{\partial q_{i} \partial q_{j}}\right)_{q_{i}, q_{j}=0}
$$

The $f_{i, j}$ are called the force constants and $\left(f_{i, j}\right)$ is the force constant matrix and is real and symmetric; hence is diagonalizable. The way Gaussian 09 determines $\left(f_{i, j}\right)$ is by displacing each atom, one at a time, along three mutually perpendicular axis from their equilibrium position and recalculating the total energy of the new configuration by a new DFT calculation, then by numerical differentiation, the force constant matrix can be constructed.

Gaussian 09 then diagonalizes the force constant matrix which yields a set of $3 N$ eigenvectors and $3 N$ eigenvalues but stores only the eigenvalues (frequencies squared) and discards the eigenvectors which will be recalculated later, once the translational and rotational modes are separated out (by finding a matrix which diagonalizes the moment of inertia tensor, it will enable the generation of the vectors corresponding to rotations and translations). Then the software determines the transformation from the $3 N$ reduced-mass Cartesian coordinates to $3 N$ coordinates in the rotating and translating frame (internal coordinates), a frame in which the translations and rotations of the molecule are separated from the remaining $3 N-6$ vibrational modes.

The Hessian matrix $\left(f_{i, j}\right)$ in the reduced-mass coordinates is then trans- 
formed into the new internal coordinates $\left(f_{\text {int }}\right)$. The restriction of $\left(f_{\text {int }}\right)$ to the coordinates corresponding only to vibrations is then diagonalized, yielding $3 N-6$ eigenvalues $\omega^{2}$ and $3 N-6$ eigenvectors.

\section{Raman activities and depolarization ratios}

Gaussian 09 provides the Raman activities $R_{k}$ (from which are inferred the differential Raman cross-sections out of resonance) and Raman depolarization ratios $\rho_{k}$ for each normal mode. To get the Raman activities, Gaussian has to evaluate the Raman tensor. First the linear optical polarizability tensor of the molecule is calculated using DFT by recomputing the electronic structure of the molecule in the presence of an external static field. Then the linear polarizability tensor is re-evaluated for the molecule undergoing the deformations that follows the pattern of each of the normal modes of vibration. The difference between the linear polarizability tensor of the molecule with deformation and the linear polarizability tensor without deformation yields the Raman tensor $\hat{R}_{k}$. From $\hat{R}_{k}$ it is possible to calculate the absolute differential Raman cross-section (at room temperature) using the equation Eq. 5.15 [1], (and being careful with the units as Gaussian 09 typically gives the Raman activities in units of $\left.\left[\AA^{4} / \mathrm{u}\right]\right)$ :

$$
\begin{aligned}
\frac{d \sigma_{k}}{d \Omega}\left[\mathrm{cm}^{2} / \mathrm{sr}\right]= & 5.8 \times 10^{-46} \frac{\left[10^{7} /\left(\lambda_{\mathrm{L}}[\mathrm{nm}]\right)-\left(\bar{\nu}_{k}\left[\mathrm{~cm}^{-1}\right]\right)\right]^{4}}{\left(\bar{\nu}_{k}\left[\mathrm{~cm}^{-1}\right]\right)} \\
& \times L_{M} \times\left(R_{k}\left[\AA^{4} / \mathrm{u}\right]\right) \times\left(1-\exp \left(-\frac{\bar{\nu}_{k}\left[\mathrm{~cm}^{-1}\right]}{201.56}\right)\right)^{-1}
\end{aligned}
$$

where $\lambda_{L}$ is the wavelength of the laser, $\bar{\nu}_{k}$ the wavenumber of the mode under consideration and $L_{M}$ the local field correction factor presented earlier accounting for the presence of the solvent (toluene). For the $A_{g}(2)$ mode of $\mathrm{C}_{60}$ at $1469 \mathrm{~cm}^{-1}$ with an excitation $\lambda_{L}=633 \mathrm{~nm}$ and $L_{M} \simeq 4$, Eq. 5.15 gives:

$$
\left.\frac{d \sigma_{\mathrm{DFT}}}{d \Omega}\right)_{633 \mathrm{~nm}}^{\left(A_{g}(2)\right)}=3.81 \times 10^{-29} \mathrm{~cm}^{2} / \mathrm{sr}
$$


The theoretical value is a factor of $\sim 7$ smaller than the experimental one $\left.\left(\frac{d \sigma_{e x p}}{d \Omega}\right)_{633 \mathrm{~nm}}^{\left(A_{g}(2)\right)}=2.9 \times 10^{-28} \mathrm{~cm}^{2} / \mathrm{sr}\right)$, but this can be easily accounted for by an additional resonant enhancement (which has not been included in the calculation). It is well known that $\mathrm{C}_{60}$ has vibronic absorptions in the visible range [122], and these can easily contribute to a mild resonant enhancement for the cross sections compared to calculations. We use therefore the more reliable experimentally determined value for the estimation of SERS enhancement factors in Section 5.5.3.

\subsubsection{Resolution of the isotopically induced frequency shifts on $\mathrm{C}_{60}$ at $9 \mathrm{~K}$}

As one of the preliminary experiments, we wanted to know whether we could resolve the frequency shifts due to isotopic substitution in the $\mathrm{C}_{60}$ sample with a natural ${ }^{13} \mathrm{C}$ abundance in an experiment similar to the low temperature one conducted by Guha et al. [97]. A closed cycle Helium cryostat is adapted to our T 64000 spectrometer, enabling scattered light from a sample in the cryostat chamber to enter directly the spectrometer via the first entrance slit (the excitation and collection are not done through the microscope). This cryostat can cool samples down to $\simeq 5 \mathrm{~K}$.

The commercial natural $\mathrm{C}_{60}$ powder was dissolved in toluene (freezing point at $180 \mathrm{~K}$ ) with a final concentration of $1.05 \mathrm{mM}$. We enclosed $100 \mu \mathrm{L}$ of this solution within the tip of a glass pipette which was then carefully broken and sealed at both ends with Teflon tape. The Raman measurements were done on the T64000 spectrometer with the $647 \mathrm{~nm}$ excitation line of the Ar-Kr laser, the $1800 \mathrm{l} / \mathrm{mm}$ gratings. The scattered light was collected in a $90^{\circ}$ configuration and focused through through a $\times 10$ macro-lens onto the entrance slit of the spectrometer. As the signal in the additive mode (which we usually use) was too weak, we switched to subtractive mode and closed the slits (first entrance and intermediate) to a minimum. To adjust the slit size (and thus the resolution of the peaks), we were monitoring the resolution and intensity of the $1002 \mathrm{~cm}^{-1}$ mode of toluene in real time at room temperature and then all the way through the cooling procedure until the sample temperature was $9 \mathrm{~K}$ and the 


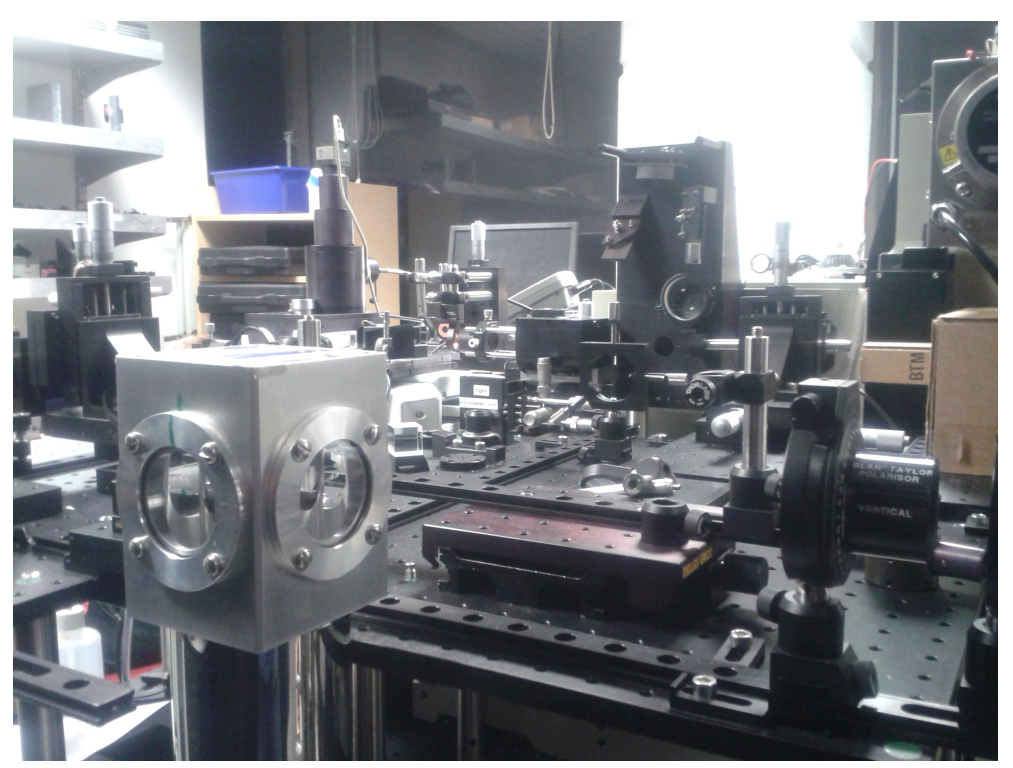

Figure 5.8: Closed cycle He cryostat and entrance to the T64000

Raman signal of the toluene was stable with a peak width of $\simeq 1 \mathrm{~cm}^{-1}$. To be able to resolve this narrow linewidth was a good indication that the isotopic splitting of the $1469 \mathrm{~cm}^{-1}$ mode of $\mathrm{C}_{60}$ would be detectable. The spectrum in Fig. 5.9 shows the results for a 3 hour long integration.

By freezing the $\mathrm{C}_{60}$ molecules into a solid transparent matrix at low temperature, we reduce additional contributions to the broadening of the peak and a substructure of 'shoulders' coming from the different natural isotopic versions of $\mathrm{C}_{60}$ can be revealed. The green, red, and blue curves in Fig. 5.9 are the three components of the best fit to the data. For the fit we proceeded as follows: the frequency of the pure ${ }^{12} \mathrm{C}_{60}$ was allowed to vary but the frequency positions of the other two peaks were to be in a fixed relation to this one, according to Eq. 5.3. The relative intensity ratios of the peaks is fixed in the ratio: $0.5150: 0.3437: 0.1128$, which is the probability of picking 60 atoms with a probability of 0.989 , the probability of picking 59 atoms with a probability of 0.989 times the probability of picking one ${ }^{13} \mathrm{C}$ with a probability of 0.011 (times 60 ) and the probability of picking 58 atoms with a probability of 0.989 times the probability of picking two ${ }^{13} \mathrm{C}$ (with $\mathrm{p}=0.011$ ) times $(60$ ! $/ 58$ !2!). So the only parameter allowed to vary was the width of the peaks (chosen to be 


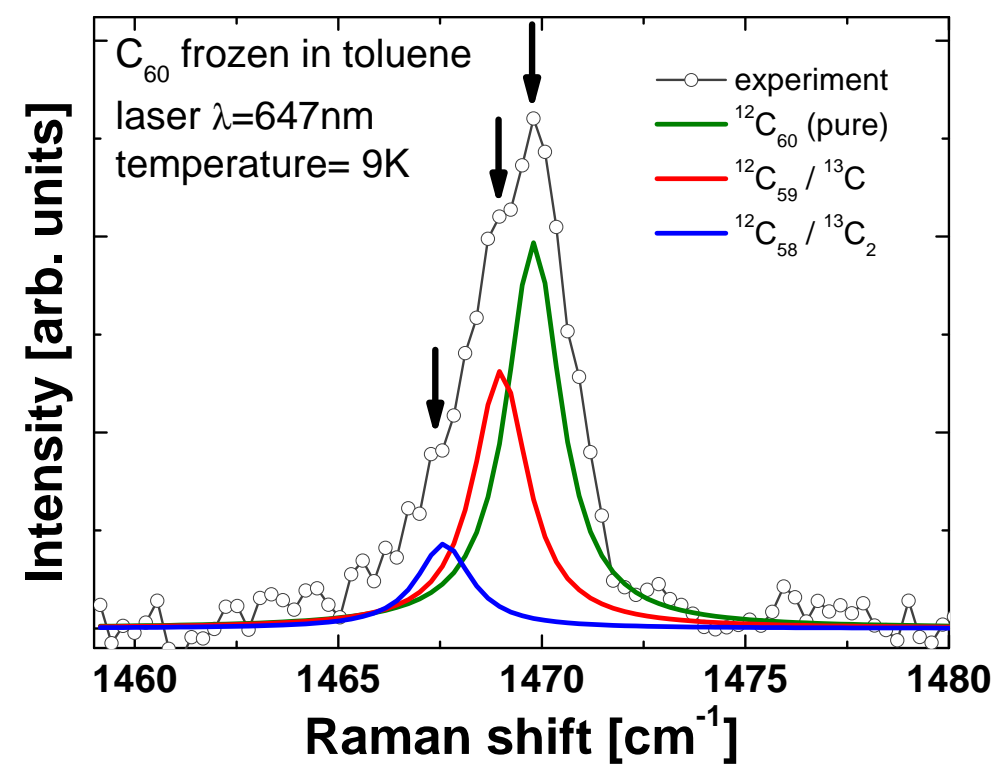

Figure 5.9: Low temperature (9 K) Raman spectrum of the pentagonal pinch mode of natural $\mathrm{C}_{60}$ frozen in toluene (647 $\mathrm{nm}$ excitation line). The presence of one and two ${ }^{13} \mathrm{C} \longrightarrow{ }^{12} \mathrm{C}$ isotopic substitutions can be seen as shoulders to the main peak of pure ${ }^{12} \mathrm{C}_{60}$ at $1469 \mathrm{~cm}^{-1}$. Due to its symmetry properties, the pentagonal pinch mode is isomorphic to the mass spectrum. 
the same for all three peaks) and an overall intensity scaling factor. We obtain a width of $0.8 \mathrm{~cm}^{-1}$ for the peaks. The implicit approximation we used here is to assume it does not matter which ${ }^{12} \mathrm{C}$ is replaced, the effect on the frequency shift of the $A_{g}(2)$ mode is the same, which is justified in Section 5.3 and in [110].

Can we use the natural isotopic effect of the $A_{g}(2)$ mode to detect SM-SERS of $\mathrm{C}_{60}$ ?

Unfortunately, the natural isotopic effect in $\mathrm{C}_{60}$ cannot be used for single molecule SERS detection. The reason is that the separation among peaks is only $\sim 1 \mathrm{~cm}^{-1}$ as we just saw above. This small shift competes with the intrinsic broadening of the peaks -which is $\sim 1.5-2 \mathrm{~cm}^{-1}$ (even at very low temperatures, as in Fig. 5.9) and the additional small frequency variations produced by the environment under SERS conditions (which is the origin of the inhomogeneous broadening studied in Chapter 4). The combination of the two effects washes out our ability to easily distinguish single isotopic versions of $\mathrm{C}_{60}$ differing by one mass unit.

A much easier strategy to detect SM-SERS of $\mathrm{C}_{60}$ is, in fact, to increase the isotopic spread of the natural sample to make the different isotopic versions of the molecule more easily distinguishable despite the intrinsic homogeneous broadening of the peaks and the possible addition of small frequency shifts from the environment.

\subsection{SERS and single-molecule SERS detec- tion of $\mathrm{C}_{60}$}

The first SERS studies on $\mathrm{C}_{60}$ on noble metal were published in 1991 and since then some work has been done on SERS, mainly as a way to probe the interactions between fullerenes and substrates [123, 124, 125, 126]. On our route to single molecule detection of $\mathrm{C}_{60}$, we started by carrying out preliminary experiments with the natural $\mathrm{C}_{60}$ sample to understand how the molecule was behaving in SERS conditions, what 
kind of substrate would be appropriate and how low in concentration we could work.

\subsubsection{SERS experiments on natural $\mathrm{C}_{60}$}

\section{The quest for a suitable SERS substrate}

The preparation of SERS samples with $\mathrm{C}_{60}$ was somewhat challenging and time-consuming. One of the obvious challenges is the fact that $\mathrm{C}_{60}$ is not water-soluble and the colloids we use routinely in our group are dispersed in water and thus the mixing of the $\mathrm{C}_{60}$ in solution with colloids before dropcasting onto a planar surface is not as straightforward as it is with Nile blue for example. Hence we tried silver films: commercial $50 \mathrm{~nm}$ Ag films roughened by exposure to ambient air, island films that we evaporated on glass slides and thick $(\sim 4 \mu \mathrm{m})$ silver films prepared also by thermal evaporation and then roughened by exposure to a $\mathrm{Ar}^{+}$plasma. We tested the SERS efficiency of the evaporated films with rhodamine $6 \mathrm{G}$, which showed the island films to be the only SERS active substrates. However, any silver film was deteriorated almost instantaneously when $\mathrm{C}_{60}$ dissolved in toluene or dichloromethane was deposited. The same deterioration was observed when trying to deposit drops of $\mathrm{C}_{60}$ solution in toluene onto dried Lee \& Meisel colloids.

Eventually, we found out a suitable way of preparing SERS and SMSERS samples and this is the sample preparation which we used in the following experiments: freshly prepared borohydride reduced silver colloids (see Section 2.3.1) were centrifuged in four eppendorfs for $5 \mathrm{~min}$ at $14.5 \times 10^{3} \mathrm{rpm}$, the supernatant water was sucked off, thus leaving concentrated colloids at the bottom. We then dropcast a few drops of these colloids onto a commercial $100 \mathrm{~nm}$ silver film and left it to dry out under a lamp for an hour. The resulting substrate is covered by clusters of colloids and can be expected to have many hots spots.

We obtained the first satisfying SERS results on $\mathrm{C}_{60}$ with this substrate: $\mathrm{C}_{60}$ is dissolved in $\mathrm{CH}_{2} \mathrm{Cl}_{2}$ (solubility $0.26 \mathrm{mg} / \mathrm{mL}$ ) with $20 \mu \mathrm{M}$ concentration, as $\mathrm{CH}_{2} \mathrm{Cl}_{2}$ did not seem to deteriorate the silver substrate. Then the solution was dropcast onto the substrate drop by drop, letting 
each drop evaporate before putting the next one and covering this way the whole surface of dried colloids. Fig. 5.10 shows some SERS spectra around the region of the $A_{g}(2)$ mode taken on the latter sample in the Linkam cryostat at $80 \mathrm{~K}$ with $633 \mathrm{~nm}$ excitation $(2.5 \mathrm{~mW}$ on the sample), the $\times 100$ LWD objective, the $2400 \mathrm{l} / \mathrm{mm}$ gratings, the confocal pinhole closed to $200 \mu \mathrm{m}$ and $1 \mathrm{~s}$ acquisition. It is worth noticing at this point that out of a map of the sample of around 2500 spectra, only $1-2 \%$ were identified as showing the $A_{g}(2)$ mode peak. By reducing the incident power by a factor of 10 , we increased slightly more the number of detectable events $(3-4 \%)$, showing thus that $\mathrm{C}_{60}$ is photosensitive and it is better to work with an incident power $\sim 250 \mu \mathrm{W}$. We also carried out the same experiment as before but with a starting solution of $\mathrm{C}_{60}$ $2 \mathrm{M}$ in $\mathrm{CH}_{2} \mathrm{Cl}_{2}$ and got a similar statistics of SERS events.

These SERS experiments however confirmed what we already suspected, i.e. that the natural isotopic effect can not be used for Single-Molecule SERS detection. Eq. 5.3 indeed predicts a shift of the frequency of the $A_{g}(2)$ mode of $\sim 1 \mathrm{~cm}^{-1}$ for each ${ }^{13} \mathrm{C} \longrightarrow{ }^{12} \mathrm{C}$ substitution and this competes with the natural linewidth of the peak $\left(\sim 2 \mathrm{~cm}^{-1}\right.$ at $\left.80 \mathrm{~K}\right)$, even at $80 \mathrm{~K}$, and the small frequency shifts induced by the interaction of the molecules with the surface.

\section{Tentative bi-analyte experiments on $\mathrm{C}_{60}$ and $\mathrm{BPE}$}

It is possible to some degree to find a bi-analyte SERS [25] partner for $\mathrm{C}_{60}$; and we performed experiments with $\mathrm{C}_{60}$ and 1,2-di-(4-pyridyl)ethylene (BPE) in which we were able to distinguish individual spectra of either one compound or the other. However, the bi-analyte method with two different partners works at its best when the molecules have similar chemical properties and comparable affinities for the SERS-active surface. The unique properties of $\mathrm{C}_{60}$ in terms of size, shape, and chemistry, makes it difficult to find a suitable partner; and experiments with BPE had to be performed at widely different concentrations for the two molecules (to compensate for the different surface affinity properties). This leads to very skewed statistics of SM-events, in which the two partners might not be experiencing the same enhancement factor distribu- 


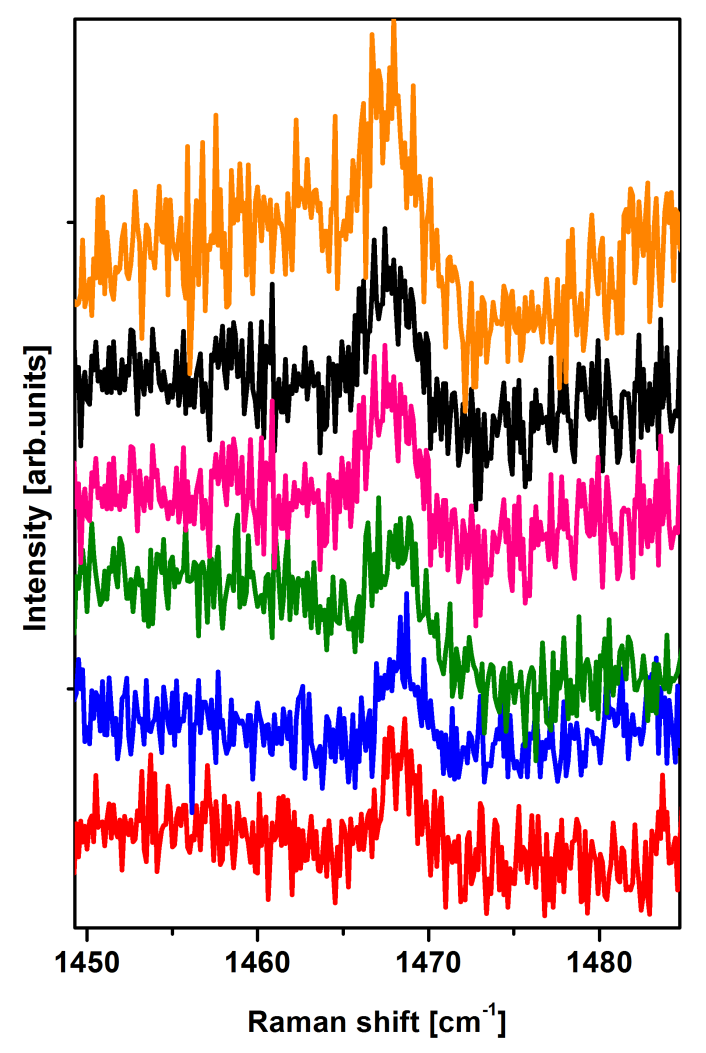

Figure 5.10: High resolution SERS spectra around the $1469 \mathrm{~cm}^{-1}$ mode of natural $\mathrm{C}_{60}$ dried on borohydride reduced silver colloids at $80 \mathrm{~K}, 633 \mathrm{~nm}$ excitation and $1 \mathrm{~s}$ acquisition. Note that it is not possible to ascertain whether these spectra are single molecule spectra relying solely on natural isotopically induced frequency shifts as for natural $\mathrm{C}_{60}$, only shifts of $1 \mathrm{~cm}^{-1}$ (1 substitution) or $2 \mathrm{~cm}^{-1}$ (2 substitutions) are statistically expected and these shifts compete with the broadening and frequency wandering of the peak due to SERS conditions.

tions.

As the bi-analyte method was not successful and as the spread in frequencies of the $A_{g}(2)$ mode of the natural $\mathrm{C}_{60}$ molecule was not sufficient with respect to the intrinsic broadening of the peak to allow for 
discrimination of SM events by frequency wandering only, we opted for the use of artificially enriched $\mathrm{C}_{60}$ for the following SM-SERS studies.

\subsubsection{Experiments on $\mathrm{C}_{60}$ commercial isotopologues}

Isotopically enriched $\mathrm{C}_{60}$ with an average of 18 sites replaced by ${ }^{13} \mathrm{C}$ was obtained commercially (MER Corp.Tucson, AZ) and characterized by high-performance liquid chromatography (HPLC), and mass spectrometry (seen in Fig. $5.11(\mathrm{a})$ ). The most probable atomic mass in this sample is $m=738$; which implies that 18 carbons (30\% of the total) have been replaced by ${ }^{13} \mathrm{C}$. But there are (at least) 14 different isotopic variations around this most probable case, as shown in Fig. 5.11 (a); from $m=732$ to 745 .

We can use the mass spectrum in Fig. 5.11(a) as a probability distribution and calculate the histogram of possible frequencies for a large number of $\mathrm{C}_{60}$ molecules where the isotopic replacement sites are chosen at random. This is done with the force constants calculated by DFT in the computation we made before to obtain the Raman cross section of the pentagonal pinch mode (see Section 5.4.2). The DFT force constant matrix $\left(f_{i, j}\right)$ does not depend on the masses of the atoms, and can be used therefore for any combination of isotopically substituted $\mathrm{C}_{60}$ with randomly chosen sites for ${ }^{13} \mathrm{C}$, and a mass distribution given by the experimentally determined values in Fig. 5.11 (a). This calculation is shown in Fig. 5.11 (b) for $10^{4}$ different isotopic versions of $\mathrm{C}_{60}$.

Note that:

* Frequencies in the calculation are overestimated with respect to experimental values (a well known fact for DFT [1, 15])

* The histogram of calculated frequencies displays a series of discrete peaks mimicking the mass distribution. This does not happen for other vibrational modes in the molecule and it is again a manifestation of the peculiar symmetry properties of the pentagonal pinch mode and the fact that ${ }^{13} \mathrm{C}$ isotopic disorder (for a fixed number of ${ }^{13} \mathrm{C}$ ) does not play a noticeable effect in the overall frequency shift 

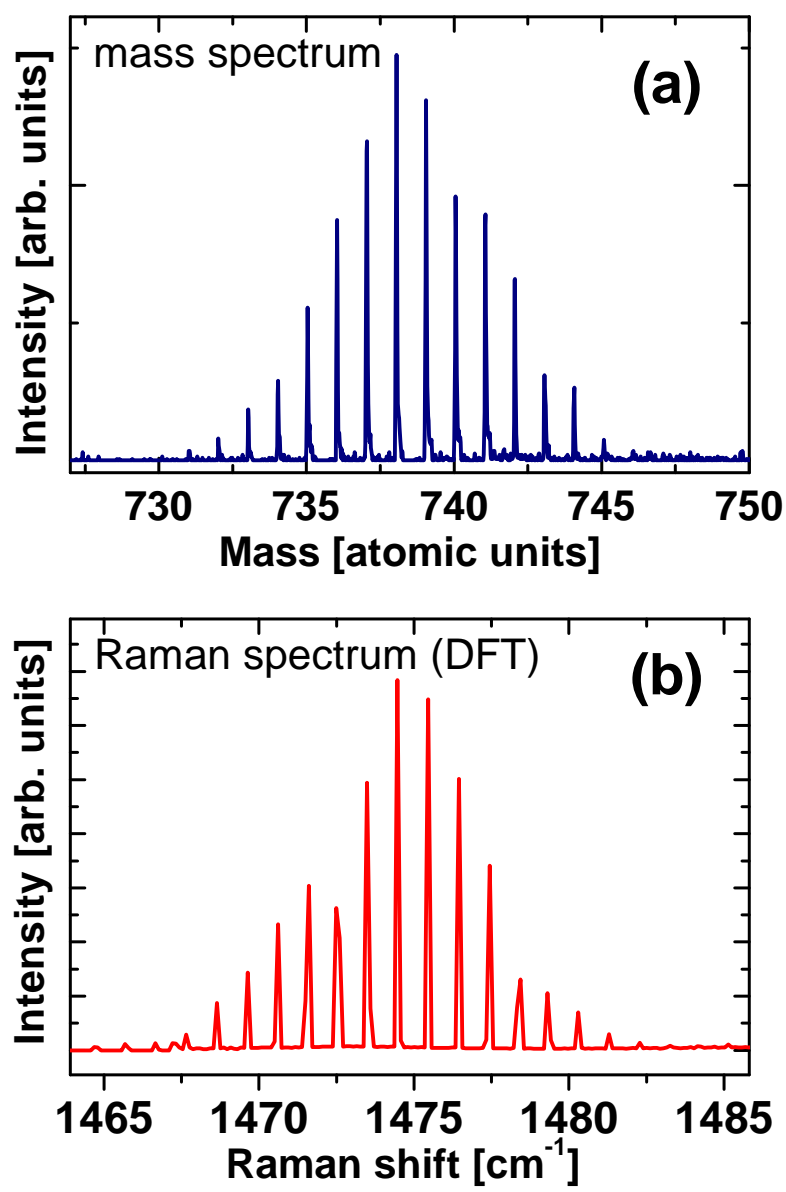

Figure 5.11: (a) Experimental mass spectrum of the isotopically substituted (30\% average) sample used for SM-SERS. There are (at least) 14 different isotopic substitutions around the average of $30 \%$ in the sample. (b) Vibrational Raman spectrum calculated from the DFT force constants for $10^{4}$ random versions of isotopically substituted $\mathrm{C}_{60}$ chosen with a probability distribution given by the mass spectrum in $(a)$. Because of the symmetry of the pentagonal pinch mode, the Raman spectrum is isomorphic to the mass distribution, resulting in a series of discrete frequencies mimicking the mass spectrum (see Section 5.3). The Raman frequencies are overestimated in DFT calculations, which is why the full spectrum appears at slightly larger frequencies than those observed experimentally in Fig. 5.12.

(which depends then only on the total mass $M$ ). Furthermore, since the magnitude of the Raman cross section for individual isotopic versions of $\mathrm{C}_{60}$ is an electronic property that does not depend on small mass-induced shifts of the frequency, the histogram 
of frequencies in Fig. 5.11 (b) automatically represents the Raman spectrum of the ensemble of all possible isotopic versions of $\mathrm{C}_{60}$ contained in the mass spectrum of Fig. 5.11 (a).

From the point of view of SM-SERS, the separation amongst consecutive peaks in the calculated spectrum of Fig. 5.11 (b) is still small $\left(\sim 1 \mathrm{~cm}^{-1}\right)$, and might still compete to be distinct enough when the natural linewidths of the peaks and the possible effects of small frequency shifts produced by the interaction with the surface [127] are taken into account. However, the existence of multiple peaks spanning a broad frequency range $\left(\sim 20 \mathrm{~cm}^{-1}\right)$ gives now the opportunity to identify different isotopic versions of $\mathrm{C}_{60}$ in SM-SERS; in what is a natural extension of the bi-analyte SERS technique [21] to multiple (isotopic) analytes.

SM-SERS experiments were carried out for the isotopic $\mathrm{C}_{60}$ sample excited at $633 \mathrm{~nm}$ and with a focus on high-resolution measurements of the $A_{g}(2)$. The SERS substrate we used is a variation of the one presented in Section 5.5.1. Concentrated (by centrifugation) borohydride reduced silver colloids were dropcast onto poly-L-lysine covered Si wafers (see Section 2.3.2), and allowed to dry under a lamp, thus leaving a dense collection of clusters on the surface. Small amounts of the isotopic $\mathrm{C}_{60}$ sample - previously dissolved in $\mathrm{CH}_{2} \mathrm{Cl}_{2}$ at $1 \mathrm{M}$ concentrationwere dropcast on top of the colloids and allowed to quickly evaporate. Compared to typical concentrations used in SM-SERS experiments for resonant dyes [127], the $\mathrm{C}_{60}$ solution is very concentrated. This is necessary to compensate for the much smaller affinity of $\mathrm{C}_{60}$ to stick to $\mathrm{Ag}$ colloids and find its place at hot spots on the substrate. The reasonably large number of colloid clusters achieved by centrifugation together with the much higher concentration of $\mathrm{C}_{60}$ compared to the dye ensures that SM-SERS conditions will be found in a few selected places in the sample, as demonstrated later.

Raman spectra were acquired using the $\times 100$ LWD $(\mathrm{NA}=0.6)$ with excitation at $633 \mathrm{~nm}(2.5 \mathrm{~mW}$ on the sample $)$ and the high resolution $1800 \mathrm{l} / \mathrm{mm}$ grating. The sample is placed inside the cryostat stage for optical microscopy, which keeps the sample at $80 \mathrm{~K}$ under a $\mathrm{N}_{2}$ atmosphere. SM-SERS maps of $40 \times 40$ points $(1.5 \mu \mathrm{m}$ step and $1 \mathrm{~s}$ integration time 


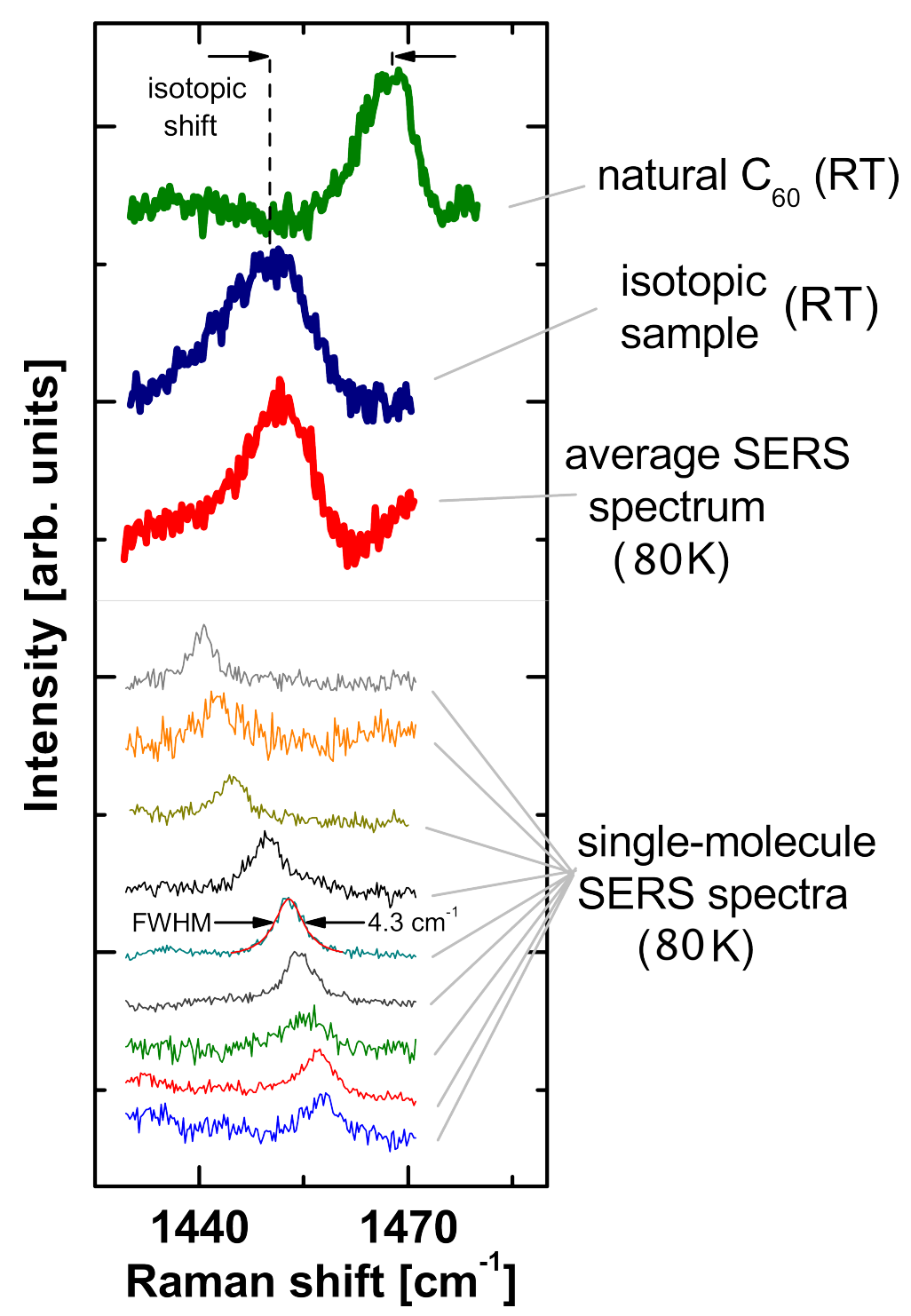

Figure 5.12: Room temperature Raman spectra of natural $\mathrm{C}_{60}$ powder (green) and isotopically enriched $\mathrm{C}_{60}$ powder (blue) and average SERS spectra of the isotopic sample at $80 \mathrm{~K}$. The average SERS spectrum shows a slightly narrower peak produced by lower temperature in which the search for SM-SERS signal is carried out. The bottom part of the figure shows different examples of SM-SERS signals within the frequency range spanned by the average $\left(\sim 20 \mathrm{~cm}^{-1}\right)$. For plotting purposes the SM-spectra have been normalized to show similar intensities, but they represent in reality signals with different intensities spanning enhancement factors in the range $6.5 \times 10^{7}-1.2 \times 10^{8}$. In addition, SM-SERS spectra have typical broadenings in the range of $\sim 4-5 \mathrm{~cm}^{-1}$, which are larger than the broadening observed in SM-spectra of natural $\mathrm{C}_{60}$ at the same temperature. This is attributed to isotopic-disorder induced broadening. 
per point) were taken to search for single-molecule $\mathrm{C}_{60}$ signals of different isotopic compositions. At least ten maps were required to obtain the necessary statistics of cases, as each map contained only $\sim 1 \%$ of clearly distinguishable SM-SERS spectra.

Fig. 5.12 summarizes the main SERS results. At the top of the figure we can observe the normal spectrum of natural $\mathrm{C}_{60}$ powder at room temperature (RT); note the slight broadening and asymmetry of the $A_{g}(2)$ peak caused by the presence of natural isotopic substitutions (although they are not resolvable as shoulders at RT, as they were at $9 \mathrm{~K}$ in Fig. 5.9). The powder of the isotopically substituted sample (30\%) at RT in Fig. 5.12 shows a peak with a maximum shifted to $1450 \mathrm{~cm}^{-1}$ which is in agreement with Eq.5.3), and a larger broadening than the natural sample (spanning $\sim 20 \mathrm{~cm}^{-1}$ ) produced by the different isotopic versions of the molecule. As with the natural sample, the individual isotopic versions cannot be distinguished due to the overlap and broadening of the individual peaks. The average SERS signal over $1.6 \times 10^{4}$ spectra at $80 \mathrm{~K}$ from the maps shows a distinct peak at approximately the same energy, but slightly narrower compared to room temperature (due to the sharpening effect of temperature on anharmonic contributions). It is worth noting that the inhomogeneous broadening of the peak in this case has its main contribution from the isotopic spread of the molecule, rather than interactions with the surface (which is the more classic contribution studied in Ref. [51]. By carefully going through the data it is possible to recover tens of single-molecule spectra per map. Examples of these spectra are given at the bottom of Fig. 5.12. The average of these sharp SM-SERS events for all the maps recovers the average spectrum, and they span in frequency over the entire $\sim 20 \mathrm{~cm}^{-1}$ range where SM-SERS spectra are expected. In Fig. 5.12 the SM-SERS spectra have been normalized in intensity, but in reality they span a range of intensities that can be quantified into a SERS differential cross section (by comparison to a standard), and can be transformed into a SERS enhancement factor (EF) with the help of the bare differential cross section; this will be the topic of the next section. In addition, the SM-SERS spectra show 
characteristic widths of $\sim 4-5 \mathrm{~cm}^{-1}$ and this turns out to be much broader (by a factor of $\sim 2$ ) compared to SM-SERS spectra of natural $\mathrm{C}_{60}$ on the experiments conducted before in Section 5.5.1 where most of the time a molecule of pure ${ }^{12} \mathrm{C}_{60}$ is detected. We attribute this interesting fact to an isotopic-disorder induced homogeneous broadening of the peak. Isotopic-disorder induced broadening is well known in crystals; including diamond which involves also combinations of ${ }^{12} \mathrm{C}$ and ${ }^{13} \mathrm{C}[128]$. In short, our interpretation is that the isotopic disorder is a second order correction for the frequency (which only depends on the total mass), but it becomes the leading correction for the broadening by "turning on" anharmonic interactions.

As a concluding remark, these SM-SERS experiments on isotopically enriched $\mathrm{C}_{60}$ reveal an interesting and unique aspect of spectroscopy. In the vast majority of cases, natural (and even artificial) isotopic effects have a very small contribution to the inhomogeneous broadening of a peak in SERS conditions, which is dominated by other environmental factors like the interaction with the surface as we have seen in Chapter 4 and Ref. [51] and in that framework it is possible to get from the statistics of signals a small minority of cases where two or three molecules are seen simultaneously within the inhomogeneous broadening of a peak. Hence, these minority signals represent the simultaneous observation of a few single molecules which are distinguished in the case of Ref. [51] by the different environmental conditions. In a way, the case at hand here is on the opposite extreme: the main contribution to the inhomogeneous broadening of the average SERS peak in Fig. 5.12 is the isotopic spread of the molecules. But except for that difference, the same phenomenology applies; i.e. it should be possible to observe more than one molecule simultaneously which are differentiated not so much by their environmental differences but rather by different isotopic compositions. This is shown explicitly in Fig. 5.13 where minority signals from two and three molecules can be observed. 


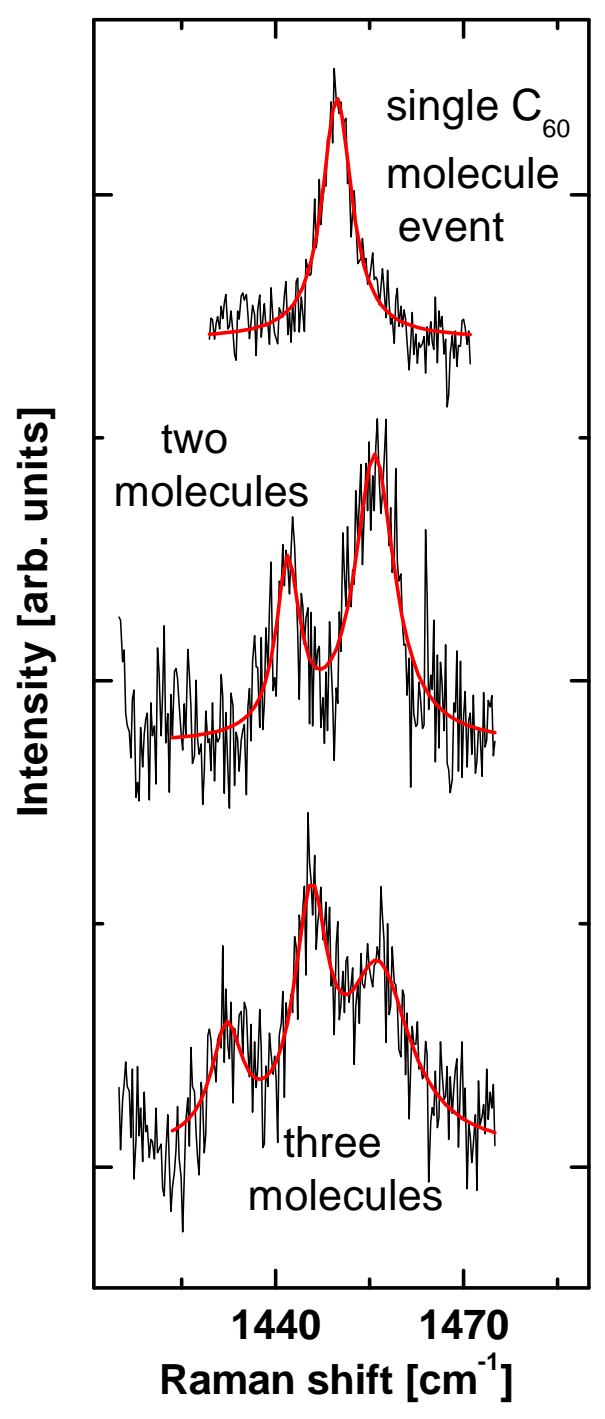

Figure 5.13: SERS spectra displaying cases with different number of molecules. Cases with one (top), two (middle) and three (bottom) molecules can be identified in SERS maps. Cases with two or three molecules are much rarer than single molecule ones, but can be eventually identified with sufficient sampling.

\subsubsection{Enhancement factors}

In addition to the discussion of the previous section, it is always worth evaluating the enhancement factors $(\mathrm{EF})$ for the observed SM signals to see whether they fall within an acceptable range with respect to what is known from electromagnetic theory [1]. Finding SM-EFs which would be 
orders of magnitude larger than $10^{8}-10^{9}$ would be indicative of many molecule spectra (the signal does not come from a single molecule but a large collection of them). The way to quantify them is to compare the integrated intensity of individual SM-SERS events with respect to a signal of known Raman differential cross section, under the same experimental conditions. This is be done by characterizing the effective scattering volume $V_{\text {eff }}$ of the objective and using nitrogen (in air) as a reference [19].

\section{Characterization of the $\times 100$ LWD objective scattering volume}

We have to compare the SERS intensity coming from one $\mathrm{C}_{60}$ molecule, ideally at the center of the focal plane, with the Raman intensity $I_{\text {ref }}^{\text {Tot }}$ coming from $N$ reference molecules at a concentration $c$ within the scattering volume $V_{\text {eff. }} \quad V_{\text {eff }}$ is defined as the volume from which the same Raman signal would be observed if the excitation (considered here to be Gaussian) and detection efficiency (of the spectrometer) were both uniform and equal to their maximum $I_{0}$ (intensity at the center of the focal plane) and $\eta_{0}$ (detection efficiency maximum when the scatterer is a point source at the center of the focal plane). For reference molecules with concentration $c$, The total Raman signal is:

$$
I_{\mathrm{ref}}^{\mathrm{Tot}}=c \mathcal{N}_{A} \sigma_{d} \int_{\rho=0}^{+\infty} 2 \pi \rho \int_{z=-\infty}^{+\infty} I(\rho, z) \eta(\rho, z) d \rho d z
$$

where $\sigma_{d}$ is the Raman cross section of the reference molecule over the whole detection solid angle $\Delta \Omega_{\text {det }}$ (determined by the numerical aperture of the objective), $z$ is the distance along the optical axis and $\rho$ is the radial distance from the optical axis.

$I(\rho, z)$ is the Gaussian excitation profile:

$$
I(\rho, z)=I_{0} \frac{w_{0}^{2}}{w(z)^{2}} \exp \left(-\frac{2 \rho^{2}}{w(z)^{2}}\right)
$$


where $w(z)$ is the spot size, $w_{0}$ is the waist of the Gaussian beam. The total power $P_{0}$ of the Gaussian beam is:

$$
P_{0}=\frac{\pi w_{0}^{2}}{2} I_{0}=A_{\text {eff }} I_{0}
$$

$A_{\text {eff }}$ is called the effective surface area of the Gaussian excitation.

The detection efficiency profile $\eta(\rho, z)$ depends mainly on the size and shape of the entrance slit. The dimension of the image on the entrance slit is related to the actual dimension of the sample by a factor $X$ (Objective magnification and other magnification parameters). For the Labram and a $\times 100$ objective, $X=56$.

$$
\eta(x, y, z)= \begin{cases}\eta_{\perp}(z) & \text { if }-L / X \leq x, y \leq L / X \\ 0 & \text { otherwise }\end{cases}
$$

where $\eta_{\perp}(z)$ is the axial detection efficiency, $L$ is the half dimension of the square pinhole. When the pinhole size is much larger than $w_{0} X$, which is easily the case when the pinhole is totally open (on the Labram this is $354 \mathrm{\mu m}$ ), we can neglect the effect of the lateral detection efficiency and we define an effective detection depth $H_{\text {eff }}$ such as:

$$
H_{\mathrm{eff}}=\int_{z=-\infty}^{z=+\infty} \frac{\eta_{\perp}(z)}{\eta_{0}} d z
$$

Eq. 5.17 then becomes:

$$
I_{\mathrm{ref}}^{\mathrm{Tot}}=c A_{\mathrm{eff}} H_{\mathrm{eff}} \mathcal{N}_{A} \sigma_{d} I_{0} \eta_{\mathrm{spec}} \frac{1}{1+\rho_{k}}
$$

where $\rho_{k}$ is the depolarization ratio and $\eta_{\text {spec }}$ is a factor describing the overall efficiency of the Labram spectrometer. To know the scattering volume of the $\times 100$ LWD objective, we need to know $H_{\text {eff }}$ and $A_{\text {eff }}$. To get $A_{\text {eff }}$, we measure $w_{0}$ by measuring the slit (pinhole) size dependence of the integrated intensity of the $520 \mathrm{~cm}^{-1}$ mode of $\mathrm{Si}$ with the $\mathrm{Si}$ sample being in the focal plane of the objective. The integrated intensity 
of the peak as a function of $L$ is given by:

$$
\begin{aligned}
\frac{I(L)}{I_{\max }}= & \frac{1}{4}\left[\operatorname{erf}\left(\frac{\sqrt{2}}{W_{0}}\left(x_{c}+L\right)\right)-\operatorname{erf}\left(\frac{\sqrt{2}}{W_{0}}\left(x_{c}-L\right)\right)\right] \\
& \times\left[\operatorname{erf}\left(\frac{\sqrt{2}}{W_{0}}\left(y_{c}+L\right)\right)-\operatorname{erf}\left(\frac{\sqrt{2}}{W_{0}}\left(y_{c}-L\right)\right)\right]
\end{aligned}
$$

where $x_{c}$ and $y_{c}$ are the coordinates of the center of the pinhole with respect to the origin (intersection between optical axis and pinhole) if the latter is slightly misaligned and $W_{0}=w_{0} X$.

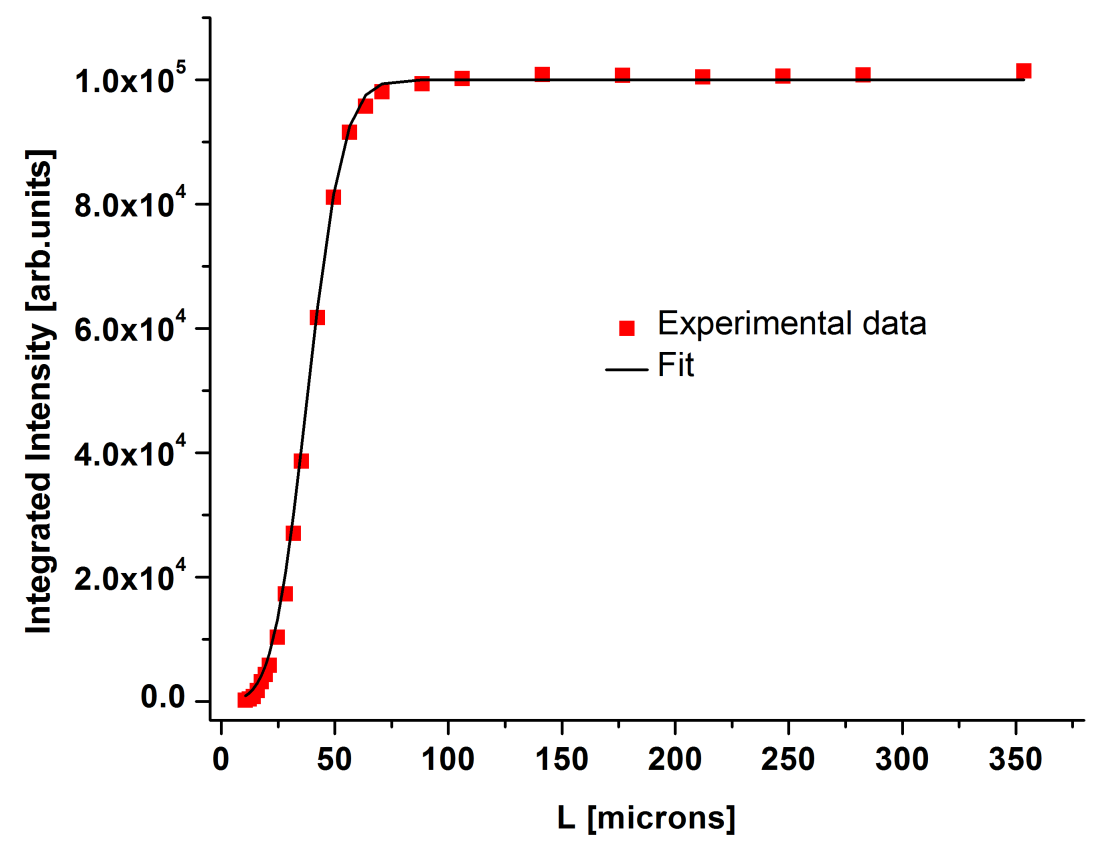

Figure 5.14: Slit size dependence of the integrated intensity of the $520 \mathrm{~cm}^{-1}$ of Si (red dots) and fit of the data according to Eq. 5.23 (black line).

Figure 5.14 shows the data from Si. The fit yields: $\left(x_{c}, y_{c}\right)=(30,30)$ in $\mu \mathrm{m}$ (the slit was slightly misaligned that day) and $W_{0}=28 \mu \mathrm{m}$, hence $w_{0}=0.5 \mu \mathrm{m}$. Then $A_{\mathrm{eff}}=0.392 \mu \mathrm{m}^{2}$.

To get $H_{\text {eff }}$ we measure the axial detection efficiency $\eta(z)$ by recording 
the integrated intensity of the $520 \mathrm{~cm}^{-1}$ peak of $\mathrm{Si}$ as a function of $z$ (focal depth) with the slit (pinhole) fully open. As the focusing adjustment knob on our system does not have a stepper motor fitted to it, we manually scanned through $120 z$ positions and recorded at each position the Raman spectrum around the $520 \mathrm{~cm}^{-1}$ mode. Fig. 5.15 shows the experimental data points. The pinhole being fully open:

$$
\begin{aligned}
H_{\text {eff }} & =\int_{-\infty}^{+\infty} \frac{\eta_{\perp}(z)}{\eta_{0}} d z \\
& =\int_{-\infty}^{+\infty} \frac{I(z, L)}{I_{\max }} d z \\
& =\frac{\sum_{i} I\left(z_{i}, L\right) \Delta z_{i}}{I_{\max }}
\end{aligned}
$$

Eq. 5.24 yields $H_{\text {eff }}=24 \mu \mathrm{m}$. Thus the scattering volume $V_{\text {eff }}$ for the $\times 100$ LWD objective is $V_{\text {eff }}=9.4 \mu^{3}$.

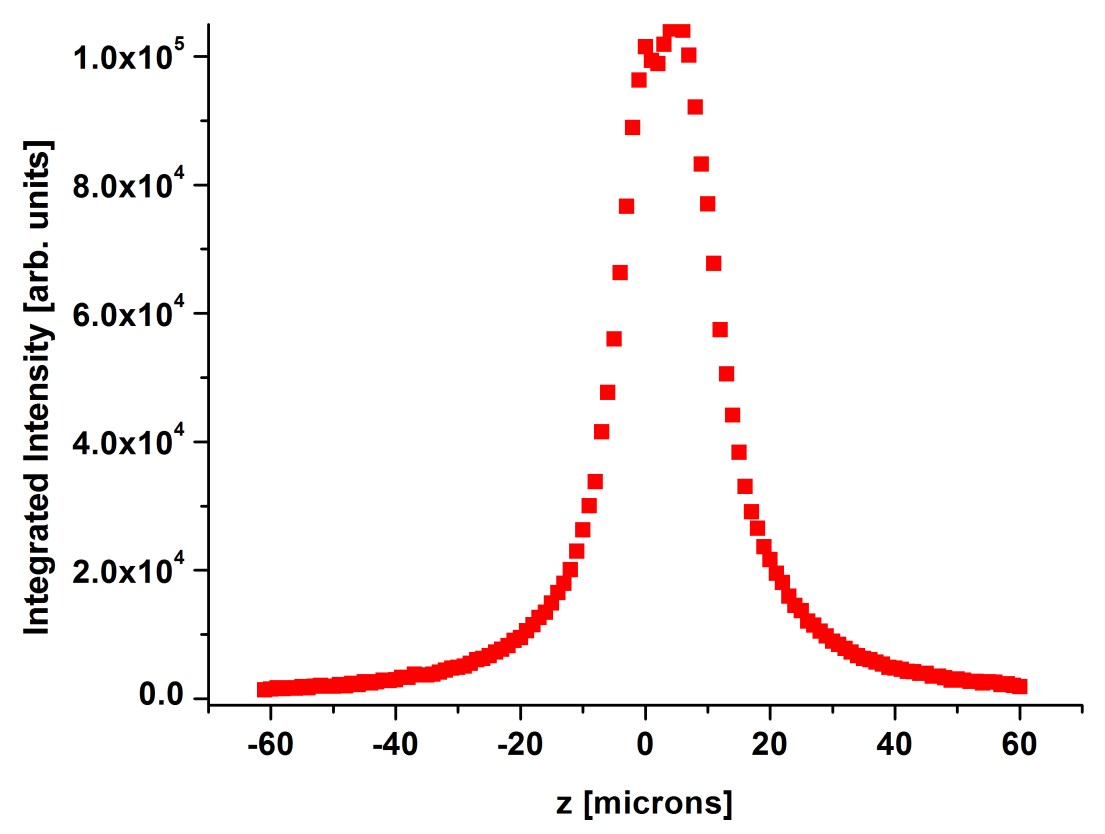

Figure 5.15: $z$ - dependence of the integrated intensity of the $520 \mathrm{~cm}^{-1}$ mode of Si with $\times 100$ LWD objective. 


\section{Single-molecule enhancement factors of the SM events of $C_{60}$}

There are $1.8 \times 10^{8}$ nitrogen molecules in $V_{\text {eff }}$ (at room temperature and normal pressure). Knowing that the $2230 \mathrm{~cm}^{-1}$ peak of $\mathrm{N}_{2}$ has a differential cross section (at $633 \mathrm{~nm}$ excitation) of $1.6 \times 10^{-32} \mathrm{~cm}^{2} / \mathrm{sr}$ [1], and normalizing by integration times with respect to the actual experiments in $\mathrm{C}_{60}$, we can put numbers to the SERS cross sections of individual single molecule events. Finally, by using the experimentally determined bare cross section for the pentagonal pinch mode $\left.\frac{d \sigma}{d \Omega}\right)_{633 \mathrm{~nm}}^{\left(A_{g}(2)\right)}=7.2 \times 10^{-29} \mathrm{~cm}^{2} / \mathrm{sr}$, we can transform the SERS cross sections into SERS enhancement factors (EFs). The EFs depend obviously on the specific SM-SERS event we are analyzing. The enhancement factors for the cases shown in Fig. 5.12, for example, range from $6.5 \times 10^{7}$ to $1.2 \times 10^{8}$. These are perfectly reasonable SM-SERS enhancement factors, comparable to those found in dyes for similar experimental conditions [19]. SM-SERS enhancement factors close to $\sim 10^{10}-10^{11}$ (which are the maximum known to be compatible with electromagnetic theory) are not seen for $\mathrm{C}_{60}$, but we believe it is a real effect which is either related to the photostability of the molecule or its inability to find the highest-enhancement hot-spots; based on its much less favorable interaction with metallic colloids (compared to dyes) or its larger size. Similar enhancement factors were also found for singlemolecule $\mathrm{C}_{60}$ events in the bi-analyte experiments with BPE. Overall, the range of enhancement factors, plus the spectral characteristics, and the frequency spread of the signals, all confirm and reinforce their identification as SM-SERS events of $\mathrm{C}_{60}$.

\subsection{Conclusion}

Besides the demonstration of single molecule detection for the iconic molecule which is $\mathrm{C}_{60}$, we believe the results here highlight one more time the universal character of SM-SERS as a technique, which has already been demonstrated for extreme opposites in the range of cross sections (spanning from resonant dyes [19] to non-resonant and biologically relevant molecules [25]). In addition, our results show, as seen in Chapter 4 
the beauty and simplicity of single molecule spectra to reveal fundamental aspects of spectroscopy of molecules that would not be accessible otherwise. An example of the latter is the observation of isotopic-induced homogeneous broadening [128] in single molecule spectra. The full theoretical explanation of the isotopic-induced broadening goes beyond the harmonic approximation and is not available at present. SM-SERS provides then the experimental motivation to explain phenomena like this, that would remain hidden by ensemble averages in less sensitive types of spectroscopies. 


\section{Chapter 6}

\section{Photostability of dyes under SERS conditions}

This chapter presents the experiments investigating the photobleaching dynamics of Nile blue adsorbed onto regular arrays of gold nanoparticles under typical SERS conditions. Over time, we expect that both the Raman and fluorescence intensities will decrease as the molecules are photobleached; by measuring the time evolution of the SERS intensity we hope to gain insight on how the photobleaching rate is modified by the metallic substrate. Furthermore, as we will see in this chapter, with the presence of the metallic nanostructures, the molecules that experience the highest local field enhancements should, on average, photobleach first. Thus, in theory, measuring the time evolution of the Raman and fluorescence intensities could ultimately give information on the enhancement factor distribution.

\subsection{Background and motivation}

Photobleaching is a well documented subject in dye spectroscopy especially because of its relevance when one deals with fluorescent dyes as probes. Fluorophores are typically used to stain tissues, cells or materials in a variety of analytical methods especially in biochemistry [129] but are also used in Organic Light Emitting Diodes (OLED), solar pan- 
els, etc. Most dyes, under relatively strong resonant excitation tend to photobleach, i.e. they undergo irreversible chemical alteration after some time and hence stop fluorescing (or scattering Raman photons). As many experiments involve the monitoring of a large number of molecules at a time, this results in the decrease of the fluorescence/Raman intensity over time as can be seen in Fig. 6.1 in the case of Nile blue in SERS conditions. The effects of photobleaching of dyes are not always unwelcome; they are indeed at the core of the Fluorescence Recovery After Photobleaching (FRAP) and related techniques which are capable of quantifying the two dimensional lateral diffusion of molecular thin films containing fluorescent dyes by monitoring the extinction of the fluorescence signal due to bleaching and its recovery when still fluorescing probes diffuse through the sample and replace the bleached ones [130].

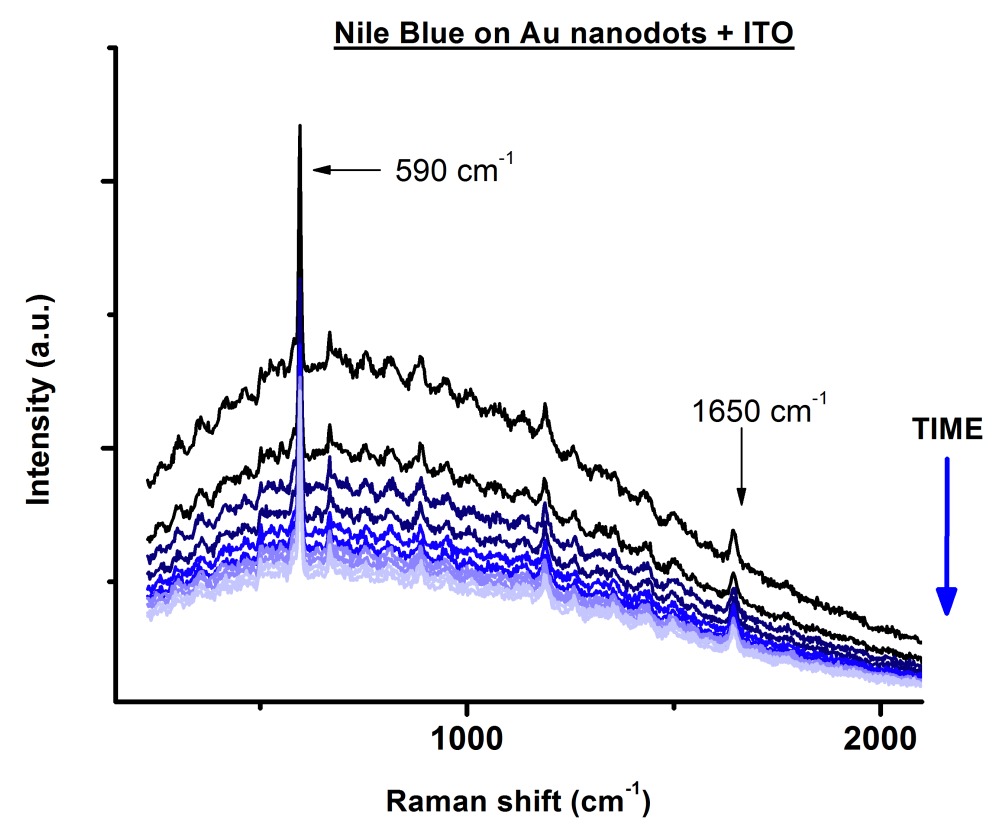

Figure 6.1: Example of the decrease of the SERS signal of Nile blue over time under continuous illumination by the laser. There is a fast initial decay, then the decrease tapers off. Eventually (not shown), the signal disappears.

The exact mechanisms which govern photobleaching are still not well 
understood in all their detail. There is however a conventional explanation for photobleaching which involves inter-system crossing from the first excited singlet state $\left(S_{1}\right)$ to the triplet state $\left(T_{1}\right)$ of the dye which has a relatively long lifetime. From there on, the dye can bleach through interaction with another dye in its triplet state or with an oxygen molecule for example[131, 132]. When one ignores saturation effects, the photobleaching rate increases linearly with the incident power and, at a given power, the observed decay in intensity of the signal (when monitoring a population of equivalent molecules) is exponential with a single photobleaching decay rate.

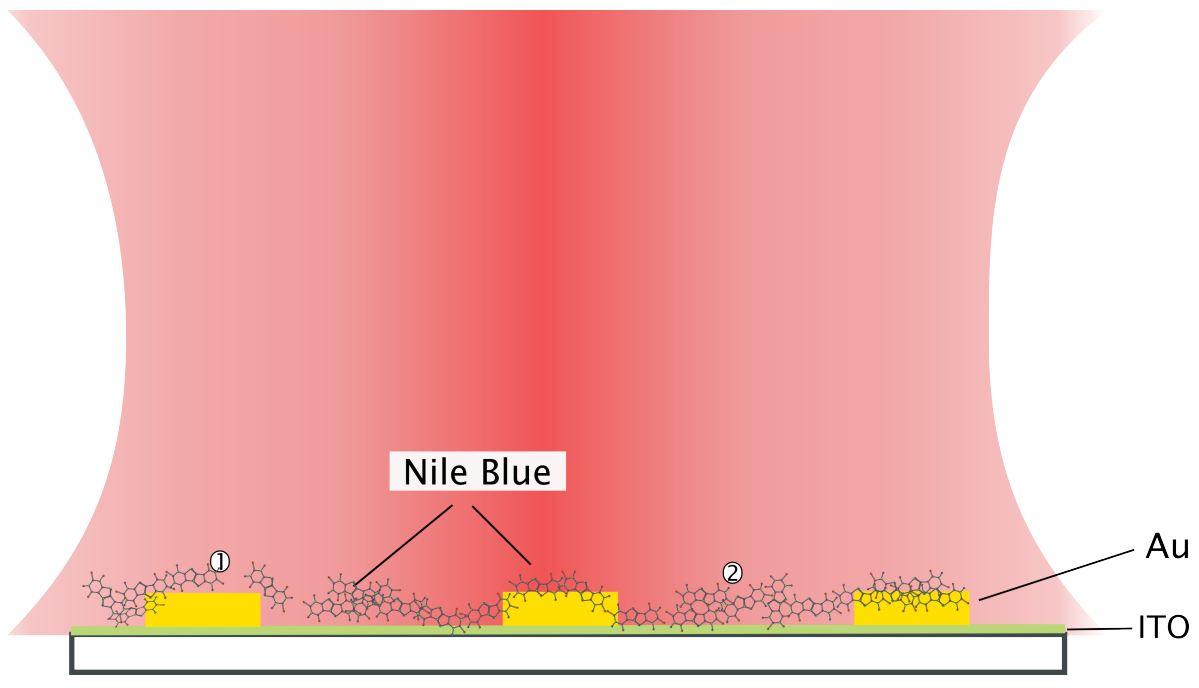

Figure 6.2: Schematics of the system studied in this chapter. A molecule close to the gold surface at (1) will experience a different decay rate than a molecule far from the metal and close to the ITO at (2).

It is then naturally interesting to investigate which changes in the photobleaching effects and rates are brought by the vicinity of a metallic surface as it is relevant to the two growing fields that are SERS and Surface Enhanced Fluorescence (SEF). SEF is the modification (enhancement or quenching) of the fluorescence intensities and lifetimes for molecules adsorbed on or close to (by a few nm) a metal surface [1]. A few studies have been dedicated to this problem; metal-modified non-radiative decay rates [27], spectral modifications in SEF [133], influence of photostability in determining enhancement factors [29, 134, 135], single molecule pho- 
tobleaching [136] and photobleaching under SERS conditions [137, 138].

But a study focusing particularly on the photobleaching dynamics in SERS and SEF has yet to be done. Our hypothesis is that, within the model of photobleaching rates proportional to incident power (through absorption rate and in the case where enhanced non-radiative electromagnetic decay is predominant), the molecules experiencing the highest local field enhancement through the excitation of localized surface plasmons in the metal will have a higher probability of photobleaching than the molecules which are not in the vicinity to a hot spot. The distribution in enhancement felt by different molecules is then expected to be reflected in the intensity dynamics by a distribution of photobleaching rates within the sample. We hope to be able to test this hypothesis by measuring the time evolution of the SERS and SEF intensities of the dye Nile blue adsorbed on gold nanodots arrays which constitute an 'ordered' SERS system, as uniform as possible. Figure 6.2 illustrates schematically the experiment we carry out in this chapter. Nile blue is homogeneously deposited on the gold nanoarrays SERS substrate. The incident laser at $\lambda_{\text {exc }}=633 \mathrm{~nm}$ excites Nile blue resonantly as well as the localized surface plasmons (LSP) of the arrays which have been chosen for their LSP resonance to be in this spectral region. The size of the illuminated area (which also produces the SERS signal) is $\approx 2 \mu \mathrm{m}$ (in red on Fig. 6.2) and is ten times larger than the typical diameter of a nanodot; hence the collected signal will arise from molecules at a variety of distances from the electromagnetic hot spots of the substrate, consequently experiencing a variety of enhancement factors.

\subsection{Photobleaching rate and modified pho- tobleaching rate}

\subsubsection{A simple model for photobleaching}

At low or moderate power, the conventional explanation for photobleaching is via the inter-system crossing phenomenon, i.e. a transition between the singlet and triplet states [131]. Let us consider a simple two-singlet 


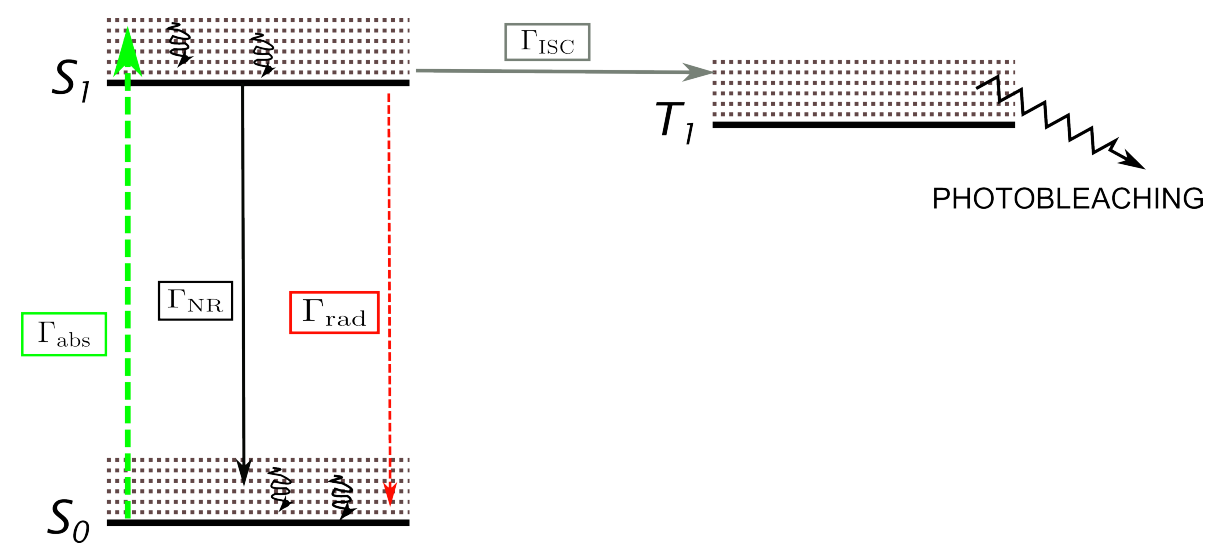

Figure 6.3: Jablonski diagram representing the photobleaching process in a simplified model. Possible relaxation of the electron from $T_{1}$ to the ground state $S_{0}$ is ignored for simplicity.

states + one triplet state system representing the electronic states of the molecule as shown on Fig. 6.3.

Let us define the following rates, expressed in $\mathrm{s}^{-1}$ :

- $\Gamma_{\text {abs }}^{0}$ : the absorption rate; it is the number of photons absorbed by the molecule per unit time. $\Gamma_{\text {abs }}^{0}$ is proportional to the incident power density and to the absorption cross-section of the molecule. Thus if $S_{\mathrm{inc}}$ is the excitation density (photon $\mathrm{s}^{-1} \mathrm{~m}^{-2}$ ), $\Gamma_{\mathrm{abs}}^{0}=S_{\mathrm{inc}} \sigma_{\mathrm{abs}}$.

- $\Gamma_{\text {rad }}^{0}$ : the radiative decay rate; it corresponds to the probability per unit time of emission of a photon through spontaneous emission by the molecule in its electronic excited state $S_{1}$.

- $\Gamma_{\mathrm{NR}}^{0}$ : the non-radiative decay rate; the probability per unit time for the molecule to undergo a non-radiative transition from $S_{1}$ to $S_{0}$. Spontaneous emission is not the only relaxation path for an electron from $S_{1}$ to $S_{0}$, there are non-radiative transitions from $S_{1}$ to $S_{0}$ and they may have several origins [1]. Their overall effect is described by $\Gamma_{\mathrm{NR}}^{0}$. This is an intrinsic property of the molecule and its solvent and there is no photon emission involved.

- $\Gamma_{\mathrm{Tot}}^{0}$ : the total decay rate. It represents the true decay rate of the excited state $S_{1} \cdot \Gamma_{\text {Tot }}^{0}=\Gamma_{\text {rad }}^{0}+\Gamma_{\mathrm{NR}}^{0}$. 
- $\Gamma_{\text {ISC }}^{0}$ : probability of inter-system crossing per unit time. Typically, $\Gamma_{\text {ISC }}^{0} \ll \Gamma_{\text {rad }}^{0}$.

Let us define furthermore the fluorescence quantum yield $Q_{0}$ :

$$
Q_{0}=\frac{\Gamma_{\mathrm{rad}}^{0}}{\Gamma_{\mathrm{Tot}}^{0}}=\frac{\Gamma_{\mathrm{rad}}^{0}}{\Gamma_{\mathrm{rad}}^{0}+\Gamma_{\mathrm{NR}}^{0}}
$$

The quantum yield is the proportion of excited electrons that decays radiatively to the ground state. The quantum yield of Nile blue is $Q_{0}=$ 0.27 in ethanol and 0.04 in water [7].

When the molecule is excited in $S_{1}$, the probability of photobleaching through inter-system crossing is then:

$$
\begin{aligned}
p_{\mathrm{B}}^{0} & =\frac{\Gamma_{\mathrm{ISC}}^{0}}{\Gamma_{\mathrm{Tot}}^{0}} \\
& =\frac{\Gamma_{\mathrm{ISC}}^{0}}{\Gamma_{\mathrm{rad}}^{0} Q_{0}^{-1}}
\end{aligned}
$$

When we ignore saturation effects, the number of excitation/relaxation cycles per unit time is given by $\Gamma_{\text {abs }}^{0}$. For each of these cycles, there is a probability $p_{\mathrm{B}}^{0}$ that the molecule crosses to the triplet state and photobleaches. The total number of molecules $N$ then decreases exponentially with a photobleaching rate $\Gamma_{\mathrm{PB}}^{0}$ :

$$
N(t)=N_{0} e^{-\Gamma_{\mathrm{PB}}^{0} t} \quad \text { with } \quad \Gamma_{\mathrm{PB}}^{0}=p_{\mathrm{B}}^{0} \Gamma_{\text {abs }}^{0}=\alpha S_{\mathrm{inc}}
$$

$\alpha$ being some proportionality constant.

Hence the photobleaching rate increases linearly with power and we can give a phenomenological formula for the time evolution of the Raman intensity:

$$
I\left(t, S_{\mathrm{inc}}\right)=I_{0} e^{-\Gamma_{\mathrm{PB}}^{0} t}=I_{0} e^{-\alpha S_{\mathrm{inc}} t}
$$

Equation 6.3 is valid when the excitation is uniform and all the molecules experience the same power density; in practice this is not true for typical laser beams and their non uniformity will result in a different $\Gamma_{\mathrm{PB}}$ for 
different molecules.

\subsubsection{Modified photobleaching rate in the presence of the metallic surface}

Under typical SERS conditions, the polarizable molecule radiates, not in an infinite dielectric medium, but in close proximity to a metallic surface. Absorption and spontaneous emission are modified. In order to know how it affects the photobleaching rate defined above, let us take a closer look at the several enhancement factors we can define for the local field intensity and the emitted, radiated and total powers experienced by the molecule when in the presence of the metal with respect to free-space (molecule in an infinite dielectric medium) [1].

- The local electric field $\vec{E}_{\text {loc }}$ at the molecule's position near to the surface is modified and different from the incident field $\vec{E}_{\text {inc }}$. The local field intensity enhancement factor is $M_{\mathrm{loc}}=\frac{\left|\vec{E}_{\mathrm{loc}}\right|^{2}}{\left|\vec{E}_{\mathrm{inc}}\right|^{2}}$. Absorption is subject to the local field enhancement and then $\Gamma_{\mathrm{abs}}=M_{\mathrm{loc}} \Gamma_{\mathrm{abs}}^{0}$ for a molecule randomly oriented on the surface.

- The radiated power emitted in all directions by spontaneous emission is modified by a factor $M_{\text {rad }}$. The modified radiative decay rate is then $\Gamma_{\text {rad }}=M_{\text {rad }} \Gamma_{\text {rad }}^{0}$. Because of the optical reciprocity theorem, we can assume that $M_{\mathrm{rad}} \approx M_{\mathrm{loc}}$.

- The enhancement in the total electromagnetic energy extracted from the dipole (emitting molecule) is characterized by the total electromagnetic enhancement factor $M_{\text {Tot }}$. It encompasses two situations from the point of view of modified spontaneous emission; part of the electromagnetic energy will be emitted in the far field (radiative) and part of this energy will be directly absorbed by the metallic substrate, hence undetectable (non-radiative, even if mediated by photon emission). Hence, the modified total decay rate for the excited state is now: $\Gamma_{\mathrm{Tot}}=M_{\mathrm{Tot}} \Gamma_{\mathrm{rad}}^{0}+\Gamma_{\mathrm{NR}}^{0}$, where the intrinsic (non-electromagnetic, non-modified) non-radiative decay rate of the molecule $\Gamma_{\mathrm{NR}}^{0}$ is added. 
With these definitions in mind, we can define the SERS and SEF enhancement factors with their dependence on the excitation $\left(\lambda_{L}\right)$ and emission $\left(\lambda_{S}\right)$ wavelengths [27]:

$$
M_{\mathrm{SERS}}=M_{\mathrm{loc}}\left(\lambda_{L}\right) M_{\mathrm{rad}}\left(\lambda_{S}\right) \quad, \quad M_{\mathrm{SEF}}=\frac{M_{\mathrm{loc}}\left(\lambda_{L}\right) M_{\mathrm{rad}}\left(\lambda_{S}\right)}{Q_{0} M_{\mathrm{Tot}}}
$$

Within the $|E|^{4}$-approximation of the enhancement factor [11], we can consider that:

$$
M_{\mathrm{SERS}}=M_{\mathrm{loc}}^{2}
$$

The study of the ratio

$$
R_{\mathrm{EF}}=\frac{M_{\mathrm{SERS}}}{M_{\mathrm{SEF}}}=Q_{0} M_{\mathrm{Tot}}
$$

(through the ratio of SERS and SEF intensities), can give experimental access to the total electromagnetic enhancement factor $M_{\text {Tot }}$ [27].

We suppose that $\Gamma_{\text {ISC }}$ is not changed by the presence of the metallic surface as no emission/absorption of a photon is involved. Let us call $p_{\mathrm{B}}$ and $\Gamma_{\mathrm{PB}}$ the modified photobleaching probability for a molecule in $S_{1}$ and the modified photobleaching rate respectively.

$$
\begin{aligned}
p_{\mathrm{B}} & =\frac{\Gamma_{\mathrm{ISC}}^{0}}{\Gamma_{\mathrm{Tot}}} \\
& =\frac{\Gamma_{\mathrm{Tot}}^{0}}{\Gamma_{\mathrm{Tot}}} p_{\mathrm{B}}^{0} \\
& =\frac{p_{\mathrm{B}}^{0}}{Q_{0} M_{\mathrm{tot}}+1-Q_{0}}
\end{aligned}
$$

because we can write $\Gamma_{\text {Tot }}=\left(M_{\text {Tot }}+Q_{0}^{-1}-1\right) \Gamma_{\text {rad }}^{0}$. Hence, once the molecule is in the excited state $S_{1}$, the probability of photobleaching is mainly governed and often reduced by an amount $\approx Q_{0} M_{\text {Tot }}$ (which can be large under SERS conditions).

However the modified photobleaching rate also contains contributions from the enhanced absorption, i.e. many more excitation/relaxation cy- 
cles per unit time:

$$
\begin{aligned}
\Gamma_{\mathrm{PB}} & =p_{\mathrm{B}} \Gamma_{\mathrm{abs}} \\
& =p_{\mathrm{B}} M_{\mathrm{loc}} \Gamma_{\mathrm{abs}}^{0}
\end{aligned}
$$

The modified photobleaching rate is still proportional to the incident power through the dependence in $\Gamma_{\text {abs }}$. The modification of the photobleaching rate then takes the form:

$$
\begin{aligned}
\frac{\Gamma_{\mathrm{PB}}}{\Gamma_{\mathrm{PB}}^{0}} & =M_{\mathrm{loc}} \frac{p_{\mathrm{B}}}{p_{\mathrm{B}}^{0}} \\
& =\frac{M_{\mathrm{loc}}}{Q_{0} M_{\mathrm{Tot}}+1-Q_{0}}
\end{aligned}
$$

For most dyes with a non-small $Q_{0}$, the modified total electromagnetic decay rate $M_{\mathrm{Tot}} \Gamma_{\text {rad }}^{0}$ will be much larger than the intrinsic non-radiative decay rate $\Gamma_{\mathrm{NR}}^{0}=\left(Q_{0}^{-1}-1\right) \Gamma_{\mathrm{rad}}^{0}$ provided that $M_{\mathrm{loc}} \gg Q_{0}$. For Nile blue in ethanol, $Q_{0}^{-1} \approx 4$ and single molecule SERS gains of $10^{6}$ are easily achieved which means $M_{\mathrm{loc}} \approx 10^{3} \gg 4$. Hence, Eq. 6.10 becomes:

$$
\frac{\Gamma_{\mathrm{PB}}}{\Gamma_{\mathrm{PB}}^{0}} \approx \frac{M_{\mathrm{loc}}}{Q_{0} M_{\mathrm{Tot}}}
$$

Two important remarks can be made here:

- If $M_{\text {tot }}$ is dominated by radiative emission, then $M_{\text {Tot }} \approx M_{\text {rad }} \approx$ $M_{\mathrm{loc}}$ and the modified photobleaching rate does not depend on $M_{\mathrm{loc}}$ any more; different molecules experiencing different local enhancement will exhibit the same photobleaching dynamics, within the approximations of this model.

- If $M_{\text {Tot }}$ is dominated by non-radiative emission of photons to the metal (which is the case for Nile blue), $M_{\text {loc }} \ll M_{\text {Tot }}$ and $\Gamma_{\mathrm{PB}}$ can be greatly reduced compared to the free-space case. Moreover, $\Gamma_{\mathrm{PB}}$ is then proportional to $M_{\text {loc }}$, which means that the photobleaching rate will be larger for molecules subject to larger enhancement.

Similarly to Eq. 6.4, we can write a phenomenological formula for the time-evolution of the SERS intensity for one molecule close to a metallic 
surface:

$$
I_{\mathrm{SM}}\left(t, S_{\mathrm{inc}}, M_{\mathrm{loc}}\right)=I_{\mathrm{SM}}^{t=0} e^{-\Gamma_{\mathrm{PB}} t} \propto S_{\mathrm{inc}} M_{\mathrm{loc}}^{2} e^{-\beta M_{\mathrm{loc}} S_{\mathrm{inc}} t}
$$

$\beta$ being some proportionality constant. Therefore, when many molecules with different enhancements contribute to the signal, the time evolution of the total SERS intensity will show a non-exponential decay:

$$
\begin{aligned}
I_{\mathrm{Tot}}\left(t, S_{\mathrm{inc}}\right) & =I_{\mathrm{Tot}}^{0} \int_{\Gamma_{\mathrm{PB}}} f\left(\Gamma_{\mathrm{PB}}\right) e^{-\Gamma_{\mathrm{PB}} t} \mathrm{~d} \Gamma_{\mathrm{PB}} \\
& \propto S_{\mathrm{inc}} \int_{0}^{\infty} f\left(M_{\mathrm{loc}}\right) M_{\mathrm{loc}}^{2} e^{-\beta M_{\mathrm{loc}} S_{\mathrm{inc}} t} \mathrm{~d} M_{\mathrm{loc}}
\end{aligned}
$$

with $f\left(M_{\text {loc }}\right) d M_{\text {loc }}$ the probability distribution of enhancement factors on the surface [13].

The aims of the study presented in this chapter are to:

- Design the experimental methods: data acquisitions and background measurements.

- Check the model described in this section against the experimental data.

- Determine whether the decay of the signal intensity reveals information on the form of $f\left(M_{\mathrm{loc}}\right)$.

\subsection{Experimental methods}

This section presents the SERS substrate used for the study, the sample preparation and the necessary steps which had to be taken in order to analyze the data.

\subsubsection{The SERS substrate: gold nanoparticle arrays}

To carry out this study, a uniform and reproducible SERS substrate was desirable. We used gold nanoparticle arrays which have been fabricated by electron beam lithography at the ITODYS laboratory (In- 
terfaces, Traitements, Organisation et Dynamique des Surfaces) by Dr Johan Grand in Université Paris-Diderot (France).

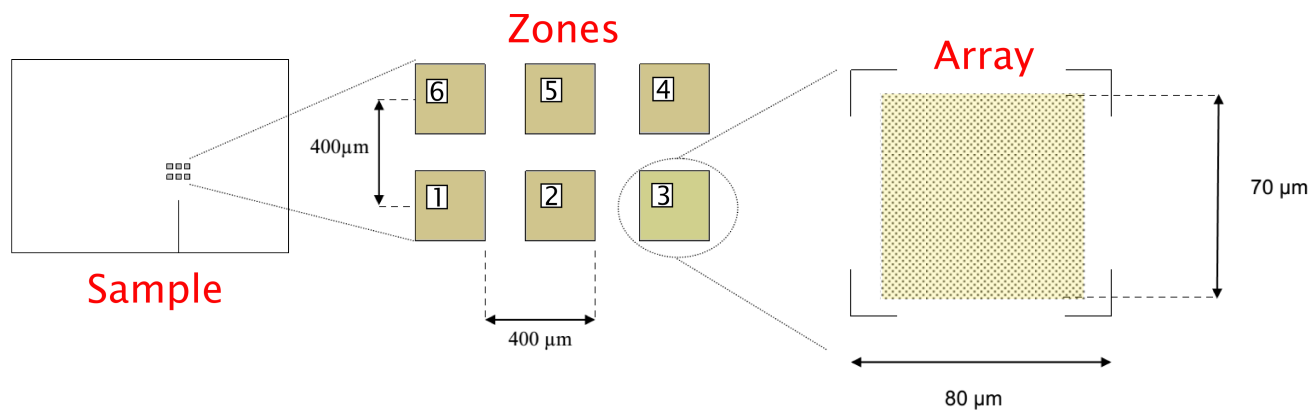

Figure 6.4: $50 \mathrm{~nm}$ gold nanodots on ITO arrays fabricated by e-beam lithography. Each zone corresponds to a particular pitch.

The substrates consist of gold nanodots $50 \mathrm{~nm}$ thick on Indium Tin Oxide (ITO) arranged in arrays of variable pitch: ITO is chosen for

\begin{tabular}{|l|c|c|}
\hline & Diameter $[\mathrm{nm}]$ & Pitch $[\mathrm{nm}]$ \\
\hline Zone 1 & 150 & 300 \\
\hline Zone 2 & 130 & 280 \\
\hline Zone 3 & 110 & 260 \\
\hline Zone 4 & 90 & 240 \\
\hline Zone 5 & 70 & 220 \\
\hline Zone 6 & 50 & 200 \\
\hline
\end{tabular}

Table 6.1: Theoretical values of the particle diameter and array pitch for each zone.

its optical transparency and electrical conductivity required for electron beam exposure and Scanning Electron Microscopy (SEM) imaging. The fundamental mechanisms of SERS on ordered arrays of gold nanoparticles have been studied in detail in [139, 140], especially how varying the size, shape and spacing of such particles influences their optical near field properties (SERS enhancement) and far field properties (extinction spectra). According to the Weitz's phenomenological relationship [141] and the work published in [139] for arrays of gold nanoparticles, the desired maximum of the localized surface plasmon (LSP) resonance wavelength $\lambda_{\max }$ should be related to the excitation wavelength $\lambda_{\text {exc }}$ and Raman 
scattering wavelength $\lambda_{\mathrm{RS}}$ for this excitation by (assuming there is great regularity in the particles size and shape) :

$$
\lambda_{\max }=\frac{\lambda_{\mathrm{exc}}+\lambda_{\mathrm{RS}}}{2}
$$

Thus, when exciting Nile blue resonantly at $\lambda_{\max }=633 \mathrm{~nm}$ and focusing on the Raman mode at $590 \mathrm{~cm}^{-1}\left(\lambda_{\mathrm{RS}} \approx 658 \mathrm{~nm}\right)$, the LSP resonance of the gold arrays should ideally be located at or near $\lambda_{\max } \approx 645.5 \mathrm{~nm}$. This is why, out of the 6 zones available on the substrate, zones 1 and 2 are particularly suited for SERS on the $590 \mathrm{~cm}^{-1}$ mode of Nile Blue at $633 \mathrm{~nm}$ as can be seen in Fig. 6.5 with LSP resonances for zone 1 at $680 \mathrm{~nm}$ and zone 2 at $650 \mathrm{~nm}$.

\subsubsection{Sample preparation}

The gold nanonarrays were cleaned in ethanol then dipped into a solution of Nile blue A $1.2 \mu \mathrm{M}$ then rinsed (by dipping) into ultrapure water and dried under $\mathrm{N}_{2}$ flow.

\subsubsection{Acquiring Raman and SERS spectra with the $1 \mathrm{MHz}$ standard mode of the CCD detector}

\section{The CCD detector}

The experiments of this chapter were made using the CCD (chargecoupled device) detector in the $1 \mathrm{MHz}$ standard mode (whereas the measurements of the previous chapters were all recorded with the CCD detector in the $20 \mathrm{kHz}$ mode). Our CCD detector is a Symphony CCD detection system $\mathrm{LN}_{2}$ series from Horiba-Jobin Yvon. The data conversion speed can be set to $20 \mathrm{kHz}$ or $1 \mathrm{MHz}$ via the software Labspec. In order to access the dynamics of the SERS signal of Nile blue, we need to collect data as a function of time and the speed and accuracy of the data collection is hence crucial.

The spectrometer is set to a specific grating position by the software (Labspec); when the experiment is running, the CCD collects data only 
ZONE 1

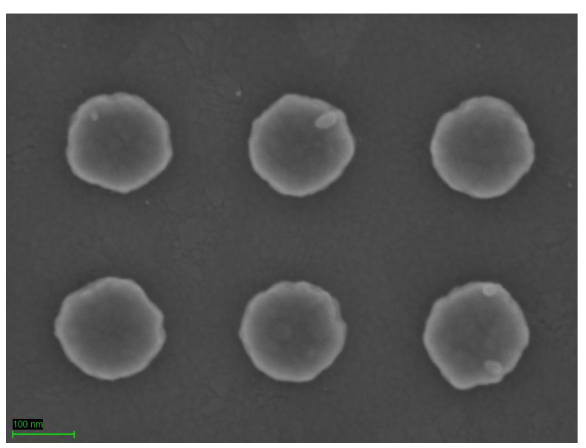

(a) SEM images of zones 1 and 2. The scale bar is $100 \mathrm{~nm}$ on the left and $200 \mathrm{~nm}$ on the right.

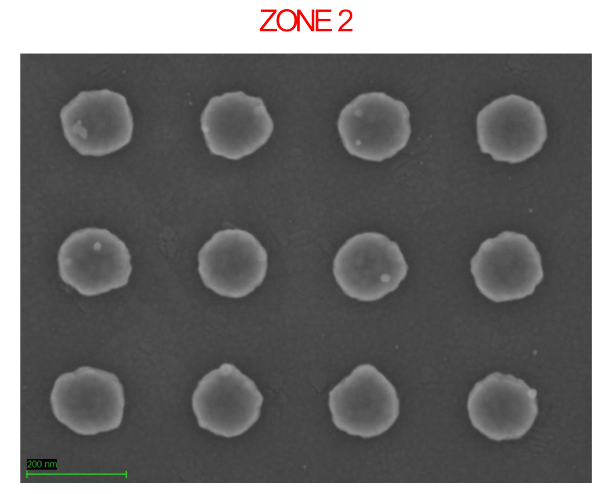

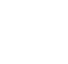


from the range of wavelengths that reach the detector for a length of time IT (integration time). Each column of pixels (or binned columns) of the CCD is then mapped to a single wavelength (the step between these wavelengths or resolution is set by how dispersive the grating is). The resulting data is a spectrum with a signal intensity value for each column of pixels. In a time dependent experiment, the experiment is repeated throughout a specific time period with a time step between acquisitions $\delta t^{\prime}$. The data is stored as a list of spectra as a function of time. When the chosen step between 2 acquisition is small enough (typically $\leq 0.1 \mathrm{~s}$ ), the CCD runs in the so-called kinetic mode; the shutter in front of the CCD opens at the beginning of the time-dependent experiment and closes once all the spectra have been recorded.

When carrying out such a time-dependent experiment, one has to set up some parameters via the software:

* The integration time $\mathrm{IT}^{\prime}$, which is the amount of time the CCD is exposed to light AND acquires data. The knowledge of the accurate integration time corresponding to a spectrum enables one to make meaningful comparisons between spectra.

* The time interval $\Delta t^{\prime}$ between the start of one accumulation to the start of the next accumulation. $\Delta t^{\prime}$ has to be larger than $\mathrm{IT}^{\prime}+t_{\text {read }}$ where $t_{\text {read }}$ is the read-out time of the CCD. When $\Delta t^{\prime}-\mathrm{IT}^{\prime} \leq 0.1 \mathrm{~s}$ the CCD runs automatically in kinetic mode.

The data is saved via Labspec in a text file containing a series of spectra with the corresponding times $t^{\prime}$ at which their recording has begun. We expect that the time evolution of the SERS/fluorescence signal of Nile blue on the gold nanoarrays substrates will involve many decay rates (see Sec. 6.2.2) and the characteristic decay times experienced by each molecule likely span several orders of magnitude (from ns to s). As our set-up (in $1 \mathrm{MHz}$ mode) does not allow for integration times smaller than $0.027 \mathrm{~s}$, if we want to access the dynamics at full incident power $P$ during the first $0.03 \mathrm{~s}$, then, within the model presented in Sec. 6.2 it should be equivalent to study the dynamics of the system at 
$P / 10$ for the first $0.3 \mathrm{~s}$, etc. To be able to compare spectra at different powers and integration times between each other we need to know:

- What the real integration times are. Are the values saved in the data files reliable?

- What the actual incident power is when optical density filters are used.

\section{Integration times and CCD latency in the $1 \mathrm{MHz}$ mode Standard}

\section{: Labspec versus Reality}

The Analog to Digital Conversion (ADC) speed can be set by choosing the rate at which the data is read off the CCD detector. For maximum signal-to-noise ratio, the ADC speed was set to $20 \mathrm{kHz}$ for the experiments presented in the previous chapters; however, for the study of the present chapter, the use of the maximum frame rate $(1 \mathrm{MHz})$ is desirable as it reduces the latency between the acquisition of consecutive spectra.

When carrying out time series of measurements with Labspec in kinetic mode, several issues appear: firstly the measurement does not start exactly when the shutter opens (the acquisition starts after a certain $t_{\text {in }}$ after the shutters opens). Then, the Labspec times for each spectrum are incorrect (there is a dilatation factor $r$ between the real times $t$ and the times given by Labspec in the data file $t^{\prime}$. Finally, the Labspec integration times are incorrect and governed by the time interval $\Delta t^{\prime}$; in short, for the same specified integration times $\mathrm{IT}^{\prime}$ for each spectrum but two different time intervals $\Delta t^{\prime}$, two time-series experiments will in fact have two different real integration times IT. Labspec times are not reliable.

A quick experiment consisting of recording Raman time-series on the $520 \mathrm{~cm}^{-1}$ mode of silicon for different integration times and different time intervals between spectra and comparing the intensity of the peak each time yielded the following result: in the $1 \mathrm{MHz}$ Standard kinetic mode of the CCD, Labspec ignores the user specified step size between spectra $\left(\Delta t^{\prime}\right)$ and the real integration time IT for each spectrum will be:

$$
I T=\Delta t=I T^{\prime}+0.016 \mathrm{~s}
$$


where $\Delta t$ is the real interval time between two consecutive spectra in a time-series, $I T^{\prime}$ is the integration time set by the user via the software and $t_{\text {read }}=0.016 \mathrm{~s}$ in the high frame rate $1 \mathrm{MHz}$ mode. Eq. 6.15 enables us then to set up the time-series properly and accordingly scale the spectra by integration times when analyzing the data.

Moreover, the quick study above also enabled us to get $t_{\text {in }}$ for each configuration. It takes around a second for the data acquisition to start when the shutter was opened. Thus we started each time-series for the NB dynamics study with the laser off and turned it on after about 2 seconds.

\subsubsection{Optical density filters comparison}

Beside the real integration times, the knowledge of the incident power on the sample is important, especially when one wants to compare spectra between each others. In order to modulate the incident power on the sample, optical density (OD) filters are placed on the optical path at the exit from the laser. Table 6.2 shows the effect of the available OD filters on our system.

\begin{tabular}{|c|c|c|c|}
\hline Optical Density & Intensity $[$ counts $/ \mathrm{s}]$ & $-\log \left(I / I_{\text {ref }}\right)$ & $I / I_{\text {ref }}$ \\
\hline D0 $^{a}$ & $210425^{b}$ & 0 & 1 \\
\hline D0.3 & 119468 & 0.25 & 0.57 \\
\hline D0.6 & 58133 & 0.56 & 0.28 \\
\hline D1 & 20754 & 1.0 & 0.099 \\
\hline D2 & 1999 & 2.02 & 0.0095 \\
\hline D3 & 335 & 2.8 & 0.0016 \\
\hline
\end{tabular}

Table 6.2: Optical density filter effect on the incident power at $633 \mathrm{~nm}$.

\footnotetext{
${ }^{a}$ Standard notation of the filters: D followed by the $\log _{10}$ of the nominal factor by which the power is decreased.

${ }^{b}$ This value was used as $I_{\text {ref }}$.

These values were obtained with the He-Ne excitation $\lambda_{\text {inc }}=633 \mathrm{~nm}$, the $\times 50$ LWD objective on a silicon wafer. A fit of the $520 \mathrm{~cm}^{-1}$ peak of silicon to a Lorentzian profile was done for each power to get the intensities. Experiments were done at D3 to D0 with different integration
} 
times (given in Table 6.3).

Table 6.3 summarizes the scaling factors regarding integration times and incident powers on the sample which will be use to analyze the data. When the spectra are rescaled (time and intensity) they will be as to be equivalent to D0 incident power with $1 \mathrm{~s}$ acquisition time.

\begin{tabular}{|c|c|c|c|}
\hline Power & Real IT $(\mathrm{s})$ & Power factor & Total scaling factor: D0 for 1s \\
\hline D3 & 1.02 & 628.13 & 615.44 \\
\hline D2 & 0.515 & 105.23 & 204.21 \\
\hline D1 & 0.117 & 10.14 & 87.17 \\
\hline D0 & 0.026 & 1 & 38.46 \\
\hline
\end{tabular}

Table 6.3: Power and time scaling factors used for the experiments of this chapter.

\subsubsection{Background corrections}

\section{Notch filter response function}

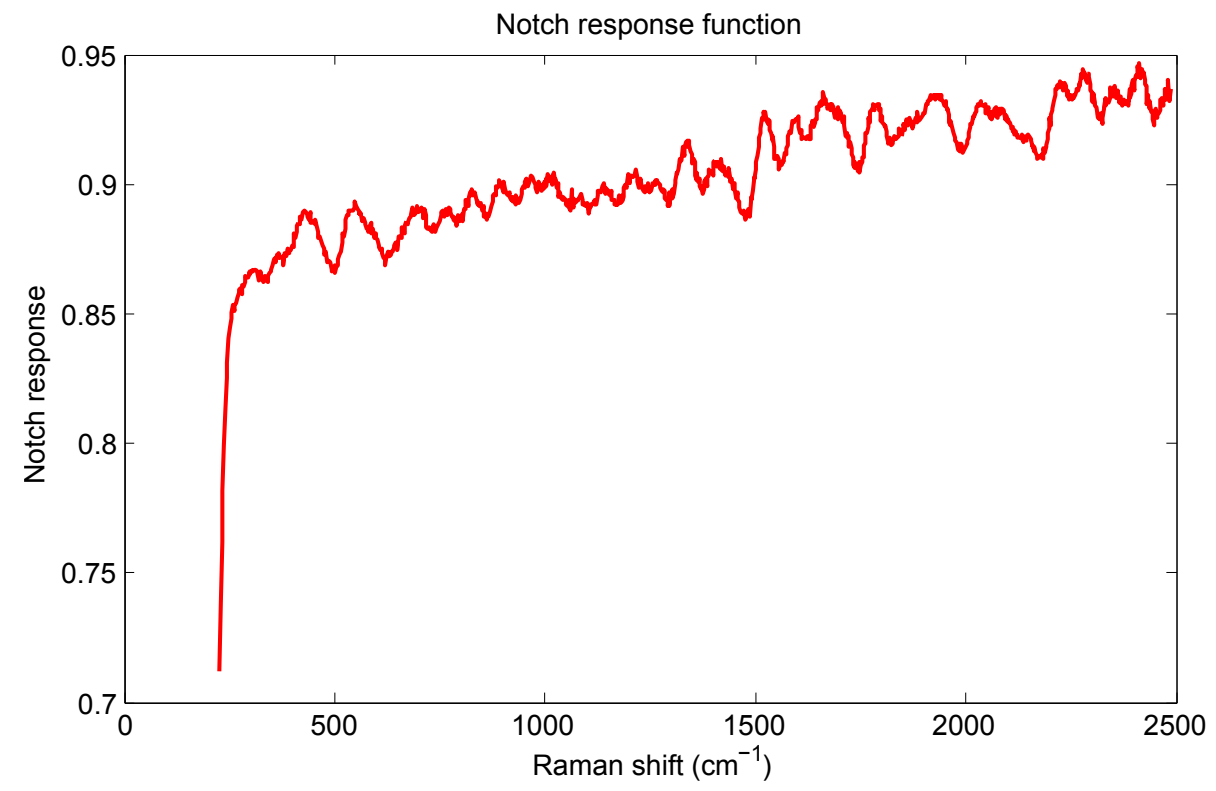

Figure 6.6: Notch response function over the range of wavenumbers of interest. 
We measured the notch filters response function as the ratio:

$$
\text { Notch response function }=\frac{\text { white light spectrum with notch }}{\text { white light spectrum without notch }}
$$

All the data were corrected by the notch response function, the latter being measured on the same day the SERS experiments were done.

\section{$\mathrm{ITO}+\mathrm{Au}$ background}

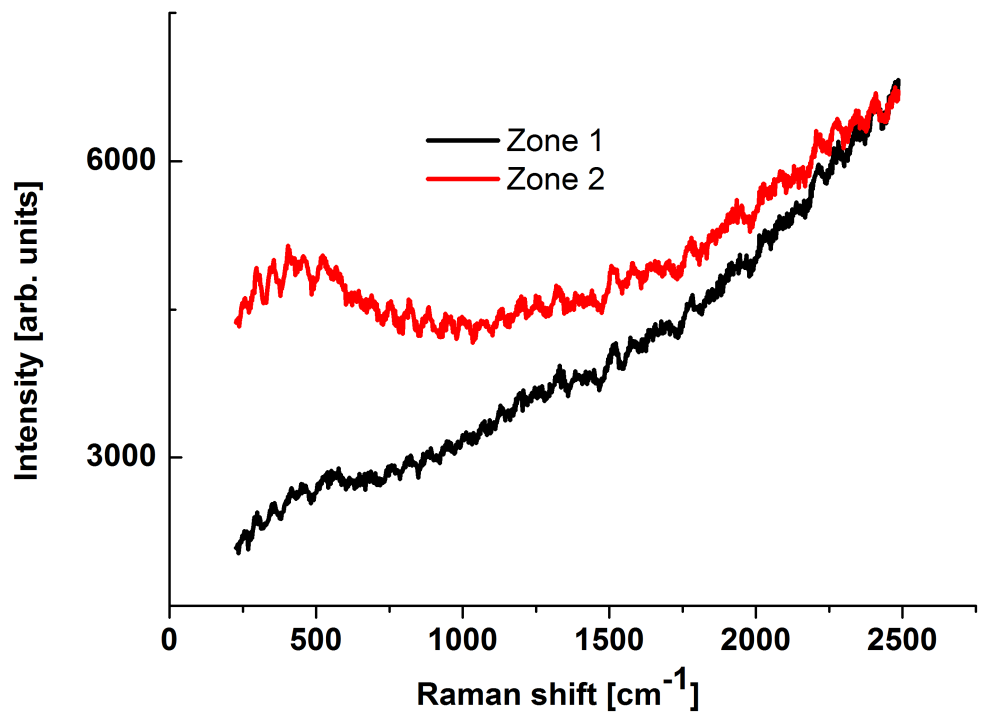

Figure 6.7: Bare zones 1 and 2 background signal. $\lambda_{\text {inc }}=633 \mathrm{~nm}, \mathrm{P}_{\text {inc }}=$ $2.5 \mathrm{~mW}, 1 \mathrm{~s}$ acquisition, averaged over 5 spectra.

We then measured the background created by the bare gold and ITO (before Nile Blue deposition) for zone 1 and zone 2; see Fig. 6.7. These backgrounds affect the total background of the SERS signals of NB, especially at high powers and need to be subtracted from the data.

\subsection{Preliminary experiments and results}

As a large part of the work was dedicated to developing experimental methods, only preliminary studies of photobleaching were carried out 
and they are presented in the following section.

The experiments consist of recording time series of SERS spectra of Nile blue deposited on the gold nanostructures presented in Sec. 6.3.1 at different incident powers. We focus particularly on the time evolution of the $590 \mathrm{~cm}^{-1}$ and $1650 \mathrm{~cm}^{-1}$ modes' average SERS intensities and that of the fluorescence background.

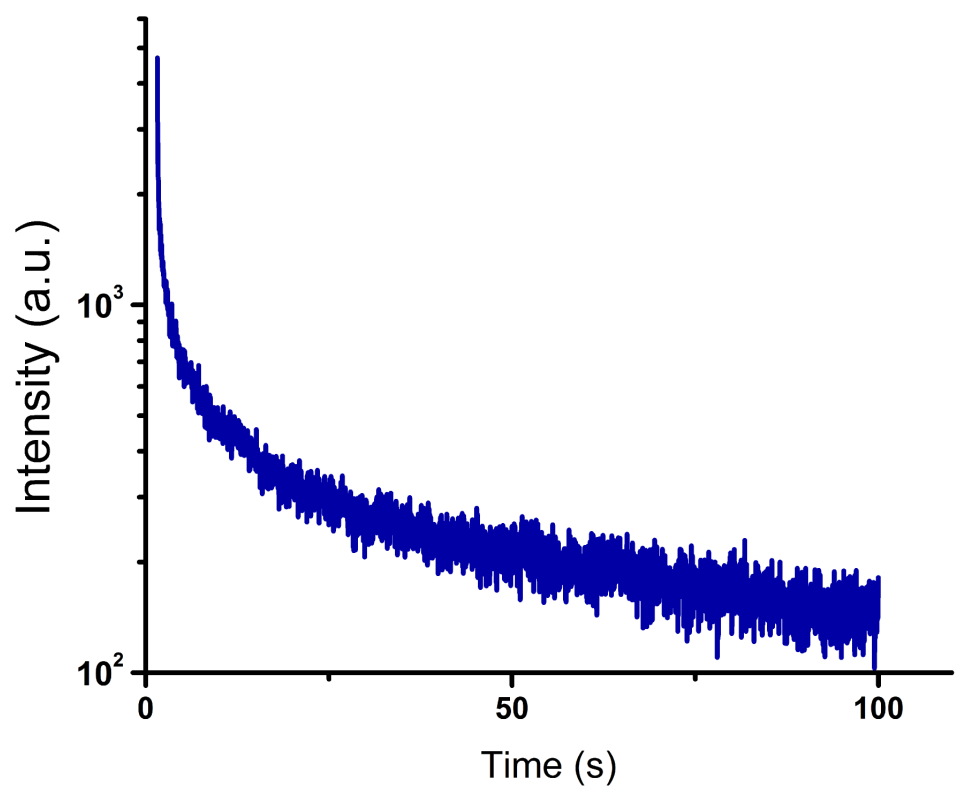

Figure 6.8: Evolution of the SERS intensity of the $590 \mathrm{~cm}^{-1}$ mode of Nile blue at D0. The step between two consecutive spectra was $0.03 \mathrm{~s}$. The decay in intensity is non-exponential.

The excitation wavelength used is the $633 \mathrm{~nm}$ line from the He-Ne laser. For the purpose of these experiments, the Labram spectrometer is equipped with a double notch filter to remove stray light coming from reflections and scattering of the sample and access the true background of the signal. The CCD is run in the $1 \mathrm{MHz}$ Standard mode. We use the lower resolution $300 \mathrm{l} / \mathrm{mm}$ gratings in order to visualize the $590 \mathrm{~cm}^{-1}$ and $1650 \mathrm{~cm}^{-1}$ peaks of NB in the same spectrum. The light is focused on the sample and collected by the $\times 50$ LWD objective (N.A. 0.35 ) which corresponds to an illumination diameter of $\approx 2 \mu \mathrm{m}$ at focus on the sample. 
In this section the incident power on the sample at D0 (no filter) is always $\mathrm{P}_{\mathrm{D} 0}=6.5 \mathrm{~mW}$ and hence $S_{\text {inc }} \approx 1 \mathrm{~mW} \mathrm{\mu m}^{-2}$. The laser was focused on different points of Zone 2 and several time-series of spectra were recorded for powers from D3 $\left(\mathrm{P}_{\mathrm{D} 0} / 1000\right)$ to D0.

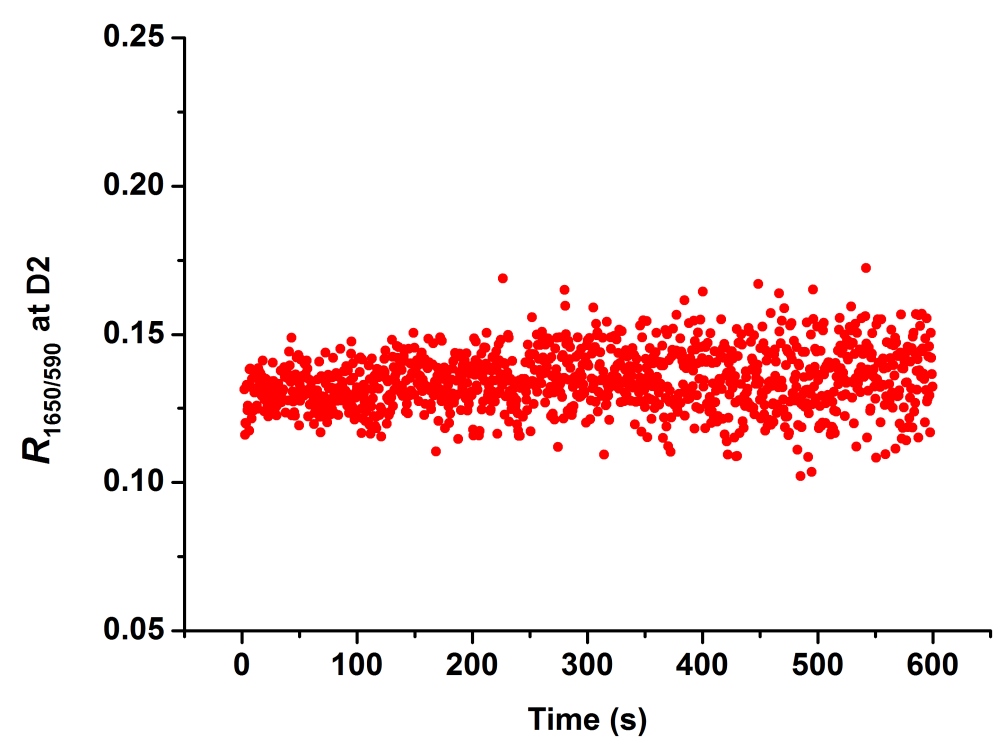

Figure 6.9: Time evolution of the ratio $R_{1650 / 590}$ of SERS intensities measured at D2.

Fig. 6.8 shows the typical evolution of the SERS intensity of the $590 \mathrm{~cm}^{-1}$ mode of Nile blue at maximum incident power. In order to get the values for the SERS intensities, the spectra were corrected for the notch filter response, then a linear fit of all the spectra of the series was performed (see Sec. 3.2) with the average spectrum of the time-series used as reference. These very fast fits yield for each time-series, the SERS intensities of the $590 \mathrm{~cm}^{-1}$ and $1650 \mathrm{~cm}^{-1}$ modes together with the background height (approximated to being linear over the width of the peaks) underneath the peaks. We can can see on Fig. 6.8 that the decay in intensity is strongly non-exponential; it confirms what we expected, i.e. that the global photobleaching dynamics of the molecules consist of many decay rates.

The ratios of the SERS intensities $R_{1650 / 590}=\frac{I_{\text {SERS }}(1650)}{I_{\text {SERS }}(590)}$ were found 
to be constant over time and for the four different powers; this is a sign that the plasmon resonance does not change over time, so the nanodots do not change their shape significantly over laser excitation (see Fig. 6.9), as expected.

In Fig. 6.10 the evolution of the ratios $R_{\mathrm{SERS} / \mathrm{SEF}}=\frac{I_{\mathrm{SERS}}}{I_{\mathrm{SEF}}}$ for the $590 \mathrm{~cm}^{-1}$ and $1650 \mathrm{~cm}^{-1}$ modes for the four powers is plotted. This ratio is proportional to the ratio of enhancement factors $R_{\mathrm{EF}}$ defined in Eq. 6.7:

$$
R_{\mathrm{SERS} / \mathrm{SEF}}=\frac{R_{\mathrm{EF}}}{R_{\text {Raman/Fluo }}}
$$

where $R_{\text {Raman/Fluo is the ratio of Raman over fluorescence intensities }}$ for the bare molecule. Hence, according to Eq.6.7, the evolution of $R_{\text {SERS/SEF }}$ reflects the evolution of $M_{\text {Tot }}$, the total decay rate enhancement. We can see on Fig. 6.10 that the ratios of intensities vary by just a factor of 2-3 for the four considered powers. According to Eq. 6.11, if $M_{\text {Tot }}$ was dominated by radiative emission (i.e. the plasmon resonance mainly affects the radiative processes), then $M_{\text {Tot }}$ would be of the order of $M_{\text {loc }}$; in that case we would see a strong decay of many orders of magnitude of $R_{\text {Raman/Fluo }}$ over time. The evolution of $R_{\text {Raman/Fluo }}$ rather suggests that $M_{\text {Tot }}$ is dominated by non-radiative enhancement. More experiments are needed to understand which phenomena are causing the secondary variations of $M_{\text {Tot }}$ at D3, D2, D1 powers but not at D0. This could be caused by an orientation effect of the molecule or by a multilayer effect for example.

Because of experimental limitations such as the fact that our CCD does not allow for integration times smaller than $0.027 \mathrm{~s}$ ( which would be necessary to access faster decays) and the fact that, on the other hand, we do not want to (or can not) carry out experiments for $10^{4}, 10^{5}$, $10^{6}$, (etc.) seconds, we will use low power excitation to access the fast initial photobleaching dynamics and high power excitation to access the slowest rates of the distribution. Our model of photobleaching of decay rates proportional to the incident power (see Eqs. 6.4 and 6.13), implies indeed that the photobleaching dynamics at, say, $10 S_{\text {inc }}$ should be 10 

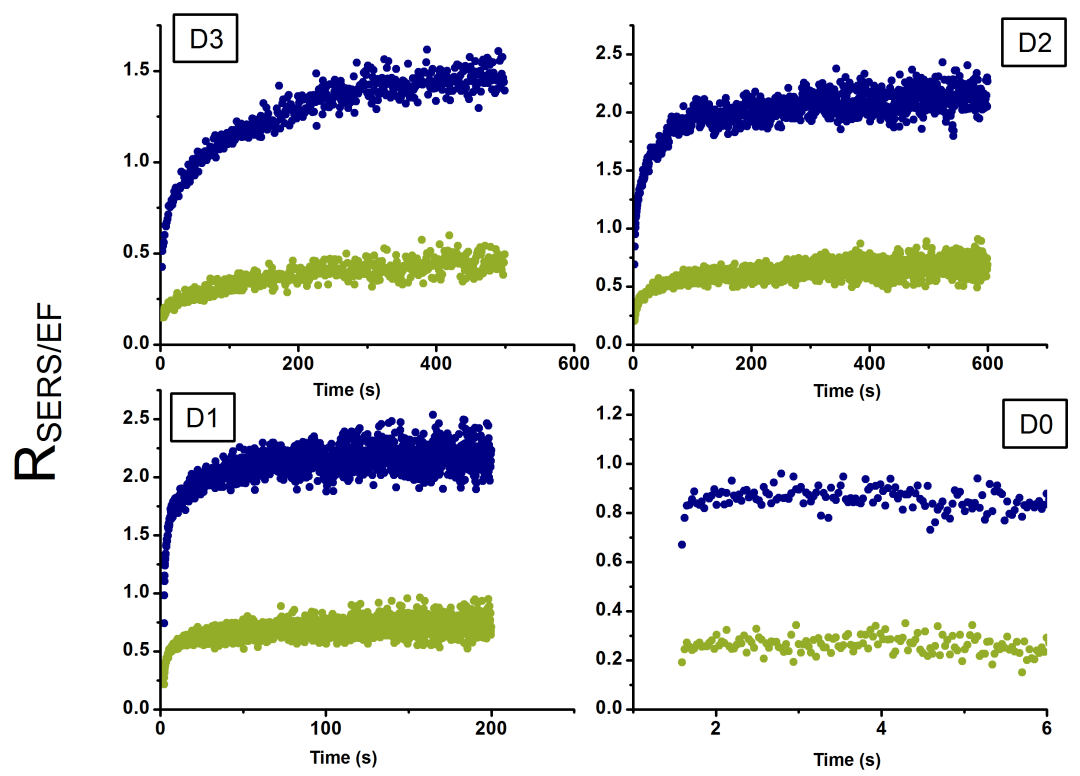

Figure 6.10: Dynamics of the ratio of SERS and SEF intensities for the $590 \mathrm{~cm}^{-1}$ (blue) and $1650 \mathrm{~cm}^{-1}$ (green) modes for the four powers D3-D0. The integration times for each power are given in Table 6.3.

times faster than the dynamics at $S_{\text {inc }}$, etc... In other words, taking into account the real power factors between the OD filters given in Table 6.2, the four intensities $I_{0}, I_{1}, I_{2}$ and $I_{3}$ defined by:

$$
\begin{aligned}
& I_{0}(t, \mathrm{D} 0)=I(t, \mathrm{D} 0) \\
& I_{1}(t, \mathrm{D} 0)=10.14 \times I(10.14 t, \mathrm{D} 1) \\
& I_{2}(t, \mathrm{D} 0)=105.23 \times I(105.23 t, \mathrm{D} 2) \\
& I_{3}(t, \mathrm{D} 0)=628.13 \times I(628.13 t, \mathrm{D} 3)
\end{aligned}
$$

should be equal, the intensities being all expressed in number of photons (or counts) per second. Figure 6.11 presents the 4 intensities of Eq. 6.18 on the same graph. While $I_{3}, I_{2}$ superimpose well, we can see that for higher incident powers $\left(I_{1}\right.$ and especially $\left.I(t, \mathrm{D} 0)\right)$ the model starts to fail (the intensity at D0 does not satisfy Eq. 6.18); the step down which is observed at D0 suggests that there is another effect which is not taken 
into account by our simple two-level model. The existence of a threshold in the incident power above which all molecules are instantaneously destroyed would explain such a shift. More experiments are needed in order to elucidate such a phenomenon.

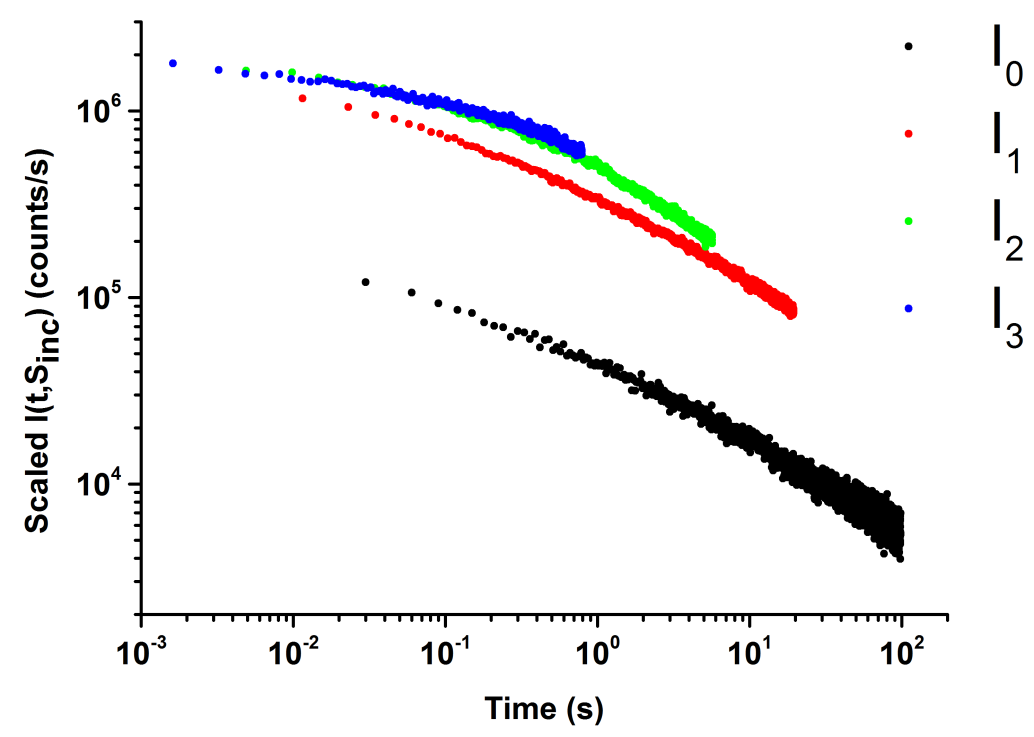

Figure 6.11: Comparison between $I(t, \mathrm{D} 0), I_{1}, I_{2}$ and $I_{3}$.

As a concluding remark, in analogy with the expression of the intensity decay rate of the bare molecule (Eq.6.4), we can define a phenomenological instantaneous decay rate $\Gamma(t)$ for the decay of the total SERS intensity:

$$
\text { If } \begin{aligned}
I_{\mathrm{SERS}} & =I_{0} e^{-\Gamma(t) t}, \quad \text { then } \ln \left(I / I_{0}\right)=-\Gamma(t) t \\
\Gamma(t) & =\frac{-\mathrm{d}(\ln I(t))}{\mathrm{d} t}
\end{aligned}
$$

Figure 6.12 shows a plot of $\Gamma(t)$ for all four powers. The measurements were done at different points on the sample for different powers. The times and intensities were all scaled with respect to D0, 1s integration time. We can see that the system presents photobleaching rates spanning 3-4 orders of magnitude; this constitutes a very peculiar photobleaching behavior and it is the first time it has been observed. 


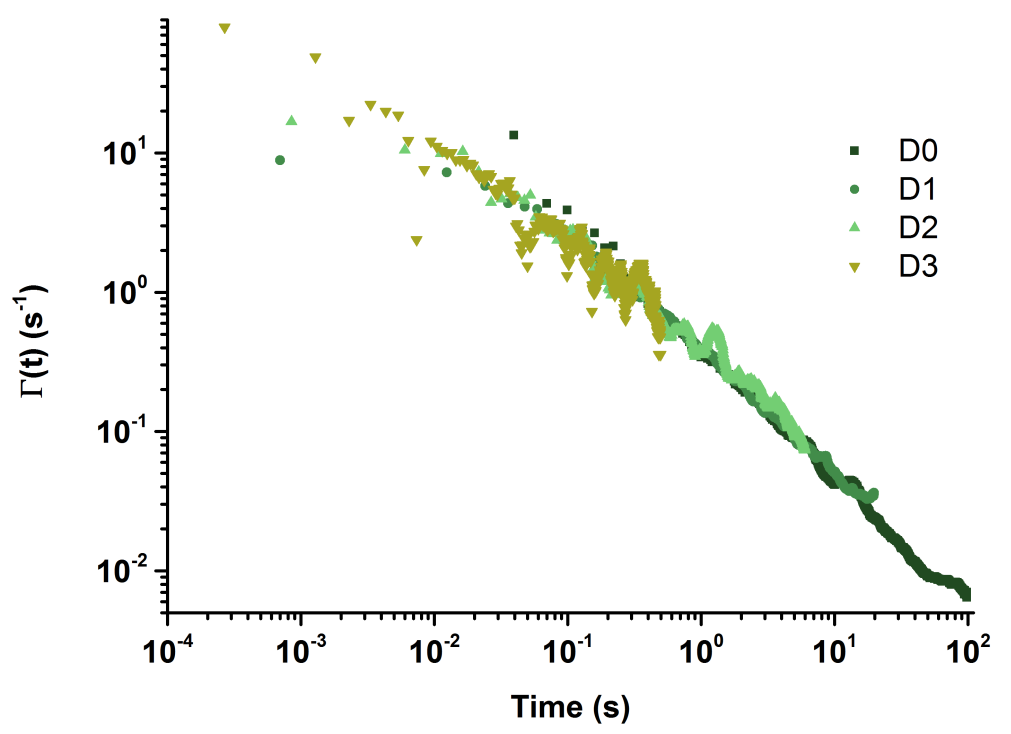

Figure 6.12: Instantaneous intensity decay rates $\Gamma(t)$. The times and intensities were scaled with respect to the times and intensities at D0.

\subsection{Conclusions and future work}

In conclusion, our simple two-level model and formalism for the photobleaching modified in the presence of a metallic substrate seems to be satisfactory for low powers (D3, D2 and D1 to some extent) excitations; i.e. the photobleaching rate $\Gamma_{\mathrm{PB}}$ of a molecule near to a metallic surface is proportional to the local field enhancement factor $M_{\mathrm{loc}}$ and the incident power. From there, it means there is an integral relationship between the time evolution of the total SERS (or SEF) intensity and the enhancement factor distribution $f\left(M_{\mathrm{loc}}\right)$ (Eq. 6.13). So, in principle, it should be possible to convert the intensity profile we get experimentally to a decay rate/enhancement factor distribution. However some numerical pitfalls are to be expected due to the integral form of the intensity.

Our model seems however to fail at describing the phenomena occurring at D0, maximum power $\left(1 \mathrm{~mW} \mathrm{\mu m}^{-2}\right)$. There seems to be some kind of threshold effect that our simple model does not take into account. This could be due to the heating of the gold nanodots or there could be a maximum local field intensity that a molecule at a hot spot could 
sustain without breaking. Further experiments will be needed in order to address this issue. 


\section{Conclusion and outlook}

The work presented in this thesis shows how SM-SERS can be a powerful tool to access subtle spectroscopic phenomena of molecules and in the last chapter paves the road to the understanding of photostability of probes under SERS conditions.

In Chapter 4, we see that SM-SERS spectra contain information about the homogeneous broadening of the vibrational modes of molecules which is lost in the average SERS spectrum. Due to the different interactions with the metallic substrate and the environment each molecule participating in the signal experiences, the central frequencies of each fingerprint mode "wanders" and is detected at slightly different positions. This results in a general broadening of the detected peaks, which can be much larger than the intrinsic homogeneous broadening. In the case of the fingerprint mode of the widely used dye Nile blue, the effect of these frequency variations is dramatic. It also provides an argument for the detection of single molecules; indeed if some events are much sharper in width than the average spectrum, they have to be single molecule events. Being able to measure the intrinsic broadening of the $590 \mathrm{~cm}^{-1}$ mode of individual Nile blue molecules by SERS has enabled us to unravel the strong temperature dependence of the width of single molecule events, temperature dependence which was absent on the overall average spectrum, hence completely masked by ensemble-averaging. Our temperature dependent measurements were consistent with the vibrational energy exchange theory of the broadening and suggest that the $590 \mathrm{~cm}^{-1}$ mode of Nile blue likely relaxes its population or phase through the excitation of vibrations in the silver substrate.

From here, future work could consist in the study of the homogeneous 
broadening of other molecules of interest and of other modes as it can ultimately be linked to the interactions of the mode with the metallic surface or with other intramolecular modes. There are also some outstanding issues: what is the influence of the electronic resonance on the homogeneous broadening, if any? Are there situations in which the homogeneous broadening of modes in the anti-Stokes part of the spectrum is different from the Stokes one?

In Chapter 5, we prove that the detection of the iconic $\mathrm{C}_{60}$ at the single molecule level is feasible by SERS. Our method can be seen as an extension of the bi-analyte method on isotopically edited partners as it relies on the dramatic effects that isotopic substitution of one or more ${ }^{12} \mathrm{C}$ by ${ }^{13} \mathrm{C}$ has on the Raman spectrum of $\mathrm{C}_{60}$. The frequency shift of the Raman active mode $A_{g}(2)$ of $\mathrm{C}_{60}$ induced by isotopic substitution is substantial and proportional to the number of ${ }^{12} \mathrm{C}$ atoms which have been replaced. Hence, in the case of the sample of $\mathrm{C}_{60}$ we studied whose isotopic content has been enriched, the resulting frequency shifts broaden dramatically the width of the $A_{g}(2)$ mode on the average Raman spectrum, in a similar manner to the inhomogeneous broadening described in Chapter 4. Single molecule events thus have a distribution of frequencies for this mode spanning a large spectral window, are all much sharper than the broad average and ultimately give us the ability to resolve different isotopic versions of $\mathrm{C}_{60}$, one by one, at the individual molecule level.

Generally, in SERS, homogeneity is very often obtained at the expense of electromagnetic SERS gain. Most of the SM-SERS studies rely on the statistical likelihood individual molecules will find the places of highest enhancement in the sample, hence rely on the "disorder" of systems such as colloids. Many spectra in the experiments of Chapter 4 were events sharper than the average but still much larger than the "threshold" width we found at each temperature. Some of these larger events may have been single molecule events but no statistical argument has enabled us to label them as such. In that case, these larger homogeneous widths could be interesting to study, but some more control over the SERS substrates is needed. 
Once more control of the substrate is achieved in the future, if SMSERS is to be widely used, one critical aspect will be that of the stability of molecules. Photobleaching is the main shortcoming of fluorescence but it has also a major bearing on SERS experiments on resonant probes as it affects the apparent SERS cross-section. In Chapter 6, we applied a simple two-level model of photobleaching together with the formalism for the different kinds of enhancement in SERS and SEF that have been developed by our group to the photostability of Nile blue under SERS conditions. For the aim of the study, Nile blue was deposited onto arrays of gold nanodots which constitutes a more homogeneous system than the SERS substrates we used in the two previous chapters. By analyzing the dynamics of the decay in the SERS intensity, we found that, in the limit of low incident power density, our model was satisfactory. The surfacemodified photobleaching rates of the different molecules contributing to the signal are proportional to the incident power and to the local field enhancement factor these molecules experience. These rates span four orders of magnitude and their distribution should reflect the distribution of enhancement factors on the surface.

Single molecule SERS is still a very young technique and the field has already grown enormously since 1997 and the heated debates of the last 10 years. It will soon reach a more established stage and be used to unveil phenomena that were out of our reach with measurements of ensembleaveraged signals. Understanding the spectroscopic nature and behavior of molecules adsorbed onto metallic surfaces is important to SERS as it can lead to a better understanding of the mechanisms underlying it but is also of tremendous value to surface sciences in general. 


\section{Bibliography}

[1] E. C. Le Ru and P. G. Etchegoin. Principles of surface enhanced Raman spectroscopy and related plasmonic effects. Elsevier, Amsterdam, 2009.

[2] D. A. Long. The Raman effect: a unified treatment of the theory of Raman scattering by molecules. Wiley \& Sons, 2002.

[3] B. Valeur and M. N. Berberan-Santos. Molecular fluorescence: principles and applications. John Wiley \& Sons, 2013.

[4] C. V. Raman and K. S. Krishnan. A new type of secondary radiation. Nature, 121(3048):501-502, 1928.

[5] H. W. Schrötter and H. W. Klöckner. Raman scattering cross sections in gases and liquids. In Raman spectroscopy of gases and liquids, pages 123-166. Springer, 1979.

[6] B. Auguié, A. Reigue, E. C. Le Ru, and P. G. Etchegoin. Tiny peaks vs mega backgrounds: a general spectroscopic method with applications in resonant Raman scattering and atmospheric absorptions. Analytical Chemistry, 84(18):7938-7945, 2012.

[7] A. Reigue, B. Auguié, P. G. Etchegoin, and E. C. Le Ru. CW measurements of resonance Raman profiles, line-widths, and crosssections of fluorescent dyes: application to Nile blue A in water and ethanol. Journal of Raman Spectroscopy, 44(4):573-581, 2013.

[8] M. Fleischmann, P. J. Hendra, and A. J. McQuillan. Raman spectra of pyridine adsorbed at a silver electrode. Chemical Physics Letters, 26(2):163-166, 1974. 
[9] D. L. Jeanmaire and R. P. Van Duyne. Surface Raman spectroelectrochemistry: Part I. Heterocyclic, aromatic, and aliphatic amines adsorbed on the anodized silver electrode. Journal of Electroanalytical Chemistry and Interfacial Electrochemistry, 84(1):1-20, 1977.

[10] M. G. Albrecht and J. A. Creighton. Anomalously intense Raman spectra of pyridine at a silver electrode. Journal of the American Chemical Society, 99(15):5215-5217, 1977.

[11] E. C. Le Ru and P. G. Etchegoin. Rigorous justification of the $|E|^{4}$ enhancement factor in surface enhanced Raman spectroscopy. Chemical Physics Letters, 423(1-3):63-66, 2006.

[12] E. C. Le Ru and P. G. Etchegoin. Quantifying SERS enhancements. MRS bulletin, 38(08):631-640, 2013.

[13] E. C. Le Ru, P. G. Etchegoin, and M. Meyer. Enhancement factor distribution around a single surface-enhanced Raman scattering hot spot and its relation to single molecule detection. The Journal of Chemical Physics, 125(20):204701, 2006.

[14] E. C. Le Ru and P. G. Etchegoin. Phenomenological local field enhancement factor distributions around electromagnetic hot spots. The Journal of Chemical Physics, 130(18):181101, 2009.

[15] R. Aroca. Surface-enhanced vibrational spectroscopy. Wiley, 2006.

[16] J. A. Dieringer, K. L. Wustholz, D. J. Masiello, J. P. Camden, S. L. Kleinman, G. C. Schatz, and R. P. Van Duyne. Surface-enhanced Raman excitation spectroscopy of a single rhodamine $6 \mathrm{G}$ molecule. Journal of the American Chemical Society, 131(2):849-854, 2009. PMID: 19140802.

[17] S. Nie and S. R. Emory. Probing single molecules and single nanoparticles by surface-enhanced Raman scattering. Science, 275(5303):1102-1106, 1997. 
[18] K. Kneipp, Y. Wang, H. Kneipp, L. T. Perelman, I. Itzkan, R. R. Dasari, and M. S. Feld. Single molecule detection using surfaceenhanced Raman scattering (SERS). Physical Review Letters, 78(9):1667, 1997.

[19] E. C. Le Ru, E. Blackie, M. Meyer, and P. G. Etchegoin. Surface enhanced Raman scattering enhancement factors: a comprehensive study. The Journal of Physical Chemistry C, 111(37):13794-13803, 2007.

[20] M. Moskovits. Persistent misconceptions regarding SERS. Physical Chemistry Chemical Physics, 15(15):5301-5311, 2013.

[21] E. C. Le Ru, M. Meyer, and P. G. Etchegoin. Proof of singlemolecule sensitivity in surface enhanced Raman scattering (SERS) by means of a two-analyte technique. The Journal of Physical Chemistry B, 110(4):1944-1948, 2006.

[22] J. A. Dieringer, R. B. Lettan, K. A. Scheidt, and R. P. Van Duyne. A frequency domain existence proof of single-molecule surfaceenhanced Raman spectroscopy. Journal of the American Chemical Society, 129(51):16249-16256, 2007.

[23] P. G. Etchegoin, M. Meyer, E. Blackie, and E. C. Le Ru. Statistics of single-molecule surface enhanced Raman scattering signals: fluctuation analysis with multiple analyte techniques. Analytical Chemistry, 79(21):8411-8415, 2007.

[24] Y. Sawai, B. Takimoto, H. Nabika, K. Ajito, and K. Murakoshi. Observation of a small number of molecules at a metal nanogap arrayed on a solid surface using surface-enhanced Raman scattering. Journal of the American Chemical Society, 129(6):1658-1662, 2007.

[25] E. Blackie, E. C. Le Ru, M. Meyer, M. Timmer, B. Burkett, P. Northcote, and P. G. Etchegoin. Bi-analyte SERS with isotopically edited dyes. Physical Chemistry Chemical Physics, 10(28):4147-4153, 2008. 
[26] D. Zhang, Y. Xie, S. K. Deb, V. J. Davison, and D. Ben-Amotz. Isotope edited internal standard method for quantitative surfaceenhanced Raman spectroscopy. Analytical Chemistry, 77(11):35633569, 2005.

[27] C. Galloway, P. Etchegoin, and E. Le Ru. Ultrafast nonradiative decay rates on metallic surfaces by comparing surface-enhanced Raman and fluorescence signals of single molecules. Physical Review Letters, 103(6):063003, 2009.

[28] W. H. Park and Z. H. Kim. Charge transfer enhancement in the SERS of a single molecule. Nano Letters, 10(10):4040-4048, 2010.

[29] P. G. Etchegoin, P. D. Lacharmoise, and E. C. Le Ru. Influence of photostability on single-molecule surface enhanced Raman scattering enhancement factors. Analytical Chemistry, 81(2):682-688, 2009.

[30] E. J. Blackie, E. C. Le Ru, and P. G. Etchegoin. Single-molecule surface-enhanced Raman spectroscopy of nonresonant molecules. Journal of the American Chemical Society, 131(40):14466-14472, 2009 .

[31] Z. L. Zhang, Y. F. Yin, J. W. Jiang, and Y. J. Mo. Single molecule detection of 4-dimethylaminoazobenzene by surface-enhanced Raman spectroscopy. Journal of Molecular Structure, 920(1):297-300, 2009 .

[32] D. R. Ward, N. J. Halas, J. W. Ciszek, J. M. Tour, Y. Wu, P. Nordlander, and D. Natelson. Simultaneous measurements of electronic conduction and Raman response in molecular junctions. Nano Letters, 8(3):919-924, 2008.

[33] D. R. Ward, D. A. Corley, J. M. Tour, and D. Natelson. Vibrational and electronic heating in nanoscale junctions. Nature Nanotechnology, 6(1):33-38, 2011. 
[34] E. Cortés, P. G. Etchegoin, E. C. Le Ru, A. Fainstein, M. E. Vela, and R. C. Salvarezza. Monitoring the electrochemistry of single molecules by surface-enhanced Raman spectroscopy. Journal of the American Chemical Society, 132(51):18034-18037, 2010.

[35] E. Cortés, P. G. Etchegoin, E. C. Le Ru, A. Fainstein, M. E. Vela, and R. C. Salvarezza. Strong correlation between molecular configurations and charge-transfer processes probed at the singlemolecule level by surface-enhanced Raman scattering. Journal of the American Chemical Society, 135(7):2809-2815, 2013.

[36] E. C. Le Ru and P. G. Etchegoin. Phenomenological local field enhancement factor distributions around electromagnetic hot spots. The Journal of Chemical Physics, 130(18):181101, 2009.

[37] S. A. Meyer, E. C. Le Ru, and P. G. Etchegoin. Combining surface plasmon resonance (SPR) spectroscopy with surface-enhanced Raman scattering (SERS). Analytical Chemistry, 83(6):2337-2344, 2011.

[38] S. A. Meyer, B. Auguié, E. C. Le Ru, and P. G. Etchegoin. Combined SPR and SERS microscopy in the Kretschmann configuration. The Journal of Physical Chemistry A, 116(3):1000-1007, 2012 .

[39] H. Xu, J. Aizpurua, M. Käll, and P. Apell. Electromagnetic contributions to single-molecule sensitivity in surface-enhanced Raman scattering. Physical Review E, 62(3):4318, 2000.

[40] A. Weiss and G. Haran. Time-dependent single-molecule Raman scattering as a probe of surface dynamics. The Journal of Physical Chemistry B, 105(49):12348-12354, 2001.

[41] E. C. Le Ru and P. G. Etchegoin. Single-molecule surface-enhanced Raman spectroscopy. Annual Review of Physical Chemistry, 63:6587, 2012. 
[42] H. Xu, E. J. Bjerneld, M. Käll, and L. Börjesson. Spectroscopy of single hemoglobin molecules by surface enhanced Raman scattering. Physical Review Letters, 83(21):4357, 1999.

[43] P. C. Lee and D. Meisel. Adsorption and surface-enhanced Raman of dyes on silver and gold sols. The Journal of Physical Chemistry, 86(17):3391-3395, 1982.

[44] C. H. Munro, W. E. Smith, M. Garner, J. W. P. C. Clarkson, and P. C. White. Characterization of the surface of a citrate-reduced colloid optimized for use as a substrate for surface-enhanced resonance Raman scattering. Langmuir, 11(10):3712-3720, 1995.

[45] N. Grillet, D. Manchon, E. Cottancin, F. Bertorelle, C. Bonnet, M. Broyer, J. Lerme, and M. Pellarin. Photo-oxidation of individual silver nanoparticles: a real-time tracking of optical and morphological changes. The Journal of Physical Chemistry C, 117(5):2274$2282,2013$.

[46] M. Meyer, E. C. Le Ru, and P. G. Etchegoin. Self-limiting aggregation leads to long-lived metastable clusters in colloidal solutions. The Journal of Physical Chemistry B, 110(12):6040-6047, 2006.

[47] S. E. J. Bell and N. M. S. Sirimuthu. Surface-enhanced Raman spectroscopy as a probe of competitive binding by anions to citratereduced silver colloids. The Journal of Physical Chemistry A, 109(33):7405-7410, 2005.

[48] J. A. Creighton, C. G. Blatchford, and M. G. Albrecht. Plasma resonance enhancement of Raman scattering by pyridine adsorbed on silver or gold sol particles of size comparable to the excitation wavelength. Journal of the Chemical Society, Faraday Transactions 2, 75:790-798, 1979.

[49] N. Leopold and B. Lendl. A new method for fast preparation of highly surface-enhanced Raman scattering (SERS) active silver colloids at room temperature by reduction of silver nitrate with hy- 
droxylamine hydrochloride. The Journal of Physical Chemistry B, 107(24):5723-5727, 2003.

[50] V. M. Shalaev. Electromagnetic properties of small-particle composites. Physics Reports, 272(2):61-137, 1996.

[51] P. G. Etchegoin and E. C. Le Ru. Resolving single molecules in surface-enhanced Raman scattering within the inhomogeneous broadening of Raman peaks. Analytical Chemistry, 82(7):28882892, 2010.

[52] C. M. Galloway, E. C. Le Ru, and P. G. Etchegoin. Singlemolecule vibrational pumping in SERS. Physical Chemistry Chemical Physics, 11(34):7372-7380, 2009.

[53] E. C. Le Ru, S. A. Meyer, C. Artur, P. G. Etchegoin, J. Grand, P. Lang, and F. Maurel. Experimental demonstration of surface selection rules for SERS on flat metallic surfaces. Chemical Communications, 47:3903-3905, 2011.

[54] P. G. Etchegoin, E. C. Le Ru, and M. Meyer. Evidence of natural isotopic distribution from single-molecule SERS. Journal of the American Chemical Society, 131(7):2713-2716, 2009.

[55] K. Sekiguchi, S. Yamaguchi, and T. Tahara. Formation and dissociation of rhodamine 800 dimers in water: steady-state and ultrafast spectroscopic study. The Journal of Physical Chemistry A, 110(8):2601-2606, 2006.

[56] G. K. Wertheim, M. A. Butler, K. W. West, and D. N. E. Buchanan. Determination of the Gaussian and Lorentzian content of experimental line shapes. Review of Scientific Instruments, 45(11):1369-1371, 2003.

[57] L. S. Lasdon, A. D. Waren, A. Jain, and M. Ratner. Design and testing of a generalized reduced gradient code for nonlinear programming. ACM Transactions on Mathematical Software (TOMS), 4(1):34-50, 1978. 
[58] W. Demtröder. Laser Spectroscopy, volume 1. Springer, Berlin, 2008.

[59] O. Madelung. Introduction to Solid-State Theory, volume 2. Springer, 1996.

[60] A. L. Harris, L. Rothberg, L. H. Dubois, N. J. Levinos, and L. Dhar. Molecular vibrational energy relaxation at a metal surface: methyl thiolate on $\mathrm{Ag}(111)$. Physical Review Letters, 64(17):2086-2089, 1990.

[61] A. Harris, L. Rothberg, L. Dhar, N. Levinos, and L. Dubois. Vibrational energy relaxation of a polyatomic adsorbate on a metal surface: methyl thiolate $\left(\mathrm{CH}_{3} \mathrm{~S}\right)$ on $\mathrm{Ag}$ (111). The Journal of Chemical Physics, 94(4):2438-2448, 1991.

[62] P. Avouris and B. N. J. Persson. Excited states at metal surfaces and their non-radiative relaxation. The Journal of Physical Chemistry, 88(5):837-848, 1984.

[63] B. N. J. Persson and R. Ryberg. Vibrational phase relaxation at surfaces: CO on Ni (111). Physical Review Letters, 54:2119-2122, 1985 .

[64] R. Kroon, R. Sprik, and A. Lagendijk. Picosecond vibrational relaxation modulated by critical slowing down in ferroelectric $\mathrm{K}_{4} \mathrm{Fe}(\mathrm{CN})_{6}\left(\mathrm{H}_{2} \mathrm{O}\right)_{3}$. Physical Review B, 42(5):2785, 1990.

[65] J. W. Gadzuk and A. C. Luntz. On vibrational lineshapes of adsorbed molecules. Surface Science, 144:429-450, 1984.

[66] R. M. Shelby, C. B. Harris, and P. A. Cornelius. The origin of vibrational dephasing of polyatomic molecules in condensed phases. The Journal of Chemical Physics, 70(1):34-41, 1979.

[67] H. Arnolds and M. Bonn. Ultrafast surface vibrational dynamics. Surface Science Reports, 65(2):45-66, 2010. 
[68] V. Kozich, J. Dreyer, S. Ashihara, W. Werncke, and T. Elsaesser. Mode-selective O-H stretching relaxation in a hydrogen bond studied by ultrafast vibrational spectroscopy. The Journal of Chemical Physics, 125(7):074504, 2006.

[69] S. Mukamel. Principles of nonlinear optical spectroscopy, volume 29. Oxford University Press New York, 1995.

[70] G. Deinzer, M. Schmitt, A. P. Mayer, and D. Strauch. Intrinsic lifetimes and anharmonic frequency shifts of long-wavelength optical phonons in polar crystals. Physical Review B, 69(1):014304, 2004

[71] J. Serrano, M. Cardona, T. M. Ritter, B. A. Weinstein, A. Rubio, and C. T. Lin. Pressure and temperature dependence of the Raman phonons in isotopic $\gamma$-CuI. Physical Review B, 66(24):245202, 2002.

[72] M. J. Clouter, H. Kiefte, and R. K. Jain. Pure vibrational Raman spectra of simple liquids: $\mathrm{O}_{2}, \mathrm{~N}_{2}, \mathrm{CO}, \mathrm{CH}_{4}, \mathrm{CF}_{4}$. The Journal of Chemical Physics, 73(2):673-682, 2008.

[73] S. Marks, P. A. Cornelius, and C. B. Harris. A critical test of vibrational dephasing theories in solids using spontaneous Raman scattering in isotopically mixed crystals. The Journal of Chemical Physics, 73(7):3069-3081, 1980.

[74] D. Hsu and J. L. Skinner. Nonperturbative theory of temperaturedependent optical dephasing in crystals. I. Acoustic or optical phonons. The Journal of Chemical Physics, 81(12):5471-5479, 1984.

[75] H. M. Sevian and J. L. Skinner. $T_{2}$ can be greater than $2 T_{1}$. The Journal of Chemical Physics, 91(3):1775-1782, 1989.

[76] P. W. Anderson. A mathematical model for the narrowing of spectral lines by exchange or motion. Journal of the Physical Society of Japan, 9(3):316-339, 1954. 
[77] R. Kubo and K. Tomita. A general theory of magnetic resonance absorption. Journal of the Physical Society of Japan, 9(6):888-919, 1954.

[78] S. F. Fischer and A. Laubereau. Dephasing processes of molecular vibrations in liquids. Chemical Physics Letters, 35(1):6-12, 1975.

[79] D. J. Diestler. Vibrational phase and energy relaxation in the broadening of Raman spectral lines in dense media. Chemical Physics Letters, 39(1):39-44, 1976.

[80] C. B. Harris, R. M. Shelby, and P. A. Cornelius. Effects of energy exchange on vibrational dephasing times in Raman scattering. Physical Review Letters, 38(24):1415, 1977.

[81] H. W. Kroto, J. R. Heath, S. C. O'Brien, R. F. Curl, and R. E. Smalley. $\mathrm{C}_{60}$ : buckminsterfullerene. Nature, 318:162-163, 1985.

[82] S. Iijima. Helical microtubules of graphitic carbon. Nature, 354(6348):56-58, 1991.

[83] M. Campoy-Quiles, T. Ferenczi, T. Agostinelli, P. G. Etchegoin, Y. Kim, T. D. Anthopoulos, P. N. Stavrinou, D. D. C. Bradley, and J. Nelson. Morphology evolution via self-organization and lateral and vertical diffusion in polymer: fullerene solar cell blends. Nature Materials, 7(2):158-164, 2008.

[84] M. S. Dresselhaus, G. Dresselhaus, and P. C. Eklund. Sciences of Fullerenes and Carbon Nanotubes: Their properties and Applications. Elsevier, New York, 1996.

[85] D. E. H. Jones. New Scientist, 32(245):1, 1966.

[86] H. Kroto. Space, stars, $\mathrm{C}_{60}$, and soot. Science, 242(4882):1139, 1988.

[87] E. Osawa. Superaromaticity. Kagaku, 25:854-863, 1970. 
[88] R. D. Johnson, G. Meijer, and D. S. Bethune. $\mathrm{C}_{60}$ has icosahedral symmetry. Journal of the American Chemical Society, 112(24):8983-8984, 1990.

[89] P. Zhou, K.-A. Wang, Y. Wang, P. C. Eklund, M. S. Dresselhaus, G. Dresselhaus, and R. A. Jishi. Raman scattering in $\mathrm{C}_{60}$ and alkali-metal-saturated $\mathrm{C}_{60}$. Physical Review B, 46:2595-2605, 1992.

[90] P. C. Eklund, P. Zhou, K. A. Wang, G. Dresselhaus, and M. S. Dresselhaus. Optical phonon modes in solid and doped $\mathrm{C}_{60}$. Journal of Physics and Chemistry of Solids, 53:1391, 1992.

[91] K. A. Wang, Y. Wang, P. Zhou, J. M. Holden, S. L. Ren, G. T. Hager, H. F. Ni, P. C. Eklund, G. Dresselhaus, and M. S. Dresselhaus. Raman scattering in $\mathrm{C}_{60}$ and alkali-metal-doped $\mathrm{C}_{60}$ films. Physical Review B, 45:1955-1958, 1992.

[92] S. J. Duclos, R. C. Haddon, S. Glarum, A. F. Hebard, and K. B. Lyons. Raman studies of alkali-metal doped $\mathrm{A}_{\mathrm{x}} \mathrm{C}_{60}$ films $(\mathrm{A}=\mathrm{Na}$, $\mathrm{K}, \mathrm{Rb}$, and $\mathrm{Cs} ; \mathrm{x}=0.3$ and 6). Science, 254(5038), 1991.

[93] D. S. Bethune, G. Meijer, W. C. Tang, and H. J. Rosen. The vibrational Raman spectra of purified solid films of $\mathrm{C}_{60}$ and $\mathrm{C}_{70}$. Chemical Physics Letters, 174:219-222, 1990.

[94] P. Zhou, A. M. Rao, K. A. Wang, J. D. Robertson, C. Eloi, M. S. Meier, S. L. Ren, X. X. Bi, P. C. Eklund, and M. S. Dresselhaus. Photo-assisted structural transition and oxygen diffusion in solid $\mathrm{C}_{60}$ films. Applied Physics Letters, 60:2871-2873, 1992.

[95] D. W. Snoke and M. Cardona. A bond polarizability model for the $\mathrm{C}_{60}$ Raman spectrum. Solid State Communications, 87(2):121-126, 1993.

[96] S. Guha, J. Menendez, J. B. Page, and G. B. Adams. Empirical bond polarizability model for fullerenes. Physical Review B, 53(19):13106, 1996. 
[97] S. Guha, J. Menendez, J. B. Page, G. B. Adams, G. S. Spencer, J. P. Lehman, P. Giannozzi, and S. Baroni. Isotopically resolved Raman spectra of $\mathrm{C}_{60}$. Physical Review Letters, 72(21):3359-3362, 1994.

[98] J. D. Lorentzen, S. Guha, J. Menendez, P. Giannozzi, and S. Baroni. Raman cross section for the pentagonal-pinch mode in buckminsterfullerene $\mathrm{C}_{60}$. Chemical Physics Letters, 270(1):129 - 134, 1997.

[99] A. M. Rao, P. Zhou, K. A. Wang, G. T. Hager, J. M. Holden, Y. Wang, W. T. Lee, X. X. Bi, P. C. Ecklund, and D. S. Cornett. Photoinduced polymerization of solid $\mathrm{C}_{60}$ films. Science, 259:955957, 1993.

[100] P. J. Horoyski and M. L. W. Thewalt. Optically detected librons and phonons in crystalline $\mathrm{C}_{60}$. Physical Review B, 48(15):11446, 1993.

[101] M. E. Kozlov and K. Yakushi. Optical properties of high-pressure phases of $\mathrm{C}_{60}$ fullerene. Journal of Physics: Condensed Matter, 7(15):L209, 1999.

[102] K. Sinha, J. Menendez, R. C. Hanson, G. B. Adams, J. B. Page, O. F. Sankey, L. D. Lamb, and D. R. Huffman. Evidence for solidstate effects in the electronic structure of $\mathrm{C}_{60}$ films: a resonance Raman study. Chemical Physics Letters, 186(2):287-290, 1991.

[103] P. J. Horoyski, M. L. W. Thewalt, and T. R. Anthony. Raman fine structure in crystalline $\mathrm{C}_{60}$ : the effects of merohedral disorder, isotopic substitution, and crystal field. Physical Review Letters, 74(1):194-197, 1995.

[104] C. S. Yannoni, P. P. Bernier, D. S. Bethune, G. Meijer, and J. R. Salem. NMR determination of the bond lengths in $\mathrm{C}_{60}$. Journal of the American Chemical Society, 113(8):3190-3192, 1991. 
[105] W. I. F. David, R. M. Ibberson, J. C. Matthewman, K. Prassides, T. J. S. Dennis, J. P. Hare, H. W. Kroto, R. Taylor, and D. R. M. Walton. Crystal structure and bonding of ordered $\mathrm{C}_{60}$. Nature, 353(6340):147-149, 1991.

[106] R. D. Johnson, D. S. Bethune, and C. S. Yannoni. Fullerene structure and dynamics: a magnetic resonance potpourri. Accounts of Chemical Research, 25(3):169-175, 1992.

[107] M. S. Amer. Raman spectroscopy, fullerenes and nanotechnology. Royal Society of Chemistry, 2010.

[108] W. G. Harter and T. C. Reimer. Nuclear spin weights and gas phase spectral structure of ${ }^{12} \mathrm{C}_{60}$ and ${ }^{13} \mathrm{C}_{60}$ buckminsterfullerene. Chemical Physics Letters, 194(3):230-238, 1992.

[109] M. Cardona and G. Güntherodt. Vibrational spectroscopy of $\mathrm{C}_{60}$. In Light Scattering in Solids VIII, pages 1-26. Springer, 2000.

[110] J. Menéndez, J. B. Page, and S. Guha. The isotope effect on the Raman spectrum of molecular $\mathrm{C}_{60}$. Philosophical Magazine B, 70(3):651-659, 1994.

[111] D. Heymann. Solubility of fullerenes $\mathrm{C}_{60}$ and $\mathrm{C}_{70}$ in seven normal alcohols and their deduced solubility in water. Fullerene Science 8 Technology, 4(3):509-515, 1996.

[112] R. S. Ruoff, D. S. Tse, R. Malhotra, and D. C. Lorents. Solubility of fullerene $\mathrm{C}_{60}$ in a variety of solvents. The Journal of Physical Chemistry, 97(13):3379-3383, 1993.

[113] M. J. Frisch, G. W. Trucks, H. B. Schlegel, G. E. Scuseria, M. A. Robb, J. R. Cheeseman, G. Scalmani, V. Barone, B. Mennucci, G. A. Petersson, H. Nakatsuji, M. Caricato, X. Li, H. P. Hratchian, A. F. Izmaylov, J. Bloino, G. Zheng, J. L. Sonnenberg, M. Hada, M. Ehara, K. Toyota, R. Fukuda, J. Hasegawa, M. Ishida, T. Nakajima, Y. Honda, O. Kitao, H. Nakai, T. Vreven, J. A. Montgomery, 
Jr., J. E. Peralta, F. Ogliaro, M. Bearpark, J. J. Heyd, E. Brothers, K. N. Kudin, V. N. Staroverov, R. Kobayashi, J. Normand, K. Raghavachari, A. Rendell, J. C. Burant, S. S. Iyengar, J. Tomasi, M. Cossi, N. Rega, J. M. Millam, M. Klene, J. E. Knox, J. B. Cross, V. Bakken, C. Adamo, J. Jaramillo, R. Gomperts, R. E. Stratmann, O. Yazyev, A. J. Austin, R. Cammi, C. Pomelli, J. W. Ochterski, R. L. Martin, K. Morokuma, V. G. Zakrzewski, G. A. Voth, P. Salvador, J. J. Dannenberg, S. Dapprich, A. D. Daniels, Ö. Farkas, J. B. Foresman, J. V. Ortiz, J. Cioslowski, and D. J. Fox. Gaussian 09 Revision B.01. Gaussian Inc. Wallingford CT 2009 .

[114] F. Nogueira, A. Castro, and M. A. L. Marques. A tutorial on density functional theory. In A Primer in Density Functional Theory, pages 218-256. Springer, 2003.

[115] P. Giannozzi and S. Baroni. Vibrational and dielectric properties of $\mathrm{C}_{60}$ from density-functional perturbation theory. The Journal of Chemical Physics, 100(11):8537-8539, 1994.

[116] V. Schettino, M. Pagliai, L. Ciabini, and G. Cardini. The vibrational spectrum of fullerene $\mathrm{C}_{60}$. The Journal of Physical Chemistry A, 105(50):11192-11196, 2001.

[117] W. Kohn, A. D. Becke, and R. G. Parr. Density functional theory of electronic structure. The Journal of Physical Chemistry, 100(31):12974-12980, 1996.

[118] C. Lee, W. Yang, and R. G. Parr. Development of the Colle-Salvetti correlation-energy formula into a functional of the electron density. Physical Review B, 37:785-789, 1988.

[119] S. H. Vosko, L. l. Wilk, and M. Nusair. Accurate spin-dependent electron liquid correlation energies for local spin density calculations: a critical analysis. Canadian Journal of Physics, 58(8):1200$1211,1980$. 
[120] P. J. Stephens, F. J. Devlin, C. F. Chabalowski, and M. J. Frisch. $\mathrm{Ab}$ initio calculation of vibrational absorption and circular dichroism spectra using density functional force fields. The Journal of Physical Chemistry, 98(45):11623-11627, 1994.

[121] R. B. J. S. Krishnan, J. S. Binkley, R. Seeger, and J. A. Pople. Selfconsistent molecular orbital methods. XX. A basis set for correlated wave functions. The Journal of Chemical Physics, 72(1):650-654, 1980 .

[122] M. S. Dresselhaus, M. A. Pimenta, K. Kneipp, S. D. M. Brown, P. Corio, A. Marucci, and G. Dresselhaus. First and secondorder resonant Raman spectra of single-walled carbon nanotubes. In M. Thorpe, D. Tomnek, and R. Enbody, editors, Science and Application of Nanotubes, Fundamental Materials Research, pages 253-274. Springer US, 2002.

[123] K. L. Akers, L. M. Cousins, and M. Moskovits. Surface-enhanced vibrational Raman spectroscopy of $\mathrm{C}_{60}$ and $\mathrm{C}_{70}$ on rough silver surfaces. Chemical Physics Letters, 190(6):614-620, 1992.

[124] S. J. Chase, W. S. Bacsa, M. G. Mitch, L. J. Pilione, and J. S. Lannin. Surface-enhanced Raman scattering and photoemission of $\mathrm{C}_{60}$ on noble-metal surfaces. Physical Review B, 46(12):7873, 1992.

[125] M. Baibarac, L. Mihut, N. Preda, I. Baltog, J. Y. Mevellec, and S. Lefrant. Surface-enhanced Raman scattering studies on $\mathrm{C}_{60}$ fullerene self-assemblies. Carbon, 43(1):1-9, 2005.

[126] R. L. Garrell, T. M. Herne, C. A. Szafranski, F. Diederich, F. Ettl, and R. L. Whetten. Surface-enhanced raman spectroscopy of $\mathrm{C}_{60}$ on gold: evidence for symmetry reduction and perturbation of electronic structure in the adsorbed molecule. Journal of the American Chemical Society, 113(16):6302-6303, 1991.

[127] C. Artur, E. C. Le Ru, and P. G. Etchegoin. Temperature dependence of the homogeneous broadening of resonant Raman 
peaks measured by single-molecule surface-enhanced Raman spectroscopy. The Journal of Physical Chemistry Letters, 2(23):30023005, 2011.

[128] J. Spitzer, P. Etchegoin, M. Cardona, T. R. Anthony, and W. F. Banholzer. Isotopic-disorder induced Raman scattering in diamond. Solid state communications, 88(7):509-514, 1993.

[129] W. Babel, I. Endo, S. O. Enfors, A. Fiechter, M. Hoare, W. S. Hu, B. Mattiasson, J. Nielsen, H. Sahm, K. Schügerl, et al. Advances in Biochemical Engineering/Biotechnology. Tissue Engineering, 104, 2007.

[130] D. M. Soumpasis. Theoretical analysis of fluorescence photobleaching recovery experiments. Biophysical journal, 41(1):95-97, 1983.

[131] V. Kasche and L. Lindqvist. Reactions between the triplet state of fluorescein and oxygen. The Journal of Physical Chemistry, 68(4):817-823, 1964.

[132] Y. Usui, K. Itoh, and M. Koizumi. Switch-over of the mechanism of the primary processes in the photo-oxidation of xanthene dyes as revealed by the oxygen consumption experiments. Bulletin of the Chemical Society of Japan, 38(6):1015-1022, 1965.

[133] E. C. Le Ru, P. G. Etchegoin, J. Grand, N. Félidj, J. Aubard, and G. Lévi. Mechanisms of spectral profile modification in surfaceenhanced fluorescence. The Journal of Physical Chemistry C, 111(44):16076-16079, 2007.

[134] P. G. Etchegoin, E. C. Le Ru, R. C. Maher, and L. F. Cohen. Enhancement factor averaging and the photostability of probes in SERS vibrational pumping. Physical Chemistry Chemical Physics, 9(35):4923-4929, 2007.

[135] G. Sun and J. B. Khurgin. Origin of giant difference between fluorescence, resonance, and nonresonance Raman scattering enhancement by surface plasmons. Physical Review A, 85(6):063410, 2012. 
[136] L. A. Deschenes and D. A. Vanden Bout. Single molecule photobleaching: increasing photon yield and survival time through suppression of two-step photolysis. Chemical Physics Letters, 365(5):387-395, 2002.

[137] E. J. Bjerneld, F. Svedberg, P. Johansson, and M. Käll. Direct observation of heterogeneous photochemistry on aggregated Ag nanocrystals using Raman spectroscopy: The case of photoinduced degradation of aromatic amino acids. The Journal of Physical Chemistry A, 108(19):4187-4193, 2004.

[138] B. Pettinger, B. Ren, G. Picardi, R. Schuster, and G. Ertl. Tipenhanced Raman spectroscopy (TERS) of malachite green isothiocyanate at $\mathrm{Au}$ (111): bleaching behavior under the influence of high electromagnetic fields. Journal of Raman Spectroscopy, 36(67):541-550, 2005.

[139] N. Félidj, J. Aubard, G. Lévi, J. R. Krenn, M. Salerno, G. Schider, B. Lamprecht, A. Leitner, and F. R. Aussenegg. Controlling the optical response of regular arrays of gold particles for surfaceenhanced Raman scattering. Physical Review B, 65(7):075419, 2002

[140] N. Félidj, J. Aubard, G. Lévi, J. R. Krenn, A. Hohenau, G. Schider, A. Leitner, and F. R. Aussenegg. Optimized surface-enhanced Raman scattering on gold nanoparticle arrays. Applied Physics Letters, 82(18):3095-3097, 2003.

[141] D. A. Weitz, S. Garoff, and T. J. Gramila. Excitation spectra of surface-enhanced Raman scattering on silver-island films. Optics letters, 7(4):168-170, 1982. 